\author{
Aus der \\ Klinik und Poliklinik für Augenheilkunde \\ der Medizinischen Fakultät \\ der Otto-von-Guericke Universität Magdeburg
}

\title{
Untersuchung des Farbsehens mittels Pigmentfarbtafeln und Tablet-PC
}

\author{
Dissertation \\ zur Erlangung des akademischen Grades \\ Dr. med. \\ (doctor medicinae) \\ an der Medizinischen Fakultät \\ der Otto-von-Guericke Universität Magdeburg
}

vorgelegt von Panagiota Tsimpri

aus Berlin

Magdeburg 2015 


\section{Bibliographische Beschreibung}

Tsimpri, Panagiota:

Untersuchung des Farbsehens mittels Pigmentfarbtafeln und Tablet-PC

2015, 75 Seiten, 39 Abbildungen, 14 Tabellen

\section{Kurzreferat:}

Im Rahmen einer prospektiven, randomisierten Studie wurde die Testung des Farbsehens bei angeborenen Farbsehstörungen bei Verwendung eines Buches (19 Pigmentfarbtafeln nach Velhagen/Broschman/Kuchenbecker) und eines Tablet-PCs (iPad2®) verglichen.

Die Untersuchung wurde unter standardisierten Untersuchungsbedingungen durchgeführt.

Es wurden in einem Zeitraum von Mai 2012 bis Dezember 2013 randomisiert 77 Probanden mit einem Durchschnittsalter von 42,81 $\pm 16,9$ und einem Durchschnittsvisus von 0,99 $\pm 0,1$ untersucht. 62 Probanden waren Farbnormale und 15 Probanden hatten eine angeborene Farbsehstörung. Die Übereinstimmung der Ergebnisse aller Probanden von Buch und Tablet-PC betrug 88.0\%. Bei den Probanden mit Farbsehstörungen bestand eine Übereinstimmung von $83,3 \%$, bei den Probanden ohne Farbsehstörungen bestand eine Übereinstimmung von 89,1\%. Zwischen Buch und $\mathrm{Pad1} \AA^{\circledR}$ bestand eine Übereinstimmung von $84.4 \%$ und zwischen iPad1 $\AA$ und $\mathrm{iPad} 2 \AA$ eine Übereinstimmung von $87,2 \%$.

Die vorliegenden Ergebnisse zeigen, dass bei der Untersuchung des Farbsehens in etwa vergleichbare Ergebnisse mittels Buch und Tablet-PC erzielt wurden, wobei die Trennschärfe des Buches besser war und die technische Limitationen des Tablet-PCs zu berücksichtigen sind.

\section{Schlüsselwörter:}

Farbsehen - Farbtafeln - Tablet-PC - iPad $2 \AA$ - angeborene Farbsehstörungen 
Meinen Eltern und meinem Mann in Dankbarkeit gewidmet. 


\section{Inhaltsverzeichnis}

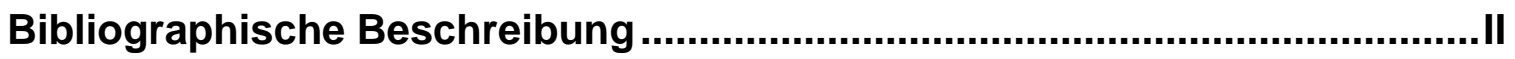

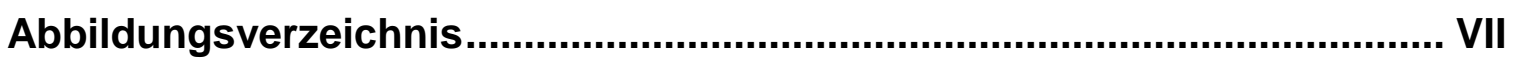

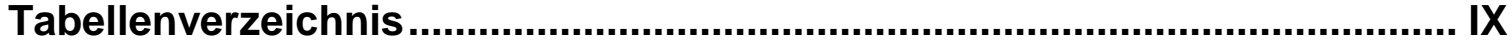

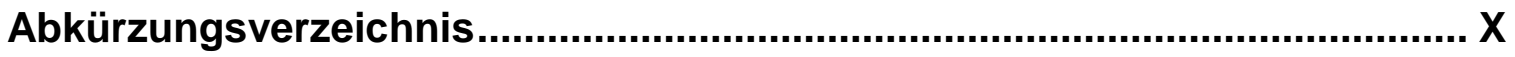

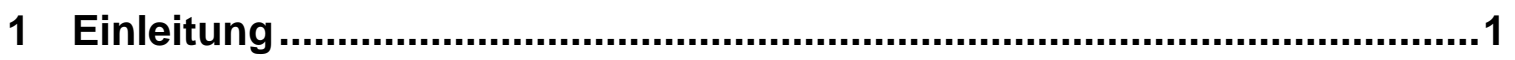

1.1 Nutzungsmöglichkeiten von Tablets-PC und Applikationen in der

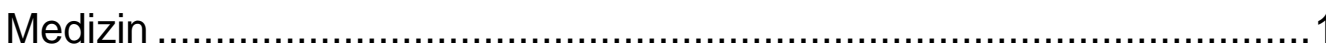

1.2 Der Einsatz von Smartphones und Tablet-PC in der

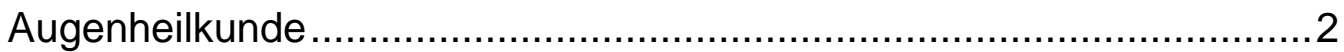

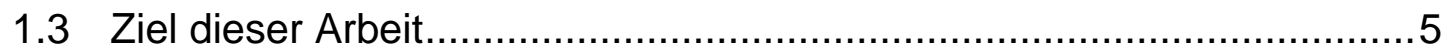

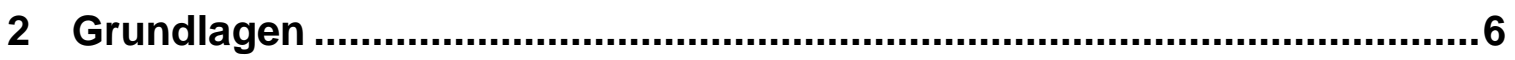

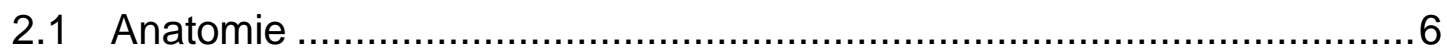

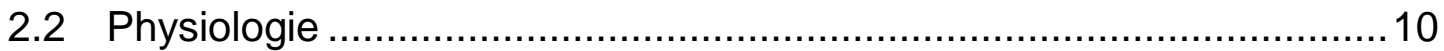

2.3 Angeborene und erworbene Farbsehstörungen ............................... 14

2.3.1 Angeborene Farbsehstörungen ......................................... 14

2.3.2 Erworbene Farbsehstörungen ............................................16

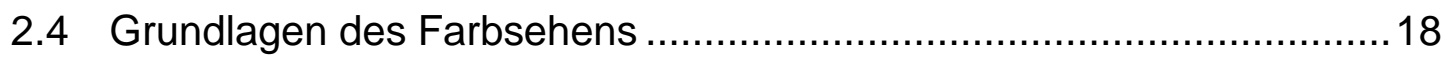

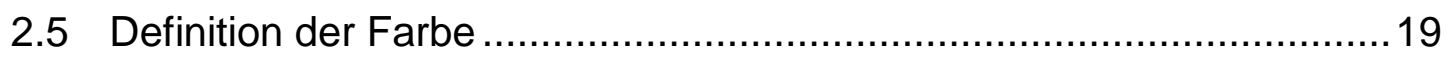

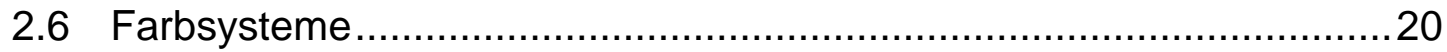

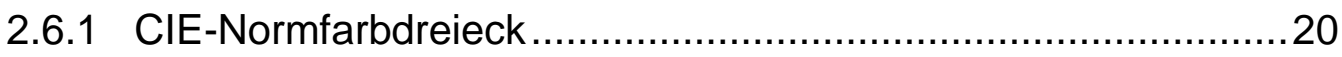

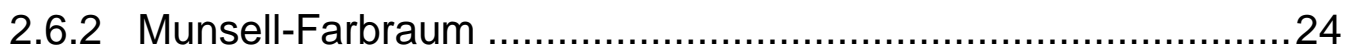

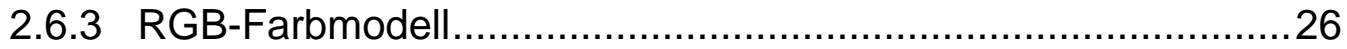

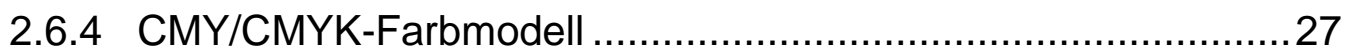

2.7 Untersuchungsmethoden bei Farbsehstörungen ...............................28

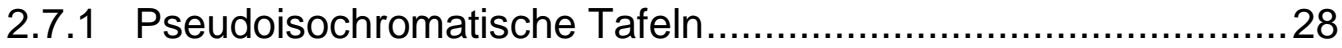

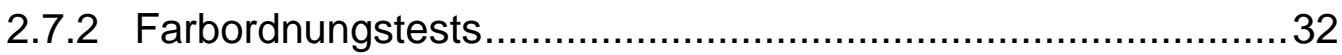

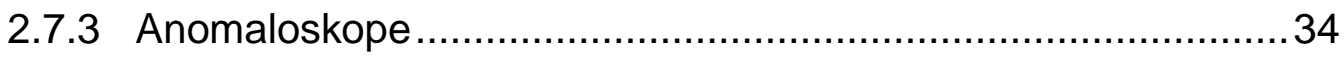

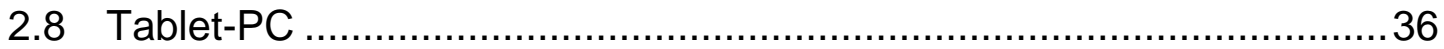




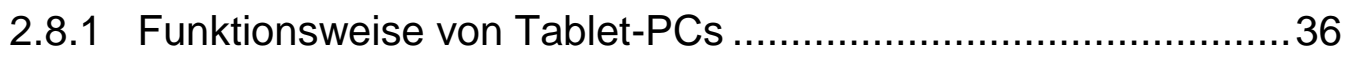

2.8.2 Farbdisplay von Tablet-PCs - Aufbau und Funktionsweise ......39

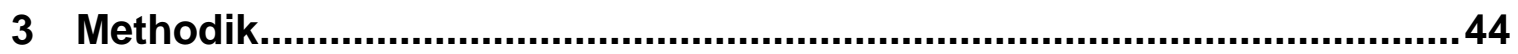

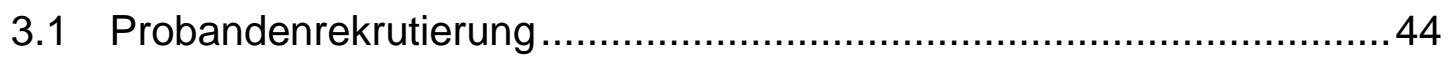

3.2 Verwendete Farbtafeln und verwendetes Anomaloskop .....................44

3.3 Durchführung der Tests ...............................................................46

3.4 Statistische Auswertung .......................................................... 48

4 Ergebnisse der statistischen Auswertung ….......................................50

4.1 Alters- und Geschlechtsverteilung ...............................................50

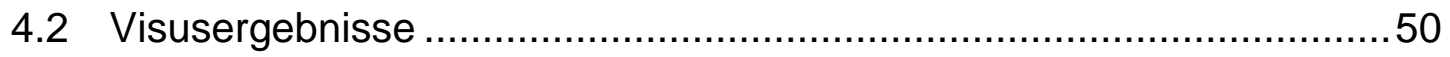

4.3 Verteilung der Farbsehstörungen .................................................

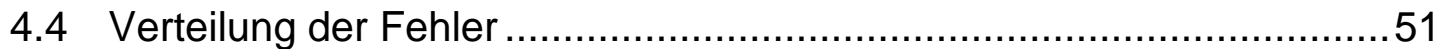

4.5 Vergleich des Farbsehens anhand verschiedener Medien ...................53

4.5.1 Vergleich des Farbsehens mittels Buch und iPad2® bei

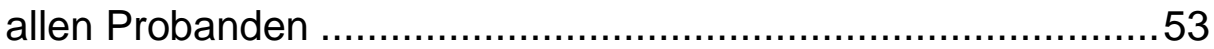

4.5.2 Vergleich des Farbsehens mittels Buch und iPad $2 \AA$ bei den

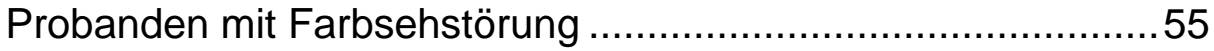

4.5.3 Vergleich des Farbsehens zwischen Buch und iPad2® bei den Probanden ohne Farbsehstörung ..................................57

4.5.4 Vergleich des Farbsehens mittels iPad1 $\AA$ und iPad2 $\AA$ bei allen Probanden

4.5.5 Vergleich bei der Untersuchung des Farbsehens zwischen Buch und iPad1® bei allen Probanden .......................................61

4.5.6 Vergleich bei der Untersuchung des Farbsehens zwischen den drei Gruppen (Buch vs. iPad1®, Buch vs. $\mathrm{iPad} 2 \AA$,

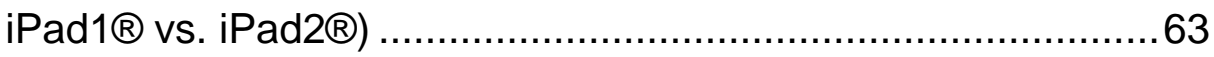

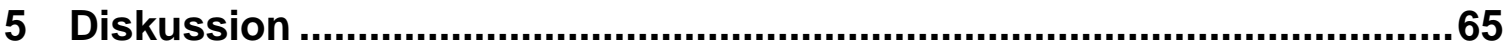

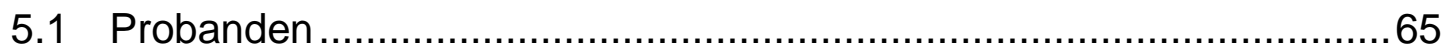

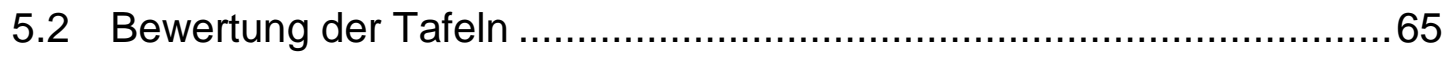

5.3 Technische Limitationen ...........................................................

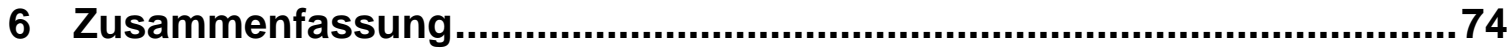


Literaturverzeichnis ................................................................................ XII

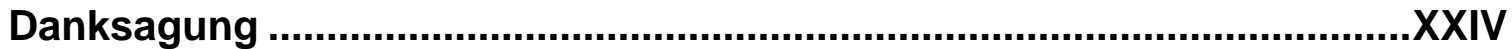

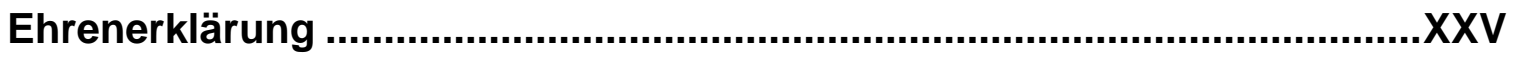

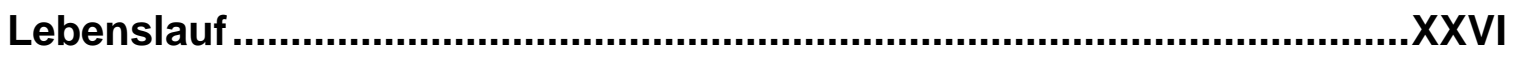

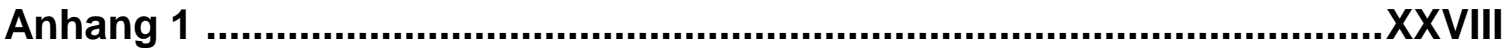

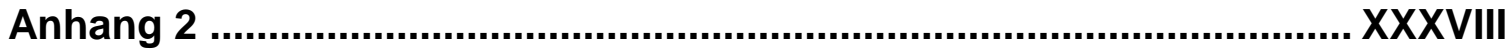

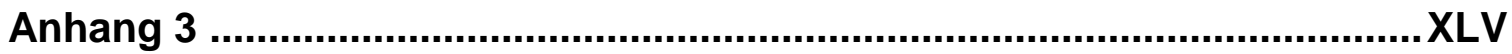




\section{Abbildungsverzeichnis}

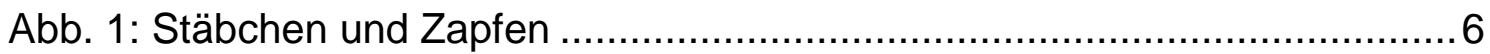

Abb. 2: Spektrale Absorptionskurven der menschlichen Rot-, Grün- und

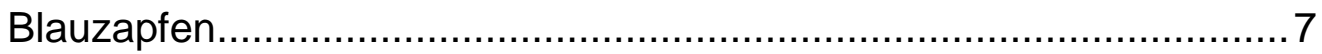

Abb. 3: Die Verteilung der Photorezeptoren in der Retina .............................. 8

Abb. 4: Simulation des Zapfenmosaiks der Fovea ......................................... 8

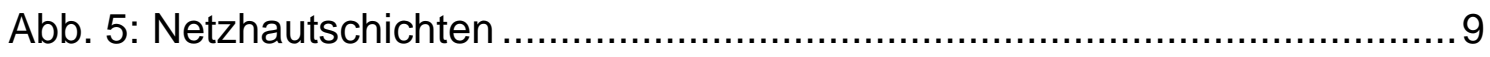

Abb. 6: Vergleich der Molekülstrukturen des Opsins ....................................10

Abb. 7: Stimulation der ON- und OFF-Kanäle ............................................11

Abb. 8: Synapsen zwischen Bipolar- und Ganglienzellen .............................. 12

Abb. 9: Schematische Darstellung der Projektion der Ganglienzellen am Corpus geniculatum im Colliculus superior .............................................. 13

Abb. 10: Unterschiedliche Farbtöne von Blau ............................................. 19

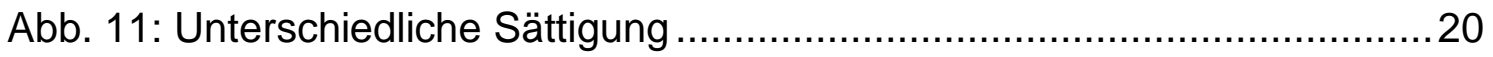

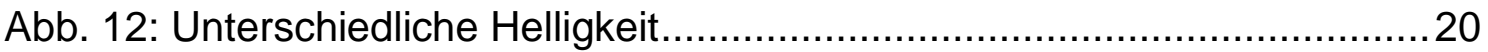

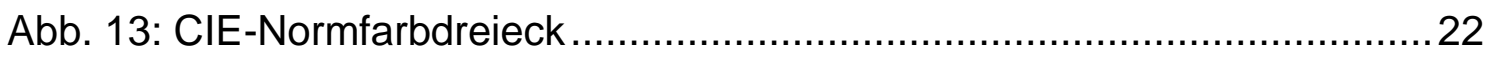

Abb. 14: Farbverwechselungsgeraden des Protanopen (a), Deuteranopen (b) und Tritanopen (c) .................................................................. 23

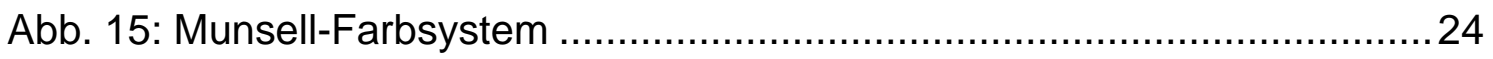

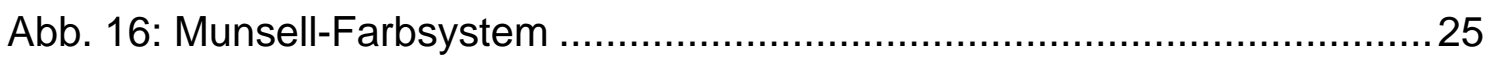

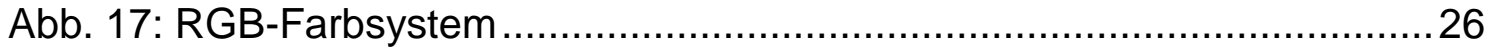

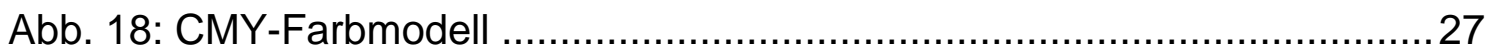

Abb. 19: Beispiel einer Transformationstafel .............................................29

Abb. 20: Beispiel einer Tafel mit verschwindenden Zeichen ...........................29

Abb. 21: Beispiel einer Tafel mit versteckten Zeichen ...................................30

Abb. 22: Beispiel einer diagnostischen Farbtafel ........................................ 30

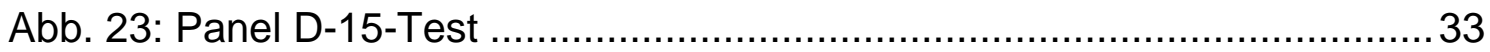

Abb. 24: Der Farnsworth-Munsell-100-Hue-Test ........................................... 33

Abb. 25: Kreisförmiges Prüffeld des HMC-Anomaloskops ................................. 34

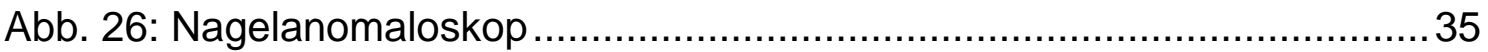




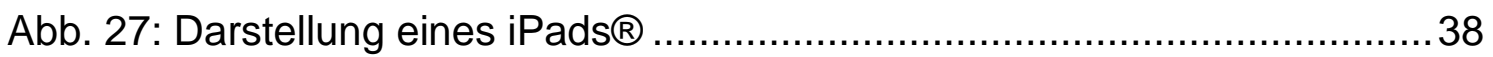

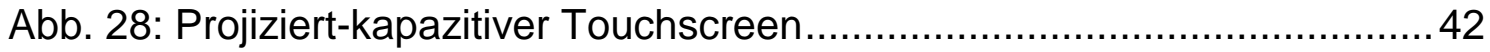

Abb. 29: iPad®-Farbraum vs. sRGB-Farbraum (Grid) .................................. 43

Abb. 30: Farbraumdarstellung von verschiedenen Tablet-PCs.......................43

Abb. 31: Tafeln zur Prüfung des Farbsehens ……....................................... 44

Abb. 32: Verwendetes HMC-Anomaloskop................................................. 46

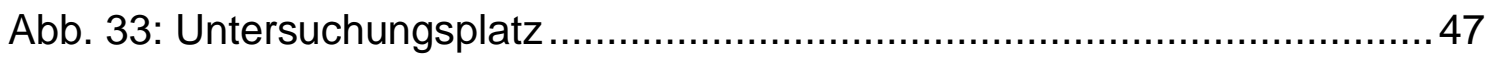

Abb. 34: Verwendetes iPad2® mit Darstellung einer Farbtafel ........................47

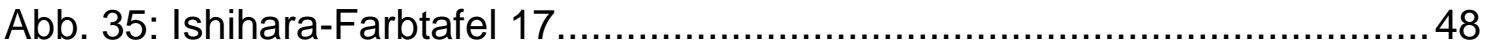

Abb. 36: Darstellung der Altersverteilung der Probanden mit und ohne

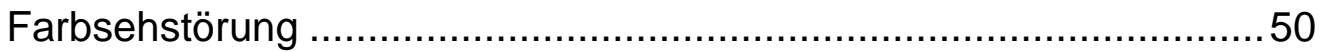

Abb. 37: Darstellung der Visusverteilung der Probanden mit und ohne

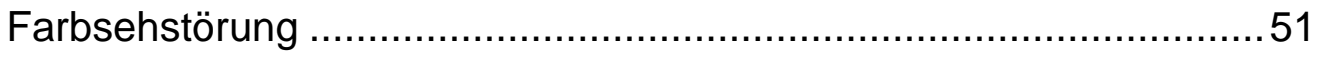

Abb. 38: Darstellung der Anzahl der Fehler pro Tafel bei der Untersuchung des Farbsehens mittels Buch bei den Probanden mit Farbsehstörung (rot) und ohne Farbsehstörung (blau) ..................................................... 52

Abb. 39: Darstellung der Anzahl der Fehler pro Tafel bei der Untersuchung des Farbsehens mittels iPad2® bei den Probanden mit Farbsehstörung (rot) und ohne Farbsehstörung (blau) .................................................. 52 


\section{Tabellenverzeichnis}

Tab. 1: Häufigkeit angeborener Farbsehstörungen..................................... 15

Tab. 2: Darstellung der Punktdichten (in Pixel pro Zoll) und Bildschirmauflösungen von verschiedenen iPad®-Generationen ........40

Tab. 3: Verwendete Farbtafeln. 45

Tab. 4: Vergleich des Farbsehens zwischen Buch und iPad2 $\AA$ bei allen

Probanden 53

Tab. 5: Auswertung der Ergebnisse pro Tafel zwischen Buch und iPad2® bei allen Nutzern

Tab. 6: Vergleich des Farbsehens mittels Buch und $\mathrm{iPad} 2 \AA$ bei den Probanden mit Farbsehstörung. 55

Tab. 7: Auswertung der Ergebnisse pro Tafel mittels Buch und iPad2® bei den Probanden mit Farbsehstörungen ...............................................56

Tab. 8: Vergleich der Untersuchung des Farbsehens zwischen Buch und iPad2® bei den Probanden ohne Farbsehstörungen

Tab. 9: Auswertung der Ergebnisse pro Tafel mittels Buch und iPad2® bei den Probanden ohne Farbsehstörungen 58

Tab. 10: Vergleich der Untersuchung des Farbsehens mittels iPad1® und iPad2® bei allen Probanden

Tab. 11: Auswertung der Ergebnisse pro Tafel mittels iPad1 1 und iPad2® bei allen Nutzern 60

Tab. 12: Vergleich des Farbsehens zwischen Buch und iPad1 $\AA$ bei allen 10 Probanden, die mittels iPad $1 \AA$ untersucht wurden

Tab. 13: Auswertung der Ergebnisse pro Tafel mittels Buch und iPad1® bei allen Nutzern, die mittels iPad1® untersucht wurden 62

Tab. 14: Übereinstimmung nach Landis und Koch zwischen den drei Gruppen (Buch vs. iPad2®, Buch vs. iPad1®, iPad1 $\AA$ vs. $\mathrm{iPad} 2 \AA$ ) 


\section{Abkürzungsverzeichnis}

\begin{tabular}{|c|c|}
\hline AAC & Advanced Audio Coding \\
\hline AAX & Avid Audio Extension \\
\hline AIFF & Audio Interchange File Format \\
\hline Abb. & Abbildung \\
\hline App & Applikation \\
\hline AV & audiovisuell \\
\hline bspw. & beispielsweise \\
\hline bzw. & beziehungsweise \\
\hline CD & Compact Disc \\
\hline CIE & Commission internationale de l'éclairage \\
\hline CMY/CMYK & Cyan Magenta Yellow/Cyan Magenta Yellow Key \\
\hline CMS & color management-system \\
\hline doc & document \\
\hline dt. & deutsch \\
\hline EDGE & Enhanced Data Rates for GSM Evolution \\
\hline EDR & Enhanced Data Rate \\
\hline etc. & et cetera \\
\hline $\mathrm{Fa}$ & Firma \\
\hline FDA & Food and Drug Administration \\
\hline GB & Gigabyte \\
\hline ggf. & gegebenenfalls \\
\hline $\mathrm{GHz}$ & Gigahertz \\
\hline gif & Graphics Interchange Format \\
\hline GSM & Global System for Mobile Communications \\
\hline $\mathrm{HD}$ & High Definition \\
\hline HDRI & High Dynamic Range Image \\
\hline HE-ACC & High-Efficiency Advanced Audio Coding \\
\hline
\end{tabular}




$\begin{array}{ll}\text { HSDPA } & \text { High-Speed Downlink Packet Access } \\ \text { HSUPA } & \text { High-Speed Uplink Packet Access } \\ \text { html } & \text { Hypertext Markup Language } \\ \text { Hz } & \text { Hertz } \\ \text { IPS } & \text { In-plane Switching } \\ \text { Jpg/JPEG } & \text { Joint Photographic Experts Group } \\ \text { LC } & \text { Low Complexity } \\ \text { LCD } & \text { Liquid-crystal Display } \\ \text { LED } & \text { Light-emitting Diode } \\ \text { MB } & \text { Megabyte } \\ \text { MHz } & \text { Megahertz } \\ \text { MPEG } & \text { Moving Picture Experts Group } \\ \text { nm } & \text { nanometer } \\ \text { Nr. } & \text { Nummer } \\ \text { OPN1LW } & \text { Opsin 1 Long Wave } \\ \text { OPN1MW } & \text { Opsin 1 Middle Wave } \\ \text { PC } & \text { Personal Computer } \\ \text { PCM } & \text { Puls-Code-Modulation } \\ \text { PCT/PCAP } & \text { Projected Capacitive Touch } \\ \text { PDA } & \text { Persönlicher digitaler Assistent } \\ \text { Pdf } & \text { Portable Document Format } \\ \text { ppi } & \text { Pixels per Inch } \\ \text { ppt } & \text { Power-Point-Presentation } \\ \text { PVC } & \text { Polyvinylchlorid } \\ \text { RAW } & \text { Raw image Format } \\ \text { RGB } & \text { Rot-Grün-Blau } \\ \text { RPE } & \text { retinales Pigmentepithel } \\ \text { SAW } & \text { Rich Text Format } \\ \text { Surface Acoustic Wave } \\ \text { OPA }\end{array}$


Sek. Sekunde

SIM Subscriber Identity Module

SOC System-on-a-chip

SPP Standard Pseudoisochromatic Plates

Tab. Tabelle

tiff Tagged Image File Format

TV Television

txt text

UMTS Universal Mobile Telecommunications System

USB Universal Serial Bus

usw. und so weiter

VBR Variable Bitrate

vcf Variant Call Format

VGA Video Graphics Array

vs. versus

WAN Wide Area Network

WLAN/Wi-fi Wireless Local Area Network

xls Excel File Format

z. A. zum Ausschluss

z. B. zum Beispiel 


\section{Einleitung}

\subsection{Nutzungsmöglichkeiten von Tablets-PC und Applikationen in der Medizin}

Mit der Entwicklung von Smartphones und Tablet-PC entstanden rasch auch viele medizinische Applikationen.

Es gibt heute kaum ein gesundheitliches oder medizinisches Problem, für das es keine Applikation gibt. In den letzten Jahren sind über 1500 Applikationen für den Gesundheitsbereich entwickelt worden. Die medizinischen Applikationen spielen derzeit in dem medizinischen Bereich nur eine untergeordnete Rolle, sie werden aber in den kommenden Jahren allmählich an Bedeutung zunehmen.

Es gibt bspw. die sogenannten „stand-alone-Apps“. Damit kann der Patient selbst seine Erkrankung dokumentieren und kontrollieren. Mit der Applikation „SkinTracer" kann der Patient z. B. verdächtige Hautläsionen dokumentieren und zur Begutachtung an Hautärzte senden.

Diabetiker können ein iPhone-Gadget benutzen, bei dem die Blutstreifen gelesen und dokumentiert werden können, wodurch die Dosierung der Insulinmenge justiert werden kann.

Zahlreiche Navigations-Apps sind von großem Nutzen im Alltag wie auch in Notfallsituationen. Sogar ein EKG kann heute mit dem Smartphone aufgenommen werden, das dann im Fall eines Herzinfarkts ein Signal absendet.

Die zunehmende Bedeutung der medizinischen Applikationen ist unbestritten. Bereits verfügbare bzw. modifizierte Applikationen werden vermutlich in der Zukunft als unabdingbare Hilfsmittel zur Unterstützung und Erleichterung im Gesundheitswesen beitragen (Stanzel et al., 2012). 


\subsection{Der Einsatz von Smartphones und Tablet-PC in der}

\section{Augenheilkunde}

Mit zunehmender Verbreitung von Smartphones und Tablet-PCs haben die Applikationen in der Augenheilkunde als Hilfsmittel zur Diagnose, Therapie und Bildung das Potenzial, eine Erleichterung in der klinischen Versorgung der Patienten, der Datenverarbeitung und der Kommunikation zu erzielen (Stanzel et al., 2012).

Die über 70 verfügbaren ophthalmologischen Applikationen können in verschiedene Kategorien unterteilt werden: klinische Untersuchungsfunktionen, Fotografie und Videoaufnahmen, Organisationsfunktionen, Erklärungs- und Aufklärungsfunktionen, medizinische Informationen.

Es gibt verschiedene ophthalmologische Applikationen für die Testung des Visus, des Astigmatismus, der Pupillengröße, zudem gibt es Amsler-Grid-Tests und ein Risikobewertungssystem für die Makuladegeneration (Chiu et al., 2014). Auch die Testung von anderen Sehfunktionen, wie z. B. die Farbsehtestung, ist mit Applikationen heute möglich. Zudem stehen auch Applikationen zur Farberkennung als Hilfsmittel bei Rot-Grün-Störung zur Verfügung. Weiterhin können Applikationen für die Berechnung des Glaukomrisikos, für das Glaukommonitoring und elektronische Patientenakten angewendet werden, zudem können Übersetzungs-Applikationen und Navigations-Applikationen für Sehbehinderte von Nutzen sein (Aslam et al., 2013; Lee et al., 2014; Ruamviboonsuk et al., 2012; Zhang et al., 2013). „The Eye Handbook“ ist z.B. eine sehr bekannte und weitverbreitete ophthalmologische Applikation mit vielen Nutzungsmöglichkeiten, von der Visusprüfung bis zur Kalkulation der IOL-Stärke (Lord et al., 2013). Eine Applikation zur Berechnung von Gesichtsfelddefekten erwies sich als präziser und schneller als die Interpretation durch oculoplastische Chirurgen. Die Beurteilung durch die Chirurgen war ungenau, sehr variabel und oft unterbewertet im Vergleich zu der Berechnung durch die Applikation. Der mittlere Prozentsatz der Fehler betrug 1,98\% bei der Applikation und 9,75\% bei den Chirurgen (R. N. Maamari et al., 2014). 
Die spektrometrische Funktion von Smartphones bzw. Tablet-Pcs kann preiswert für die Farbmetrik genutzt werden (Hong et al., 2014).

Es gibt auch verschiedene Methoden zur digitalen Fotodokumentation des anterioren und posterioren Abschnitts des Auges mittels Smartphone oder Tablet-PC. So gibt es die Möglichkeit der digitalen Fotodokumentation an der Spaltlampe mit einem modifizierten binokularen Strahlenteiler. Der erste Strahl ist mit einer digitalen Kamera und der zweite Strahl mit der Lichtquelle eines synchronisierten Blitzlichts verbunden. Dann gibt es die Möglichkeit der Fundusaufnahme mittels eines Monokulars am Mikroskop. Damit ist eine bis zu sechzigfache Vergrößerung möglich. Die dritte Variante ist ein USB-Mikroskop. Damit können Bilder mit hoher Auflösung aufgenommen und dann digital gespeichert werden. Eine ausgezeichnete Fotodokumentation ist für einen geübten Benutzer mit allen drei Methoden gut möglich. Die zunehmende Verbreitung von Smartphones und Tablet-PCs und die signifikante Verbesserung der digitalen Kameras machen ihren Einsatz in der Medizin ohne Zweifel sinnvoll (Tietjen et al., 2013). Mittels Smartphone und Tablet-PC können bspw. Vorderabschnitt- und Fundusfotos dokumentiert und weitergeleitet werden sowie eine Fluoreszenzangiographie durchgeführt werden. Außerdem kann ein Smartphone oder Tablet-PC als Datenbank genutzt werden (Barsam et al., 2010; Gurram, 2013; Haddock et al., 2013; Lamirel et al., 2012; Shanmugam et al., 2014; Suto, 2014a, 2014b). Eine Ophthalmoskopie mittels Smartphone zeigte ähnliche Ergebnisse mit der Spaltlampen-Biomikroskopie in Mydriasis bei der Untersuchung und Klassifikation der diabetischen Retinopathie. Die Tragbarkeit, die Erschwinglichkeit und die Netzwerkfähigkeit einer Ophthalmoskopie mittels eines Smartphone-Ophthalmoskops macht dies zu einer vielversprechenden Methode für Screening-Tests (Kumar et al., 2012; Russo et al., 2015). Außerdem können Fundusaufnahmen mittels einer smartphone-basierten retinalen Kamera bei der Diagnose, beim Monitoring und bei der Therapie von retinalen Erkrankungen bei Patienten mit eingeschränkter Mobilität durch die Möglichkeit der Fernauswertung (Telemedizin) sehr sinnvoll eingesetzt werden (R. N. Maamari et al., 2014). Smartphones wurden bereits zur Auswertung von 
Computertomographie-Bildern bei orbitaler Myocysticercose bei ambulanten Patienten verwendet (Meena et al., 2013).

Smartphones, Tablet-PCs und E-Books sind wichtige Hilfsmittel für Patienten mit reduzierter Sehschärfe. Patienten mit stark reduziertem Visus oder mit Erblindung können von Applikationen bzw. E-Books mit Spracheingabe- bzw. Sprachausgabe-Möglichkeiten profitieren. Objekte können aber auch mit der digitalen Kamera aufgenommen und mit der elektronischen Lupe vergrößert werden, was für Patienten mit relativ reduzierter Sehschärfe von großem Nutzen ist. Außerdem können mit dem Kamera-Lichtblitz Objekte besser beleuchtet werden (Crossland et al., 2014). Die Applikation „Display Reader“ zum Aufspüren von Haushalts- bzw. elektronischen Geräten für Patienten mit reduzierter Sehschärfe ist bereits in Entwicklung (Fusco et al., 2014). Auch ein spezielles sehkraft-basiertes computerassistiertes Smartphone-System zur Führung von Sehbehinderten an den Straßenkreuzungen ist bereits entwickelt worden. Das System ermöglicht die Selbstlokalisierung sowie die Einschätzung der relativen Lokalisation, bezogen auf die Fußgängerübergänge an den Straßenkreuzungen. Damit können sich Sehbehinderte auf den sicheren Fußgängerübergang ausrichten (Murali et al., 2013).

Auch gibt es kosteneffektive Applikationen, die mittels SMS die Patienten an Arzttermine rechtzeitig erinnern (Koshy et al., 2008).

Zusammenfassend werden Smartphones und Tablet-PCs zunehmend unverzichtbare Gegenstände im Alltag des Gesundheitswesens. Mit ophthalmologischen Applikationen können Smartphones auch zu anspruchsvollen Medizinprodukten werden. Smartphones können nützliche Instrumente für eine evidenzbasierte Medizin, für die Berufsausbildung, die klinische Mobilkommunikation, die Ausbildung und Aufklärung der Patienten, das Selbstmanagement von Erkrankungen, für das ferngesteuerte Monitoring von Patienten sein oder auch als administratives Mittel dienen (Chiu et al., 2014). Die innovative Rolle der medizinischen Applikationen für die Forschung, Ausbildung und Datenübertragung zeigt, dass Smartphones und Tablet-PCs in der Zukunft 
eine große Rolle in der Augenheilkunde und der Medizin allgemein spielen können (Chhablani et al., 2012; Zvornicanin et al., 2014).

Die Nutzung von Smartphones und Tablet-PCs als medizinische Produkte erfolgt derzeit aber nicht standardisiert, und die Verwendung im klinischen Alltag sollte vorsichtig überlegt werden (Zvornicanin et al., 2014). In einer Studie zu 182 ophthalmologischen Applikationen waren nur 37\% professionell konzipiert worden. Davon waren jeweils nur 52\% der Applikationen von Ophthalmologen und $44 \%$ von Optikern entworfen worden. 31\% der untersuchten Applikationen waren nicht von Ophthalmologen und $21 \%$ überhaupt nicht von Experten erstellt worden (Cheng et al., 2014).

Für die Farbsehtestung sind bereits zahlreiche Computerprogramme und webbasierte Anwendungen entwickelt worden (Arden et al., 1988; Awad et al., 2007; Cavanagh et al., 1986; Edsel et al., 1994; Heard et al., 1987; Hoffmann et al., 1998; Ing et al., 1994; Kuchenbecker et al., 2007; Mollon et al., 1989; Reffin et al., 1991; Röhl et al., 2007). Auch finden sich in einigen Applikationen für Smartphones bzw. Tablet-PCs zur Farbsehtestung Farbtafeln, wie z. B. „The Eye Handbook" oder "Eye2Phone“, die jedoch bisher nicht in Studien auf ihre Tauglichkeit untersucht worden sind.

\subsection{Ziel dieser Arbeit}

Es gibt derzeit noch keine Applikationen bzw. E-Books für die Testung des Farbsehens, die durch Studien evaluiert sind. Ziel unserer Studie ist der Vergleich der Untersuchungsergebnisse zwischen Tablet-PC und Buch (34. Auflage der Broschmann/Velhagen/Kuchenbecker-Farbtafeln), um eine Aussage treffen zu können, inwieweit sich ein Tablet-PC (iPad®) für eine Prüfung bzw. das Screening des Farbsehens eignet. 


\section{Grundlagen}

\subsection{Anatomie}

Im menschlichen Auge existieren zwei Grundtypen von Photorezeptoren: die Stäbchen und die Zapfen (Abb. 1).

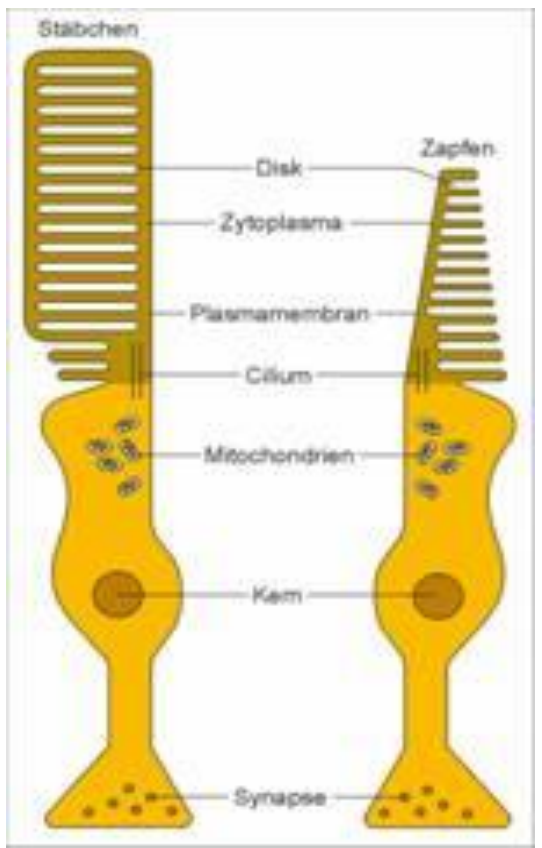

Abb. 1: Stäbchen und Zapfen (Frings et al., 1998)

Die 110-125 Millionen Stäbchen enthalten das Photopigment Rhodopsin und sind für das blaue-grüne Licht empfindlich. Die Stäbchen ermöglichen das Dämmerungs- (mesopisches Sehen) und das Nachtsehen (skoptopisches Sehen). Die 6-7 Millionen Zapfen enthalten verschiedene Typen des Farbpigments Opsin. Sie sind für das Tagessehen (photopisches Sehen) sowie für das Auflösungsvermögen und das Farbsehen zuständig. Abhängig von der Struktur des Opsin-Moleküls kann man drei Zapfentypen unterscheiden: Blau-, Grün- und Rotzapfen. Die Blauzapfen enthalten ein kurzwelliges Farbpigment (Cyanlab), welches den spektralen Bereich mit dem Empfindlichkeitsmaxima 415-425 nm erfasst, die Grünzapfen sind mittelwellig (Chlorolab) und erfassen den spektralen Bereich mit dem Empfindlichkeitsmaxima 520-540 nm, und die Rotzapfen (Erythrolab) sind langwellig und erfassen den spektralen Bereich mit 
dem Empfindlichkeitsmaximum 550-570 nm (Boynton, 1988; Conway, 2009; De Valois et al., 1966; Gegenfurtner et al., 2003; Jacobs, 1976; Lanthony, 1987; Lennie et al., 1988; Michael, 1973; Nathans, 1989, 1999; Nathans et al., 1989; Nathans, Merbs, et al., 1992; Nathans, Piantanida, et al., 1986; Ripps et al., 1969; Swanson et al., 2003; Walraven, 1972) (Abb. 2) .

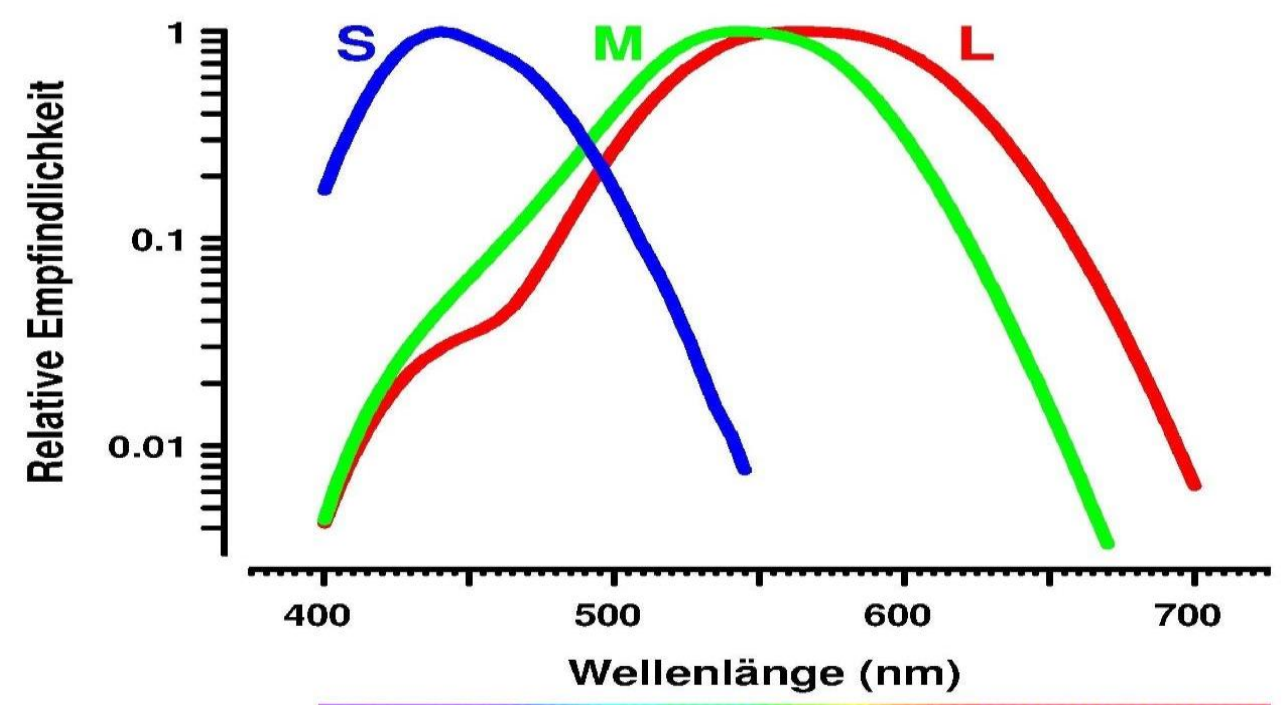

Abb. 2: Spektrale Absorptionskurven der menschlichen Rot-, Grün- und Blauzapfen (Gegenfurtner et al., 2003)

Die Zapfen sind auf der Netzhaut zu einem unregelmäßigen Mosaik angeordnet. Die Zapfen haben die höchste Dichte im Zentrum der Fovea centralis, und zur Peripherie hin nimmt die Dichte rasch ab. Bei einer Exzentrizität von $6^{\circ}$ steigt die Anzahl der Blauzapfen um bis zu 250\% an, während der Anteil der Rot- und Grünzapfen um 30\% fällt (Marré et al., 1986). Blauzapfen gibt es nur in der peripheren Retina, aber auch dort treten sie mit einer geringeren Dichte auf. Die höchste Dichte der Stäbchen findet sich in ca. $20^{\circ}$ Abstand von der Fovea. Grundsätzlich ist die Stäbchendichte in der Peripherie viel höher. Am blinden Fleck (ca. $15^{\circ}$ ) gibt es keine Rezeptoren (Abb. 3). 


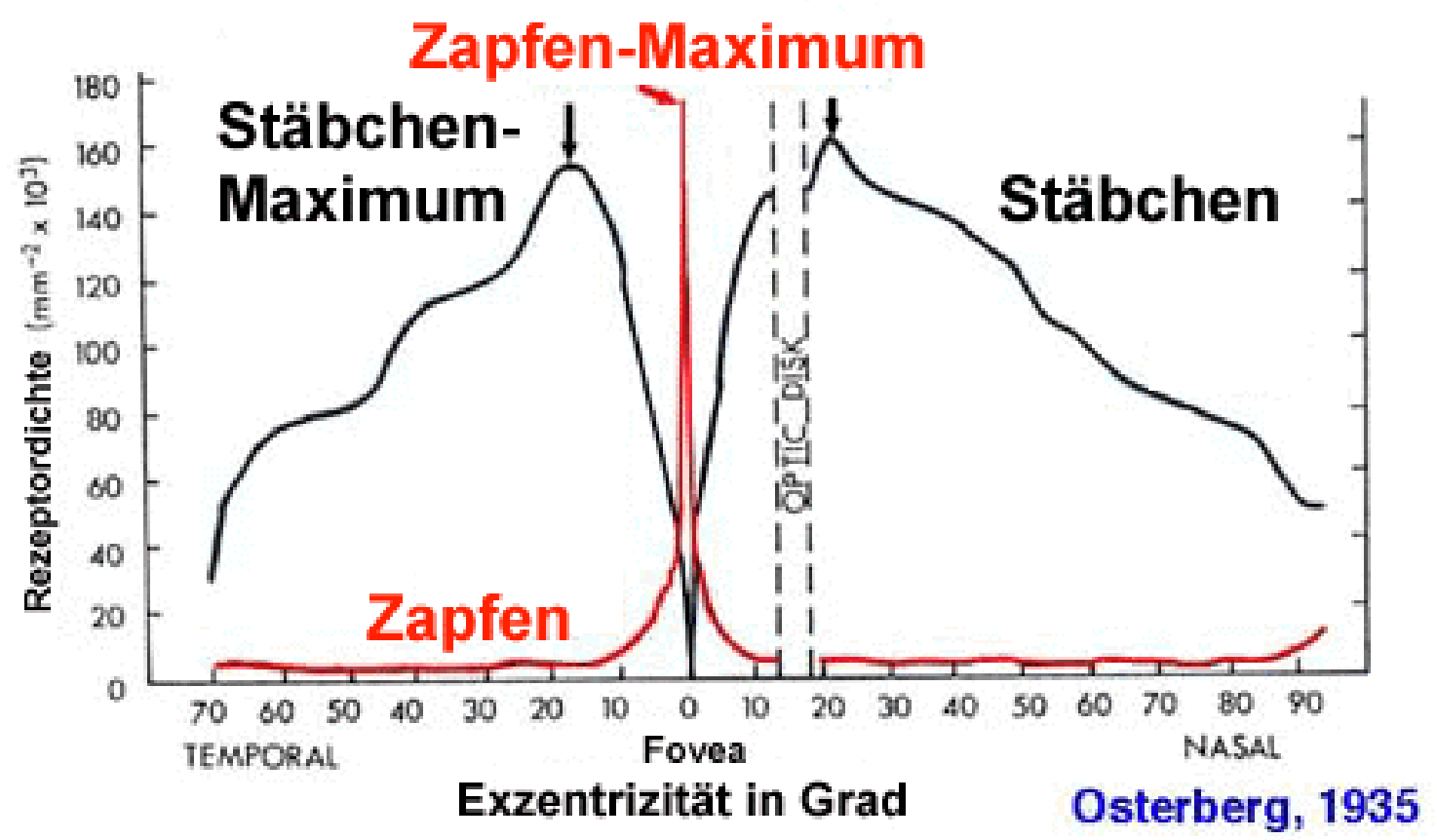

Abb. 3: Die Verteilung der Photorezeptoren in der Retina (Kolb, 1995e; Osterberg, 1935)

Die Rot-zu-Grün-Zapfen-Ratio kann variieren (Carroll et al., 2002; Yamaguchi et al., 1997). Die Rotzapfen kommen in der Regel häufiger vor. Es gibt etwa elf langwellig empfindliche Zapfen, neun mittelwellig und nur einen kurzwellig empfindlichen Zapfen (Dartnall et al., 1983). Das Photorezeptormosaik der menschlichen Netzhaut wurde 1999 direkt abgebildet (Roorda et al., 1999) (Abb. 4).

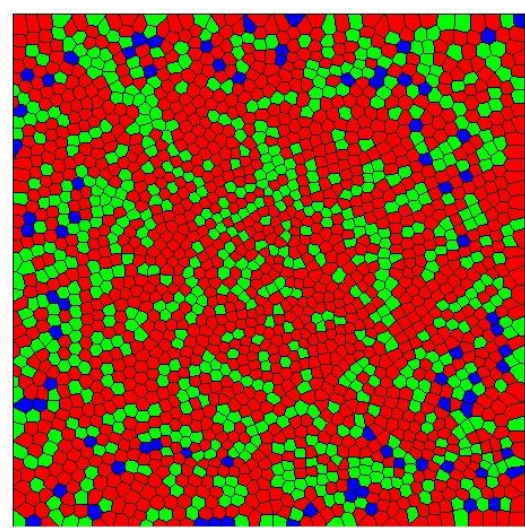

Abb. 4: Simulation des Zapfenmosaiks der Fovea. Die Positionen der Zapfen entstammen anatomischen Messungen. Die Einfärbung wurde nach einem Zufallsschema durchgeführt unter der Annahme, dass Rotzapfen ca. doppelt so häufig sind wie Grünzapfen (Gegenfurtner et al., 2010). 
Die Photorezeptorenaußensegmente liegen am retinalen Pigmentepithel und sind mit einer kleinen Übergangszone mit dem Photorezeptorinnensegment verbunden. Die Innensegmente sind mit den Müller-Zellen verbunden (Abb. 5).

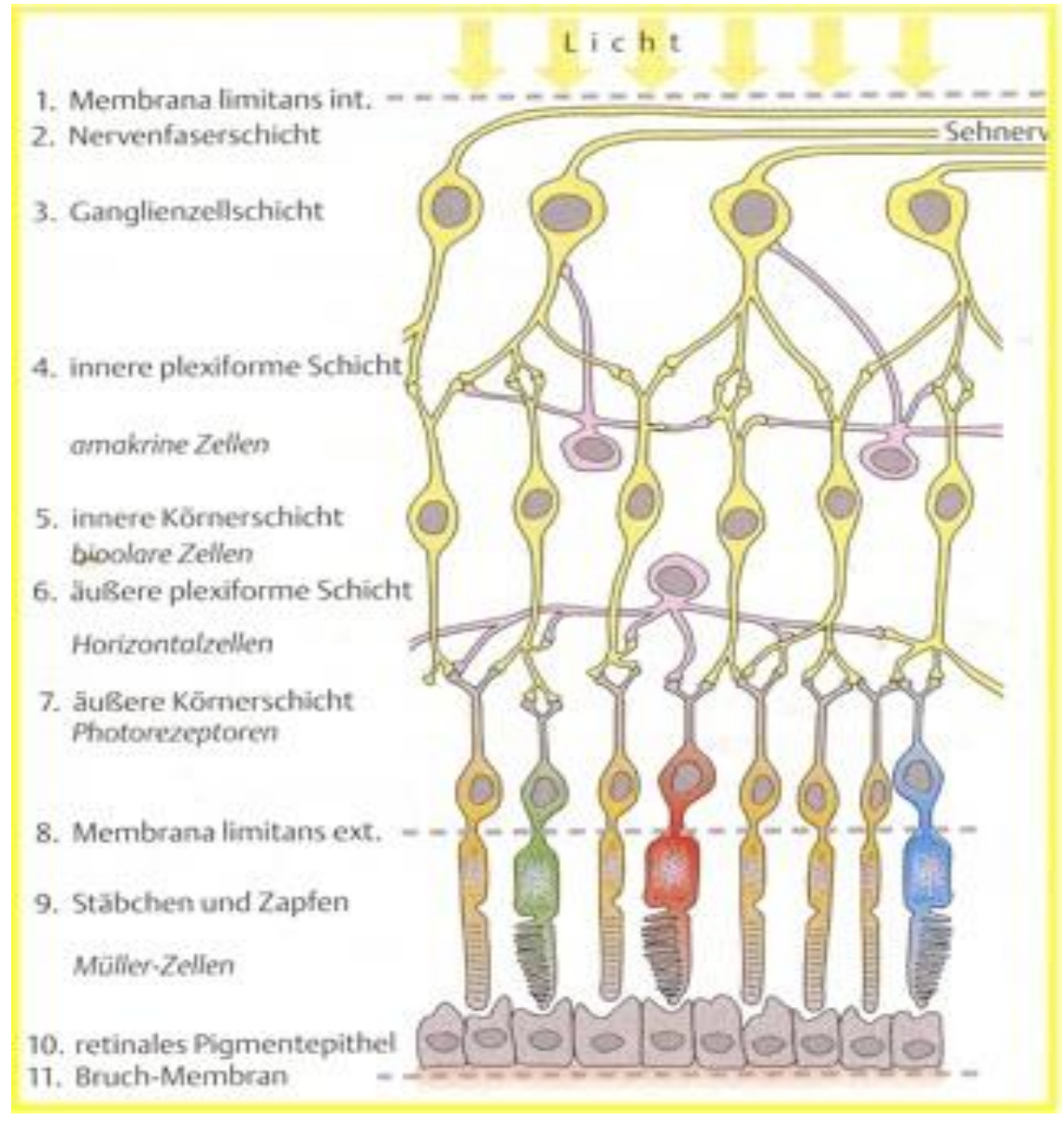

Abb. 5: Netzhautschichten (Lang, 2000)

Der Interphotorezeptormatrix füllt den Interzellulärraum zwischen den Photorezeptorenaußensegmenten und gewährleistet teilweise die Adhäsion zwischen Netzhaut und RPE. In den Mikrovilli der RPE-Zellen findet die Phagozytose der äußeren Anteile der Außensegmente statt. In den Innensegmenten befinden sich der Zellkern und die Dendriten, welche mit den Bipolar- und den Horizontalzellen Synapsen bilden. Die Außensegmente werden ständig regeneriert (Kolb, 1995e, 1995h). 


\subsection{Physiologie}

\section{$\underline{\text { Intraretinale Signalverarbeitung }}$}

Wenn ein Farbreiz die Netzhaut trifft, wird dieser zunächst von den Photorezeptoren absorbiert. Die Opsine (Rhodopsin, Blau-, Grün- und RotOpsin) der Photorezeptoren sind in die Membranscheiben der Außensegmente eingebunden. Die Aminosäuresequenz jedes Opsin-Moleküls ist für dessen spezifische spektrale Absorptionseigenschaften verantwortlich (Nathans, 1999; Nathans, Piantanida, et al., 1986; Nathans, Thomas, et al., 1986) (Abb. 6).
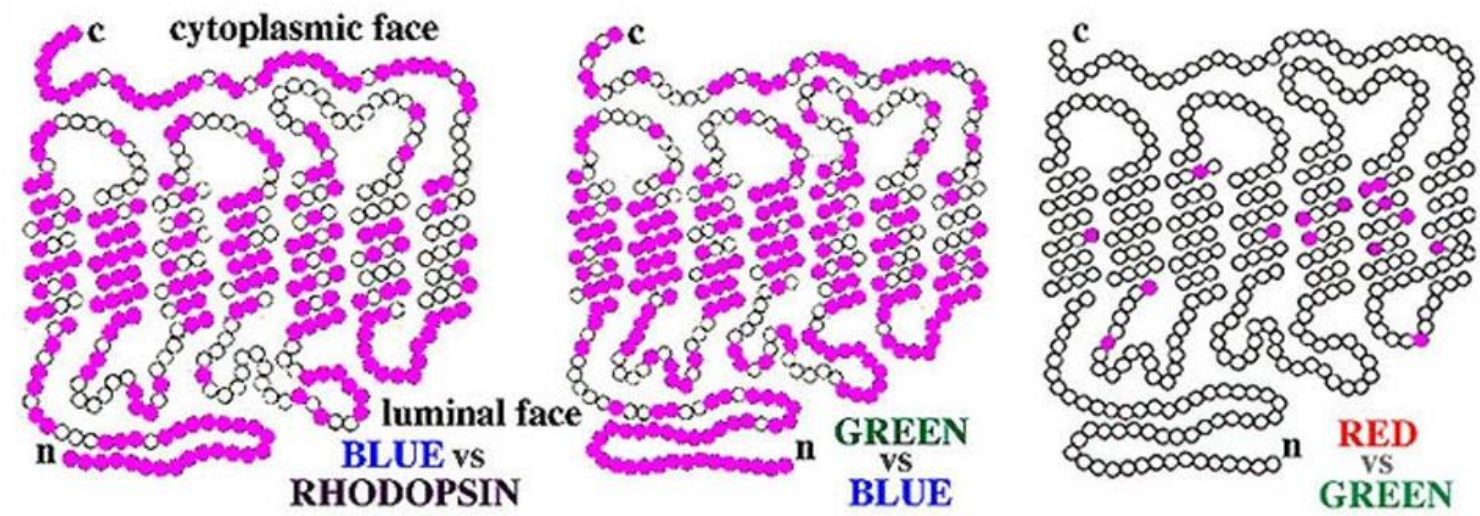

Abb. 6: Vergleich der Molekülstrukturen des Opsins. Links: Blau-Opsin vs. Rhodopsin, in der Mitte: Blau- vs. Grün-Opsin, rechts: Grün- vs. Rot-Opsin (farbige Punkte markieren die unterschiedlichen Aminosäuren, weiße Punkte die identischen Aminosäuren) (Nathans, Thomas, et al., 1986)

Jedes Opsin ist mit einem Vitamin-A-Derivat (11-cis-Retinal) gekoppelt. Nach Resorption eines Photons wandelt sich das 11-cis-Retinal in ein all-trans-Retinal um, somit wird das Opsin aktiviert und löst die Transduktionskaskade aus (Kolb, 1995e, 1995g). Nach Lichtstimulus werden die Außensegmente der Photorezeptoren hyperpolarisiert. Das Signal setzt sich über die Zellmembran zu den Innensegmenten fort. Die Photorezeptoren sind mit Bipolarzellen und Horizontalzellen verbunden. Die Aktivität der benachbarten Photorezeptoren wird durch die Signale der Horizontalzellen moduliert. Verschiedene Verbindungen zwischen den Photorezeptoren führen zu einem direkten Informationsaustausch 
zwischen den umgebenden Stäbchen und Zapfen. Wenn ein Zapfen polarisiert wird (Hyperpolarisation bzw. Depolarisation), dann werden die Bipolarzellen stimuliert, welche entweder mit OFF-Center-Ganglienzellen oder mit ON-CenterGanglienzellen verbunden sind. Das Licht führt zur Hyperpolarisation eines Zapfens sowie der ON-Bipolarzellen. Im Dunkeln kommt es zu einer Depolarisation eines Zapfens sowie der OFF-Bipolarzellen. Beim Übergang von Hell zu Dunkel werden die ON-Bipolarzellen gehemmt und die OFF-Bipolarzellen gleichzeitig aktiviert. Dieses ist möglich, weil ON- und OFF-Bipolarzellen verschiedene Rezeptoren, aber den gleichen Transmittor haben (Abb.7).

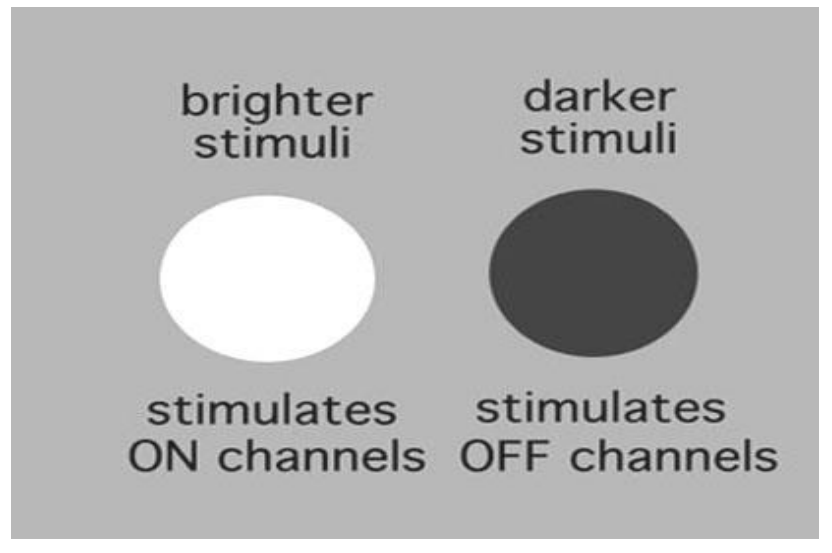

Abb. 7: Stimulation der ON- und OFF-Kanäle (Kolb, 1995b)

Es kommt dann zu einer spatialen und spektralen Interaktion von unterschiedlichen Interneuronen: den Bipolarzellen, den Amakrinzellen und den Horizontalzellen.

Die drei verschiedenen Horizontalzellen spielen nur eine Inhibitionsrolle. Ihre Hyperpolarisation bewirkt eine laterale Hemmung der kontaktierten Photorezeptoren und führt somit zu einer Signalverstärkung eines einzelnen Photorezeptors.

Die elf verschiedenen Typen von Bipolarzellen leiten das Signal aus den aktivierten Photozeptoren und Horizontalzellen in verschiedene ON- und OFFKanäle. Für die Stäbchen gibt es spezifische Stäbchen-ON-Bipolarzellen. Für die Rot- und Grünzapfen gibt es die depolarisierten Zapfen-ON-Bipolarzellen und die hyperpolarisierten Zapfen-OFF-Bipolarzellen. Im Zentrum besteht eine Eins-zu- 
Eins-Verbindung zwischen Photorezeptoren und Bipolarzellen, wobei zur Peripherie hin mehrere Zapfen mit weniger Bipolarzellen Synapsen bilden. Für die Blauzapfen gibt es spezifische Blauzapfen-ON-Bipolarzellen. Die Existenz von OFF-Bipolarzellen für Blauzapfen ist umstritten (Kolb, 1995a, 1995b, 1995c, 1995d; Kolb et al., 1996).

Es sind 30 Typen von Amakrinzellen beschrieben. Die Hauptfunktion ist die intraretinale Informationsverarbeitung durch Synapsen mit Bipolar- und Ganglienzellen. Des Weiteren tragen die Amakrinzellen zur Modifizierung von Farb-, Kontrast-, Helligkeits- und Bewegungsinformation bei (Kolb, 1995f).

\section{Postrezeptorale Signalverarbeitung}

Man kann achtzehn verschiedene Typen von Ganglienzellen unterscheiden. Die Ganglienzellen kontaktieren ON- und OFF-Bipolarzellen (Abb. 8).

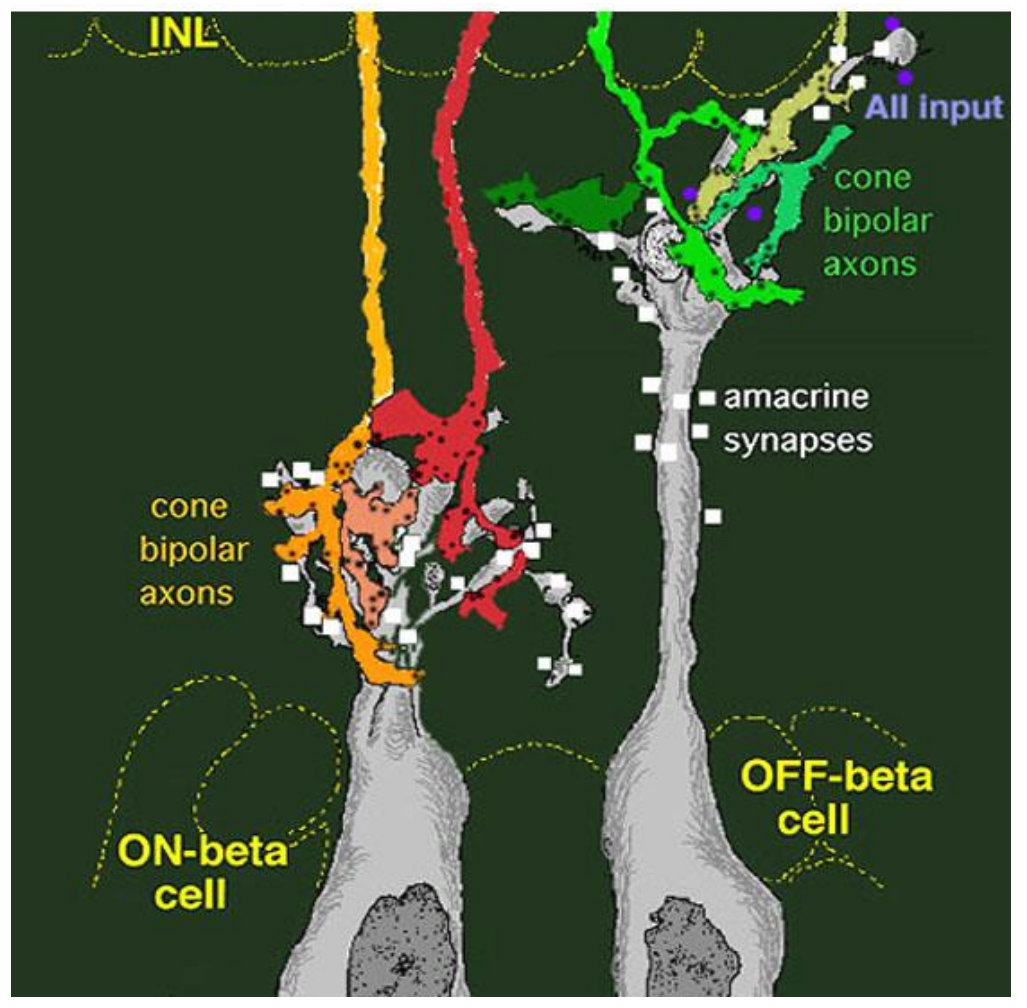

Abb. 8: Synapsen zwischen Bipolar- und Ganglienzellen (Kolb, 1995b)

Daher gibt es entsprechend ON- und OFF-Ganglienzellen, welche weiterhin die Information über den Sehnerv und das Chiasma opticum zum Corpus 
geniculatum vermitteln. Die ON-Ganglienzellen leiten die Information „Licht heller" und die OFF-Ganglienzellen die Information "Licht dunkler" weiter. Dazu kann man zwei weitere spezifische Ganglienzellentypen unterscheiden: die tonischen oder parvozellulären und die phasischen oder magnozellulären Ganglienzellen. Die parvozellulären Ganglienzellen sind mehr farbantagonistisch und zeigen eine niedrige Nervenleitungsgeschwindigkeit, eine hohe räumliche und eine niedrige zeitliche Auflösung. Die magnozellulären Ganglienzellen sind weniger farbantagonistisch, zeigen eine höhere Nervenleitgeschwindigkeit sowie eine niedrige räumliche und eine hohe zeitliche Auflösung (Gouras, 1968, 1969).

Das Corpus geniculatum enthält sechs Schichten (4 parvozelluläre und 2 magnozelluläre), je drei für jedes Auge. Die P-Zelltypen übermitteln sowohl Farbinformation als auch Helligkeitsinformation. Die M-Zelltypen übermitteln ausschließlich Helligkeitsinformation (De Valois et al., 1967). Das Signal wird im Verlauf vom Corpus geniculatum weiter über die Sehstrahlung zur Area striata an den primären visuellen Cortex geleitet. Das phasische System projiziert zusätzlich zum Colliculus superior und in andere visuelle Kerne (Erb et al., 2006) (Abb. 9).

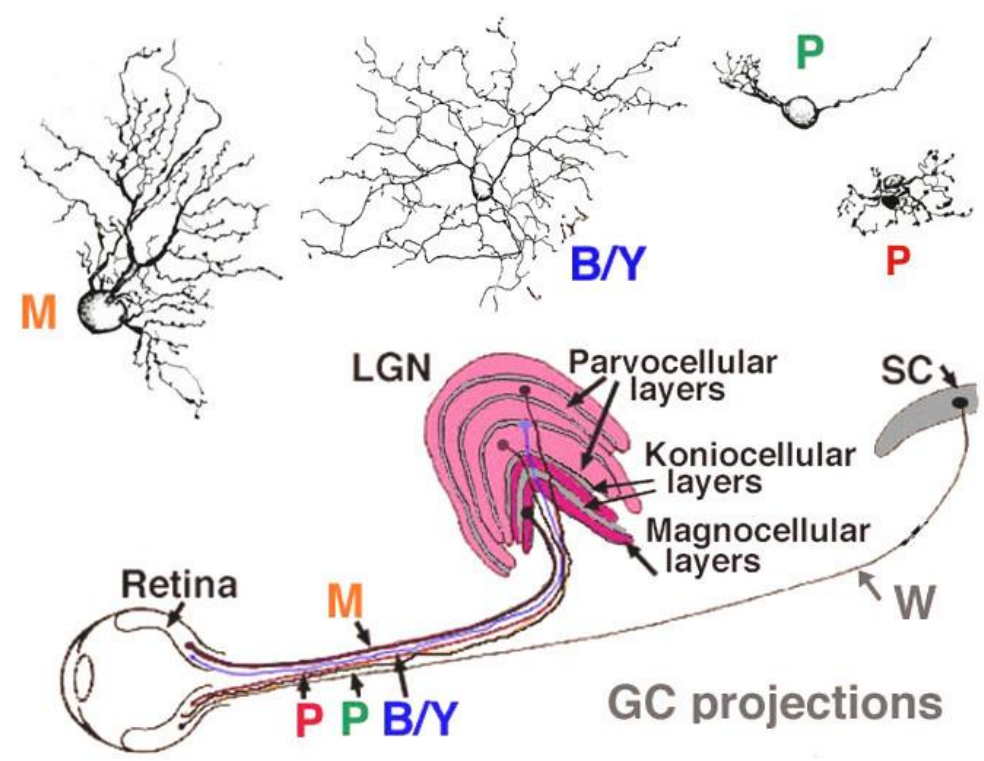

Abb. 9: Schematische Darstellung der Projektion der Ganglienzellen am Corpus geniculatum im Colliculus superior (Kolb, 1995c) 


\subsection{Angeborene und erworbene Farbsehstörungen}

Man unterscheidet die kongenitalen Farbsehstörungen, welche genetisch bedingt sind, von solchen, die durch Schädigung der brechenden Medien, der Netzhaut oder des Sehnervs entstehen und zu den erworbenen Farbsehstörungen gehören.

\subsubsection{Angeborene Farbsehstörungen}

Die angeborenen Farbsehstörungen kann man in drei Kategorien einteilen:

a. Monochromasie (Achromatopsie congenita)

Bei den Monochromaten fehlen alle Zapfenpigmente. Dabei wird von den Betroffenen nur Schwarz und Weiß wahrgenommen, es werden keine bunten Farben erkannt.

b. Dichromasie

Dabei werden zwei Farbengruppen sowie Schwarz und Weiß mit Graustufen erkannt. Die Betroffenen kann man weiterhin in drei Gruppen unterteilen:

- Protanopen sind diejenigen, bei denen das langwellige Zapfenpigment fehlt und die die rote Farbe nicht wahrnehmen (Rotblindheit).

- Deuteranopen sind diejenigen, bei denen das mittelwellige Zapfenpigment fehlt und die die grüne Farbe nicht wahrnehmen (Grünblindheit).

- Tritanopen sind diejenigen, bei denen das kurzwellige Zapfenpigment fehlt und die die blaue Farbe nicht wahrnehmen (Blaublindheit).

c. anomale Trichromasie

Hierbei werden viele Farben aus allen Bereichen wahrgenommen, aber eine der drei Komponenten ist unterwertig. Es gibt drei Gruppen der anomalen Trichromasie:

- Protanomalie („Rotschwäche“)

- Deuteranomalie („Grünschwäche“) 
- Tritanomalie („Blauschwäche“)

In der Praxis können die Dichromaten und die von Geburt an Trichromaten in drei Gruppen zusammengefasst werden:

- $\quad$ Protanope und Protanomale $=$ Protane

- Deuteranope und Deuteranomale = Deuterane

- Tritanope und Tritanomale = Tritane (Deeb et al., 1993; Motulsky, 1988; Nathans, Sung, et al., 1992; Neitz et al., 2000; Sharpe et al., 1998)

\section{Epidemiologie und Vererbung}

In Westeuropa sind 8\% der Männer und nur 0,4-0,5\% der Frauen von einer angeborenen, x-chromosomal rezessiven Rot-Grün-Farbfehlsichtigkeit betroffen. Allerdings sind $15 \%$ der Frauen heterozygote Überträgerinnen. Von den Betroffenen sind in der männlichen Bevölkerung ca. 5\% Deuteranomale und je ca. 1\% Protanomale, Protanope und Deuteranope. Bei den Schwarzafrikanern sind ca. 3\% der Männer und bei den Chinesen und den Japanern ca. 5\% von einer Farbfehlsichtigkeit betroffen (Birch, 2012; Deeb et al., 1993; Krastel, 1995).

Die angeborenen Tritanen sind mit einer Häufigkeit im Bereich von 1:10000 bis 1:60000 sehr selten (Kalmus, 1955b). Es handelt sich um eine Mutation des Gens auf Chromosom 7, welches das Blauzapfenfotopigment (Cyanlab) dekodiert. Die Mutation wird autosomal dominant vererbt (Weitz, Miyake, et al., 1992; Weitz, Went, et al., 1992) (Tab. 1).

Tab. 1: Häufigkeit angeborener Farbsehstörungen (in Anlehnung an (Cole, 1972; Pease, 1998; Wright, 1952))

\begin{tabular}{|l|l|l|}
\hline Art der Farbsehstörung & Männer & Frauen \\
\hline Insgesamt & $\sim 8 \%$ & $\sim 0,5 \%$ \\
\hline Anomale Trichromasie & & \\
\hline Protanomalie & $1 \%$ & $0,01 \%$ \\
\hline Deuteranomalie & $5 \%$ & $0,4 \%$ \\
\hline Tritanomalie & $0,001 \%$ & $0,001 \%$ \\
\hline Dichromasie & & \\
\hline Protanopie & $1 \%$ & $0,01 \%$ \\
\hline Deuteranopie & $1,5 \%$ & $0,01 \%$ \\
\hline
\end{tabular}




\begin{tabular}{|l|l|l|}
\hline Tritanopie & $0,008 \%$ & $0,008 \%$ \\
\hline Monochromasie & & \\
\hline Stäbchenmonochromasie & selten & selten \\
\hline Zapfenmonochromasie & selten & selten \\
\hline $\begin{array}{l}\text { Atypische } \\
\text { Monochromasie }\end{array}$ & sehr selten & sehr selten \\
\hline
\end{tabular}

Die beiden Gene, mit denen die Rot-Grün-Farbsehstörungen zusammenhängen, sind das OPN1LW („Opsin 1 Long Wave“), welches das Farbphotopigment Erythrolab kodiert, und OPN1MW („Opsin 1 Middle Wave“), welches das Farbphotopigment Chlorolab kodiert. Etwa 75\% der Rot-Grün-Farbsehstörungen (100\% aller Protanfarbfehlsichtigen und etwa 65\% aller Deutanfarbfehlsichtigen) können mittels molekulargenetischer Testung dieser Gene ermittelt werden. Die Stäbchenmonochromasie wird autosomal rezessiv vererbt. Dafür sind Mutationen in CNGA3 in Chromosom 2, in CNGB3 in Chromosom 8 und in GNAT2 in Chromosom 1 verantwortlich (Deeb et al., 1993, 1996).

\subsubsection{Erworbene Farbsehstörungen}

Farbsehstörungen sind ein häufiges Begleitsymptom bei Erkrankungen der Netzhaut oder der aufsteigenden Sehbahn. Nach der Köllnerschen Regel führen (Köllner, 1912) Defekte der distalen Retina meistens zu Blau-GelbFarbsehstörungen, wobei Defekte in der aufgestiegenen Sehbahn hauptsächlich Rot-Grün-Farbsehstörungen zur Folge haben. Die erworbenen Farbsehstörungen können nach Verriest (Verriest, 1963) in drei Typen unterteilt werden. Der Typ I ist eine erworbene Rot-Grün-Störung. Der Typ II ist eine erworbene Rot-Grün-Störung, die jedoch von einer Blau-Gelb-Störung begleitet wird. Der Typ III ist eine erworbene Blau-Gelb-Farbsehstörung. Die erworbenen Farbsehstörungen sind überwiegend zumindest am Anfang einseitig und oft von einer Visusreduktion begleitet. Sie werden durch Trübungen der optischen Medien, Erhöhung des Intraokulardruckes (M. Mantyjarvi \& M. Terasvirta, 1992), Erkrankungen der Retina und des Nervus opticus, Schädigung der Retina durch internistische Vorerkrankungen, altersbedingte Veränderungen sowie durch Intoxikationen hervorgerufen. 
Die häufigsten Ursachen sind Intoxikationen durch Medikamente oder durch Chemikalien am Arbeitsplatz, z. B. bei Lösungsmittelexposition beim Lackieren von Autos (Bockelmann et al., 2003). In vielen Fällen kommt eine Blau-GelbStörung vor (Iregren et al., 2002). Es gibt zahlreiche Medikamente, welche eine Farbsehstörung verursachen können. Die hier relevanten Medikamentengruppen sind vor allem Antibiotika, Antirheumatika, Schwermetalle, Schmerzmittel, Tuberkulostatika und Phenotiazine (Jaeger, 1977; Li et al., 2008). Die Einnahme von Digoxin führte bei älteren Patienten bei $20-30 \%$ zu einer Rot-Grün-Störung und bei 20\% zu einer Tritanstörung (Lawrenson et al., 2002). Ethamboutol durch die Neurotoxizität und Carbamazepin oder Diphenylhydantoin durch die Reduktion der Aktivität der Inhibitionsrolle der Stäbchen und Zapfen verursachen oft Farbsehstörungen (Bayer et al., 1995; Vistamehr et al., 2007). Auch das Medikament Sildenafil citrat („Viagra“) führt zu einer transienten Tritanopie (Jagle et al., 2004). Auch Alkoholkonsum kann eine Blaustörung hervorrufen (Zrenner et al., 1986). Raucher, die über 20 Zigaretten pro Tag konsumieren, können verschiedene Farbsehstörungen haben (Bimler et al., 2004; Erb et al., 1999).

Viele Erkrankungen der Retina, wie die Stargardtsche Erkrankung (M. Mantyjarvi \& K. Tuppurainen, 1992), die vitelliforme Makuladegeneration (Zrenner, 1985) und die seröse Retinopathie (Maaranen et al., 2000) sowie retinale Erkrankungen durch Einwirkung von Sonnenlicht (Kaluiny et al., 2006) führen oft zu einer Störung des Farbsehens. Auch Erkrankungen des Nervus opticus, wie die Neuritis nervi optici, bei der eine Rot-Grün-Störung (Zrenner, 1985), und die autosomal dominante infatile Optikusatrophie, bei der meistens eine Tritanstörung vorliegt, gehören zu den erworbenen Farbsehstörungen (Jaeger, 1954; M. I. Mantyjarvi et al., 1992).

$\mathrm{Zu}$ den wichtigsten internistischen Vorerkrankungen, welche neben den systemischen Komplikationen auch zu Farbsehstörungen führen, gehört der Diabetes mellitus. Dabei kommt es in der Regel zu einer Blau-Gelb-Störung bzw. Blau-Grün-Störung. Die Farbsehstörung tritt vor der typischen klinischen Veränderung in der Retina auf (Fong et al., 1999; Greenstein et al., 1990; 
Kinnear et al., 1972; Kurtenbach et al., 2002; Kurtenbach et al., 1994; Merbs et al., 1992; Roy et al., 1986; Thompson et al., 1979). Andere Erkrankungen sind die arterielle Hypertonie (Atonov et al., 1970; Luthke et al., 2010; Trusov, 1972), die HIV-Infektion (Kozak et al., 1994; Sommerhalder et al., 1998), die Sichelzellanämie (Roy et al., 1987), die Neurosyphilis (Behrens-Baumann et al., 1981) und Morbus Parkinson (Birch et al., 1998) sowie die Hämophilie (Jaeger et al., 1976).

Auch zerebrale Prozesse können nicht nur zur Visusreduktion und zu Gesichtsfelddefekten, sondern auch zu Störungen der Farbwahrnehmung führen (Pearlman et al., 1978; Rizzo et al., 1993; Vola et al., 1972).

\subsection{Grundlagen des Farbsehens}

Es gibt zwei vorherrschende Theorien, die das Farbsehen beschreiben:

Zum einen die Young-Helmholtz-Dreifarbentheorie (Helmholtz, 1867; Young, 1802). Es wurde postuliert, dass drei verschiedene Übertragungskanäle für die Farbinformation im visuellen System des Menschen vorliegen. Diese sind die drei Zapfenarten, die für Rot, Grün und Violett empfindlich sind. In der modernen Zeit konnte tatsächlich bestätigt werden, dass es drei verschiedene Fotopigmente gibt, welche spektralphotometrisch differenziert werden können: der kurzwellige Zapfen (S-Zapfen) mit dem Photopigment Cyanlab, der mittelwellige Zapfen (MZapfen) mit dem Photopigment Chlorolab und der langwellige Zapfen (L-Zapfen) mit dem Photopigment Erythrolab. So kann jeder wahrnehmbare Farbeindruck aus dem Empfindungsmaximum der entsprechenden Spektralfarben gemischt werden.

Zum anderen die Hering-Gegenfarbentheorie (Hering, 1874). Hering postulierte eine antagonistische Verarbeitung der Farbinformation auf retinaler Ebene. Er berichtete über drei antagonistische Kanäle: Grün und Rot, Blau und Gelb, Weiß und Schwarz. Seine Theorie konnte später durch die postrezeptorale Farbsignalverarbeitung auf Ebene der Ganglienzellen Bestätigung finden. Es ist 
heute bekannt, dass die Ganglienzellen in zwei Systeme eingeteilt werden können. Das ist zum einen das tonische oder parvozelluläre System, welches überwiegend ein farbantagonistisches Verhalten im Sinne von Blau/Gelb bzw. Rot/Grün zeigt. Daneben steht das phasische oder magnozelluläre System, welches überwiegend die Helligkeitsinformation verarbeitet.

Die beiden oben genannten Theorien ergänzen sich gegenseitig und können Aspekte der Farbverarbeitung gut und korrekt beschreiben.

\subsection{Definition der Farbe}

„Farbe ist diejenige Gesichtsempfindung eines dem Auge des Menschen strukturlos erscheinenden Teiles des Gesichtsfeldes, durch die sich dieser Teil bei einäugiger Beobachtung mit unbewegtem Auge von einem gleichzeitig gesehenen, ebenfalls strukturlosen angrenzenden Bezirk allein unterscheiden kann." (DIN, 2009)

Farbe ist komplex, weil sie nicht nur von der Wellenlänge abhängig ist (Kaiser \& Boyton, 1996). Zur Beschreibung der Farbe sind im Prinzip drei Koordinaten erforderlich, welche von Psychologen im 19. Jahrhundert beschrieben wurden.

Farbton: Dieser wird durch die Wellenlänge bestimmt im Sinne von Rot, Grün, Gelb, Blau. Im Bild geht das Blau ins Violett über, da der Rotanteil der Farbe steigt (Abb. 10).

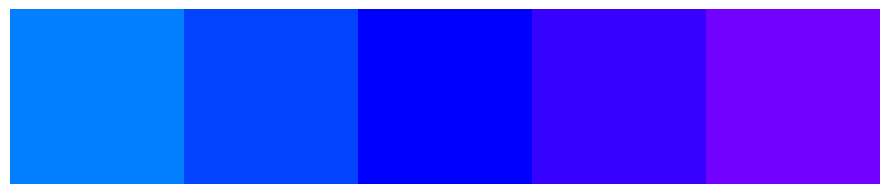

Abb. 10: Unterschiedliche Farbtöne von Blau

Sättigung: Dies ist die Farbmischung des Farbtons mit Weiß, Grau oder Schwarz. Je farbiger bzw. reiner eine Farbe ist, desto stärker ist sie gesättigt. Je geringer die Sättigung einer Farbe ist, desto farbloser und unauffälliger wirkt sie (Abb. 11). 


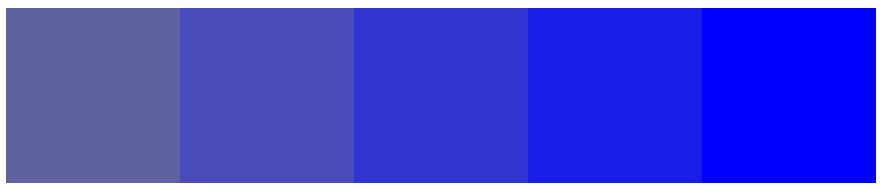

Abb. 11: Unterschiedliche Sättigung

Helligkeit: Damit sind die verschiedenen Grautöne gemeint, auch jede Farbe kann als heller oder dunkler empfunden werden (Gouras, 1995; Greve, 20052010) (Abb. 12).

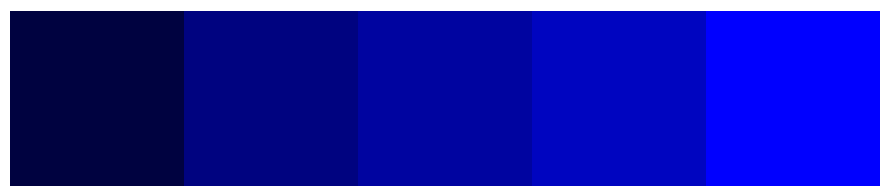

Abb. 12: Unterschiedliche Helligkeit

\subsection{Farbsysteme}

Über die Jahre sind mehr als 60 verschiedene Farbordnungen und Farbmodelle entwickelt worden. Ein Farbsystem ist eine Systematik zur Anordnung der Farbvalenzen, die auf unterschiedliche Art Farben erzeugen. Dies ist möglich durch „Mischung“ von Licht als Lichtfarben oder die Verwendung von Farbmitteln auf einem Trägermaterial als Körperfarben. Je nach Anwendungszweck kann eine unterschiedliche Anzahl von Grundfarben genutzt werden: Mindestens drei, aber auch vier oder mehr farbgebende Substanzen werden verwendet. Diese sind allerdings nicht mehr unabhängig voneinander.

Das Farbmodell entsteht aus dem abstrakten Farbsystem meist dreidimensional für die praktische Darstellung, die unterschiedlicher Form sein kann. Innerhalb der Modelle sind allen Farben eindeutige Zahlenwerte, die Farborte, zugeordnet (Wikipedia, 2015b). Die gebräuchlichsten Farbsysteme werden im Folgenden beschrieben.

\subsubsection{CIE-Normfarbdreieck}

Das CIE-Normvalenzsystem oder CIE-Normfarbsystem wurde von der Internationalen Beleuchtungskommission 1931 (CIE - Commission internationale 
de l'éclairage) definiert, um eine Relation zwischen der menschlichen Farbwahrnehmung (Farbe) und den physikalischen Ursachen des Farbreizes (Farbvalenz) herzustellen (Adams et al., 1975).

Bei dem 1931 entwickelten CIE-Normfarbsystem blickt der gemittelte Beobachter auf eine Fläche mit einem Sichtfeld von $2^{\circ}$ mittig zur Hauptrichtung, wobei sich das bei dem 1964 entwickelten CIE-Farbsystem auf das 10-Sichtfeld des Menschen bezieht.

Im Verlauf wurde die zweidimensionale CIE-Normfarbtafel entwickelt, um den vom Betrachter wahrgenommenen dreidimensionalen Farbraum übersichtlicher darstellen zu können. Dabei wird die dritte Komponente z für jeden Punkt der Farbtafel rechnerisch aus den beiden anderen durch die Beziehung $x+y+z=1$ ermittelt. Die hufeisenförmige Fläche möglicher Farben ist bei der CIENormfarbtafel auf einem Koordinatensystem aufgetragen, auf dem $\mathrm{x}$ - und y-Anteil (der ClE-genormten theoretischen Grundfarben X (Rot), Y (Grün) und Z (Blau)) einer beliebigen Farbe $P$ direkt abgelesen werden können. Durch die Grundbedingung $x+y+z=1$ lässt sich der $z$-Anteil jeweils rechnerisch $(z=1$ $x-y)$ ermitteln. Die Gesamtheit möglicher Farben wird durch die das Hufeisen umgrenzende Spektralfarblinie (spektral reine Farben) sowie die untere Purpurlinie eingefasst (Wikipedia 2015).

Zentraler Bezugspunkt der Tafel ist der in jeder Farbmesssituation wesentliche Weißpunkt W. Der im Diagramm mit W gekennzeichnete Punkt ist derjenige theoretische Weißpunkt, der alle drei Farben zu je 1/3 ( $x, y$ und $z=0,333$ ) repräsentiert. Abhängig von der Beleuchtungssituation kann sich der Weißpunkt praktisch überall innerhalb des Hufeisens befinden. Technisch von Bedeutung ist nur die Black-Body-Kurve. Die Farben sind auf deren Verlauf als Temperatur eines idealen Strahlers (schwarzer Körper) in Kelvin angegeben. Ausgehend vom Weißpunkt können alle als farbtongleich empfundenen Farben auf einer Linie durch den Punkt $\mathrm{P}$ abgelesen werden. Auf der genau gegenüberliegenden Seite von W können die Komplementärfarben auf der verlängerten Linie W-Q abgelesen werden. Der Punkt Q', der in diesem Fall durch den Schnitt mit der 
Purpurlinie definiert wird, stellt dabei die äußerste (reinste) Komplementärfarbe dar (Wikipedia, 2010) (Abb. 13).

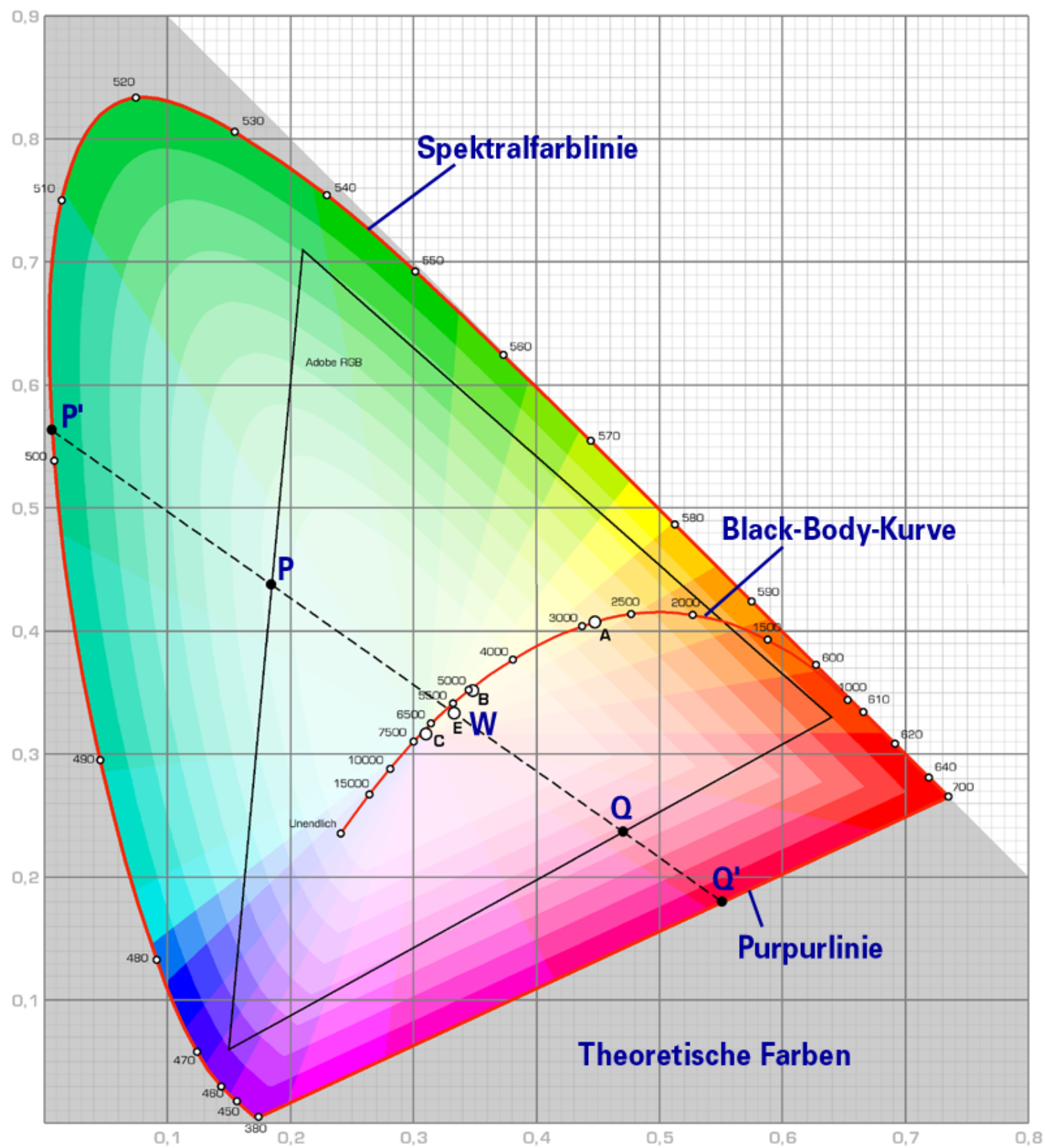

Abb. 13: CIE-Normfarbdreieck (Wikipedia, 2010)

\section{Verwechselungsfarben/Verwechselungslinien}

Fehlt ein Zapfentyp, werden diejenigen Farben verwechselt, die sich in den Signalen dieses Zapfentyps unterscheiden. Solche Farben liegen auf den Protan, Deutan- oder Tritan-Verwechselungslinien. Unterwertigkeit eines Zapfentyps 
verringert die Farbunterschiedsempfindlichkeit entlang seiner Verwechselungslinien.

Die Verwechselungslinien verbinden alle Punkte, welche die verbleibenden beiden Rezeptorentypen identisch reizen und treffen sich in jeweils einem Kardinalpunkt. Der Kardinalpunkt entspricht der „virtuellen Farbe“, an der die beiden jeweils anderen, normalen Zapfen gar nicht aktiviert werden.

Die Farben der Bildpunkte von Sehzeichen und Umfeld einer pseudoisochromatischen Tafel sind verschiedenen Abschnitten je einer Verwechselungslinie entnommen. Für die Erfassung von Protan-, Deutan-, Tritan- und Tetartanstörungen werden deshalb verschiedene Tafeln benötigt, bei denen es außer auf die Richtung der Verwechselungen im Farbdreieck auch auf die Distanz der Farborte und auf die Helligkeiten ankommt.

Bei Farbflecktests stellt der Patient seine eigenen Verwechselungslinien dar, indem er die Farbmarken schrittweise in der Reihenfolge seiner Wahrnehmung anordnet (Krastel et al., 2009) (Abb. 14).

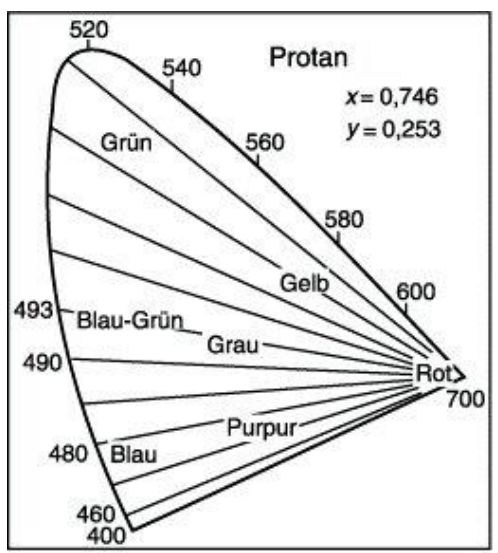

a

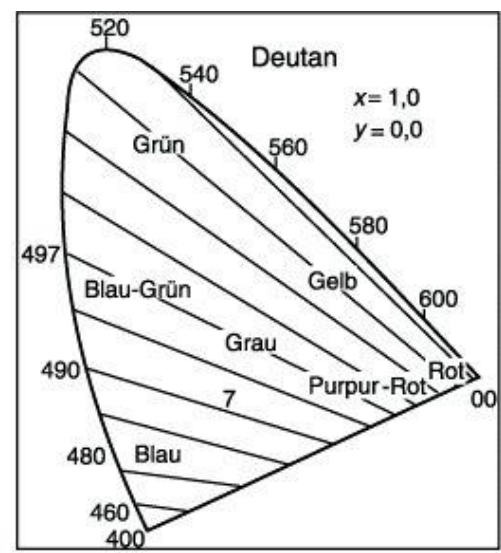

b

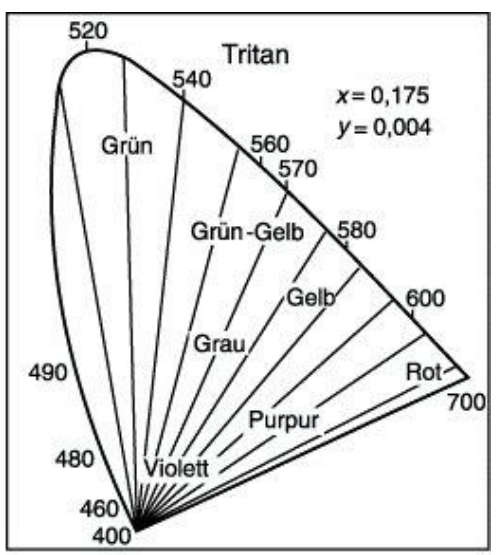

Abb. 14: Farbverwechselungsgeraden des Protanopen (a), Deuteranopen (b) und Tritanopen (c) (Spektrum.de, 2015). 


\subsubsection{Munsell-Farbraum}

Das Munsell-Farbsystem (Munsell Color System oder Munsell Color Order System) wurde von dem Künstler Albert Henry Munsell (1858-1918) zwischen 1898 und 1905 veröffentlicht. Munsells Farbatlas wurde durch die Optical Society of America 1929 nachgemessen und -kalibriert.

Das Munsell-System (Abb. 15) ist eines der ersten vollständigen, am weitesten verbreiteten und heute noch genutzten Farbsysteme. Es ist vor allem in den USA und in Japan weitverbreitet. Dieses System ist unabhängig von der Beleuchtung und der Größe der betrachteten Farbfläche. Die drei Ordnungsprinzipien sind Hue (Farbton), Chroma (Sättigung) und Value (Wertigkeit, Helligkeit). Priorität dieses Systems ist der Farbton.

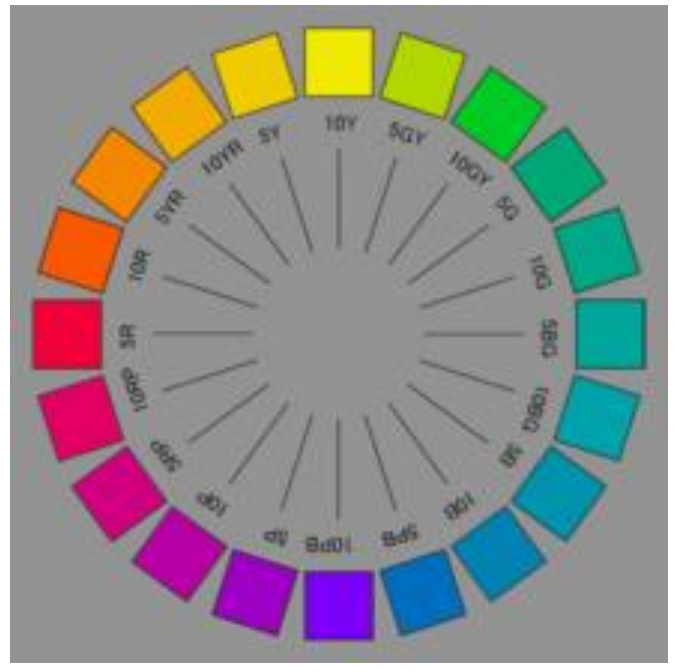

Abb. 15: Munsell-Farbsystem (Wilkipedia, 2015)

Hue: Hue steht für die Wellenlänge oder die dominante Wellenlänge. Es gibt fünf Hauptbunttöne: Rot $(R)$, Gelb $(Y)$, Grün $(G)$, Blau $(B)$, Purpur (P) und fünf weitere Zwischenbunttöne: YR (Gelb-Rot), GY (Grün-Gelb), BG (Blau-Grün), PB (Purpur-Blau), RP (Rot-Purpur). Diese Aufstellung ergibt eine 10-teilige Farbfläche. Diese zehn Farbtöne werden abermals in zehn Abstufungen unterteilt. Für die Kennzeichnung der Bunttöne werden zu den 
Buchstabensymbolen noch Zahlen (0 bis 10 ) zugesetzt: So werden die Farbtöne nach dem Muster 1RP, 5P, 3Y oder auch 7,5 GY, 2,5 PB bezeichnet.

Chroma: Chroma ist ein Munsell-Begriff für die Sättigung. Chroma wird in einer Skala von 0 bis zu verschiedenen Maxima angezeigt. Die Distanz vom Mittelpunkt zum Farbpunkt (zum Rand hin) ergibt eine zunehmende Sättigung, ohne Änderung von Helligkeit. Entsprechend der (visuellen) Konstruktion bleibt der Farbton erhalten und ändert sich nicht.

Value: Value wird in einer Zahlenskala von 1 (Schwarz) bis 10 angegeben (Friendly et al.). Diese Skala repräsentiert die Reflexion und die Helligkeit.

Zur Benennung erhält jedes Farbmuster im Munsell-System, also jede dargestellte Farbe ein Farbzeichen der Form $\mathrm{H}$ V/C, wobei $\mathrm{H}$ (Hue) für Buntton, V (Value) für Helligkeit und C (Chroma) für Sättigung steht (Abb. 16).

Beispielhaft sei die Farbe 2,5 YR 5/10 beschrieben, ein Gelb-Rot, welches zum Rot tendiert, eine mittlere Helligkeit hat und maximal gesättigt erscheint (Gouras, 1991; Wilkipedia, 2015).

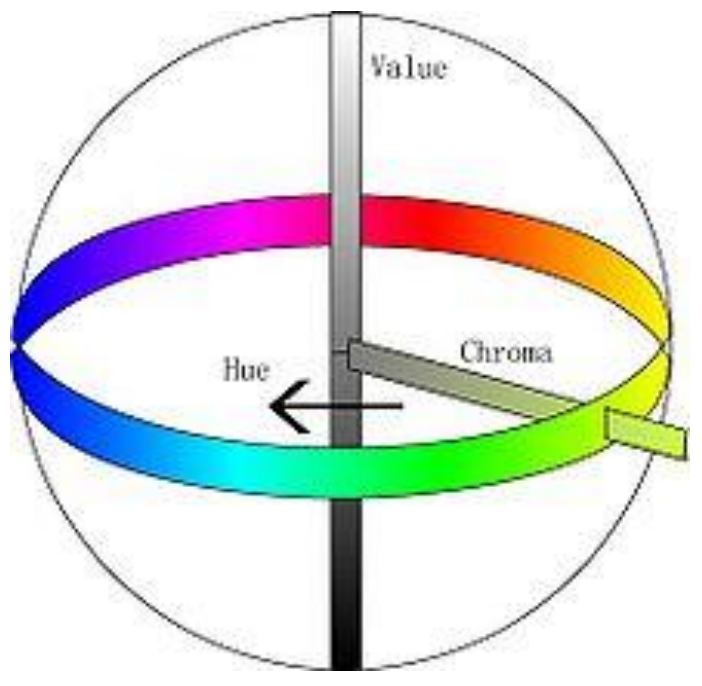

Abb. 16: Munsell-Farbsystem

Die rasche Entwicklung der Fernseh- und Computer- sowie Drucktechnik führte auch zur Entwicklung von geräteabhängigen Farbmodellen, wie des RGB- und des CMY/CMYK-Farbmodells (Gierling, 2001). 


\subsubsection{RGB-Farbmodell}

Es handelt sich hierbei um ein Farbkoordinatensystem mit drei Primärfarben: $R$ (Rot), G (Grün) und B (Blau). Jede Primärfarbe kann einen Intensitätswert zwischen 0 (aus/am niedrigsten) und 1 (ein/am höchsten) aufweisen. Wenn diese Primärfarben in unterschiedlichen Intensitätsgraden gemischt werden, entsteht eine Vielzahl von Farben. Die Palette dieser Farben bildet den würfelförmigen RGB-Farbraum. Die Ecke des RGB-Farbwürfels, die sich im Ursprung des Koordinatensystems befindet, entspricht der Farbe Schwarz, während die dem Ursprung diagonal gegenüberliegende Würfelecke der Farbe Weiß entspricht. Die diagonale Linie zwischen Schwarz und Weiß entspricht allen Grautönen zwischen Schwarz und Weiß und wird als Grauachse bezeichnet. Jede beliebige Farbe innerhalb des Würfels kann anhand ihrer Koordinaten festgelegt werden: $(r, g, b)$, z. B. $(0,0,0)$ für Schwarz, $(1,1,1)$ für Weiß, $(1,1,0)$ für Gelb usw. Bei der Farbspezifikation nach dem RGB-Modell handelt es sich um einen additiven Prozess. Man beginnt mit Schwarz und fügt die entsprechenden Primärkomponenten hinzu, um eine gewünschte Farbe zu erzeugen (Zhigang Xiang, 2000) (Abb. 17).

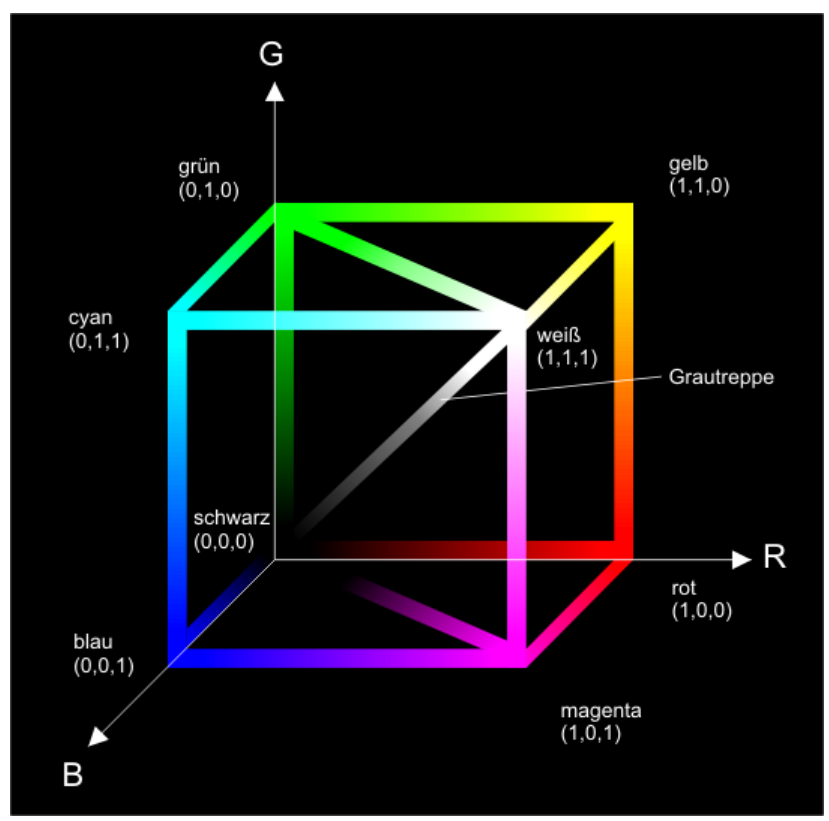

Abb. 17: RGB-Farbsystem (Computerartikel-Archiv, 2009) 
Der Adobe-RGB-(1998)-Farbraum ist eine besondere Form des RGB-Farbraums mit deutlichen Verbesserungen bei den Türkis- und Grün-Tönen (Wikipedia, 2015d).

\subsubsection{CMY/CMYK-Farbmodell}

Das CMY-Farbmodell ist ein Komplementärfarbenmodell. Die Farben werden mithilfe eines subtraktiven Prozesses definiert. Man beginnt mit Weiß und entfernt die entsprechende Primärkomponente, um die gewünschte Farbe zu erzeugen. Wenn zum Beispiel Rot von Weiß subtrahiert wird, besteht die verbleibende Farbe aus Grün und Blau und wird als Cyan bezeichnet. Die Primärfarben sind C (Cyan), M (Magenta) und $Y$ (Yellow, Gelb). Die am Punkt $(0,0,0)$ befindliche Ecke des CMY-Farbwürfels entspricht Weiß, während die Würfelecke am Punkt $(1,1,1)$ Schwarz darstellt (Abb. 18).

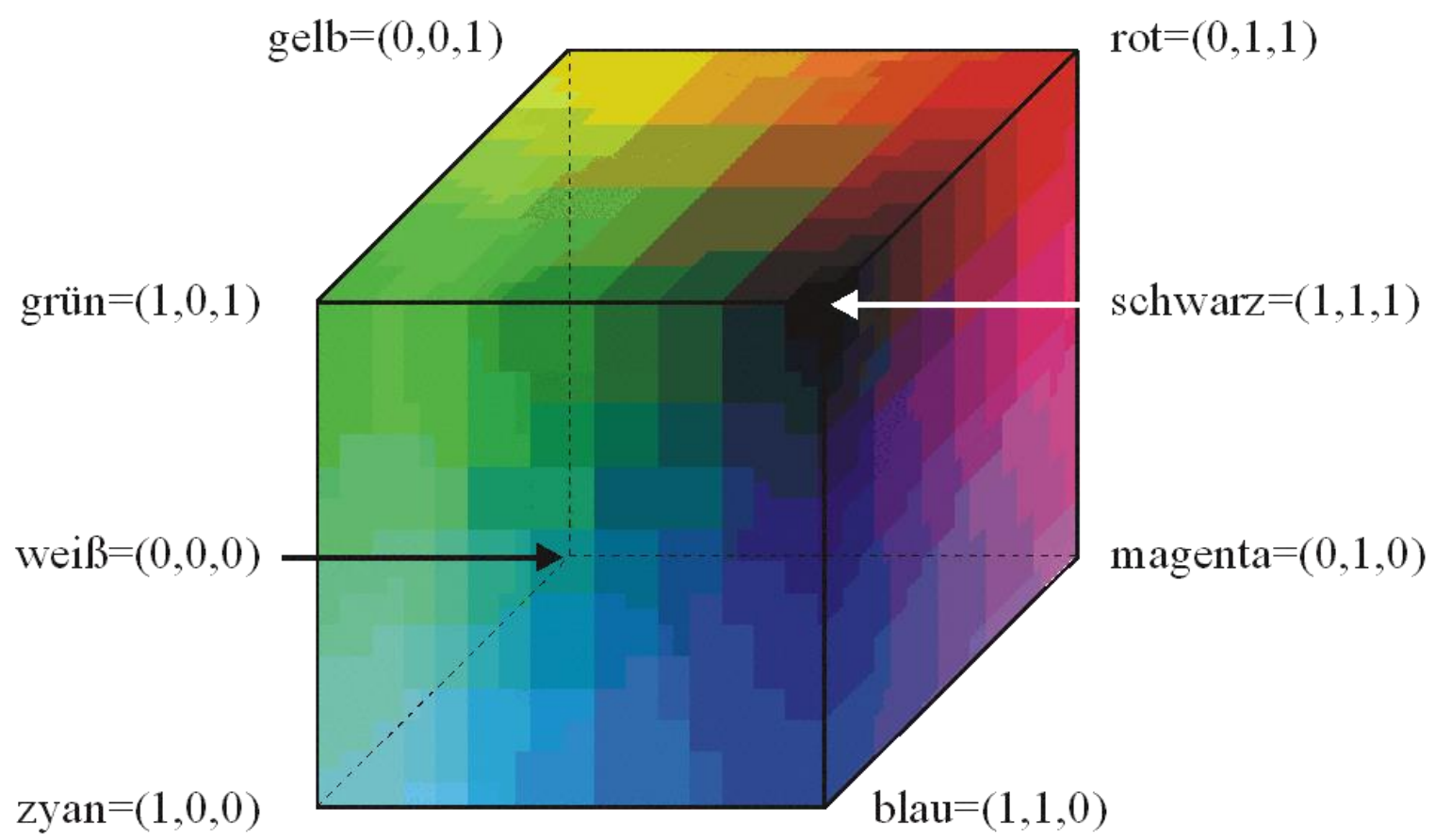

Abb. 18: CMY-Farbmodell (Dortmund, 1999) 


\subsection{Untersuchungsmethoden bei Farbsehstörungen}

Die Untersuchung des Farbsehens kann mit verschiedenen Methoden durchgeführt werden. Man kann mittels pseudoisochromatischer Tafeln, Farbfleck- oder Farbordnungstests, Verfahren mit Lichtreizen breiter spektraler Bandcharakteristik, Farbtests mit spektral schmalbandigen Lichtreizen, Leuchtdiodentests, Bildschirmverfahren, Anomaloskop sowie Farbperimeter eine Farbsehstörung detektieren (Krastel et al., 2009).

\subsubsection{Pseudoisochromatische Tafeln}

Zum Screening zu angeborenen Farbsehstörungen werden die pseudoisochromatischen Farbtafeln verwendet. Bei diesen Tafeln gibt es vier verschiedene Typen (Gouras, 1991):

a. Transformationstafeln: Bei diesen Tafeln nimmt eine Person ohne Farbsehstörung ein Zeichen wahr, und bei Vorliegen einer Farbsehstörung wird ein anderes Zeichen wahrgenommen (Abb. 19).

b. Tafeln mit verschwindenden Zeichen: Bei diesen Tafeln nimmt eine Person ohne Farbsehstörung ein Zeichen und eine Person mit Farbsehstörung kein Zeichen wahr (Abb. 20).

c. Tafeln mit versteckten Zeichen: Bei diesen Tafeln nimmt eine Person ohne Farbsehstörung keine Figur und eine Person mit Farbsehstörung ein Zeichen wahr (Abb. 21).

d. Diagnostische Tafeln: Ein Zeichen wird besser als das andere von Personen mit Farbsehstörungen wahrgenommen (Abb. 22). 


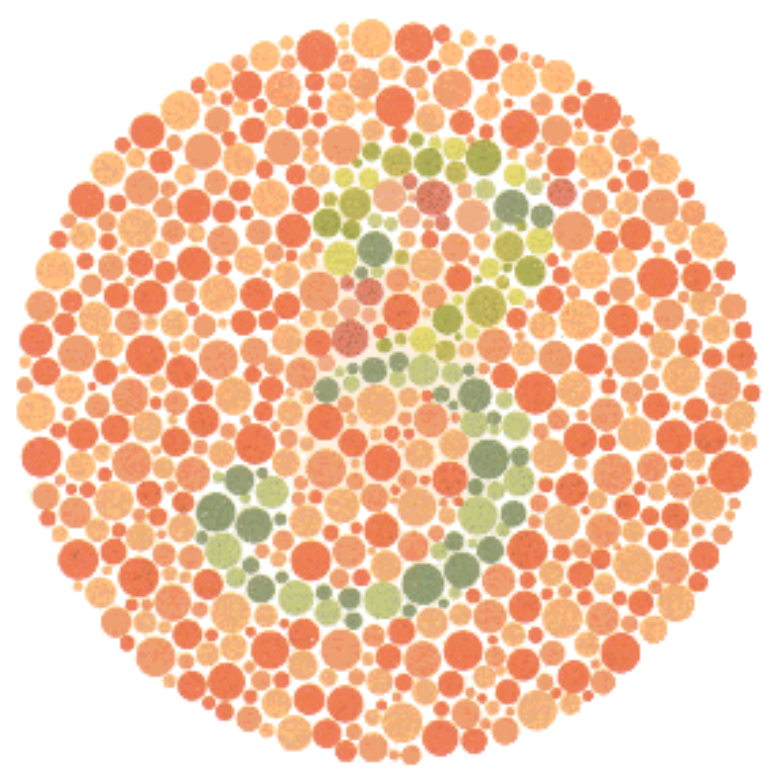

Abb. 19: Beispiel einer Transformationstafel (Farbnormale sollten die Nummer 3 lesen können. Rot-Grün-Fehlsichtige sehen die Nummer 5).

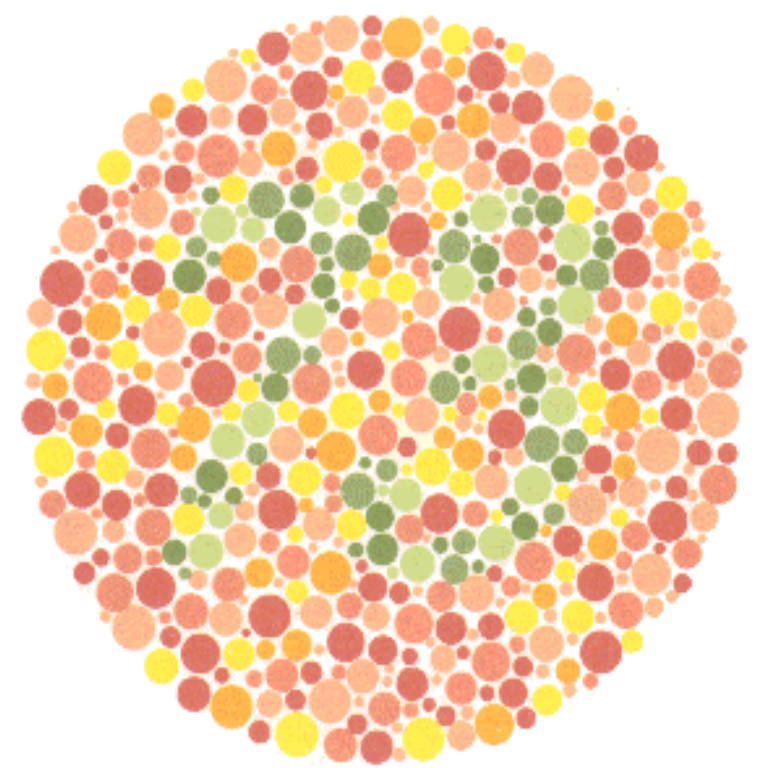

Abb. 20: Beispiel einer Tafel mit verschwindenden Zeichen (Farbnormale lesen die Nummer 73. Die Mehrzahl der Rot-Grün-Farbfehlsichtigen oder Farbenblinden erkennen die 73 nicht oder sehen eine andere Zahl). 


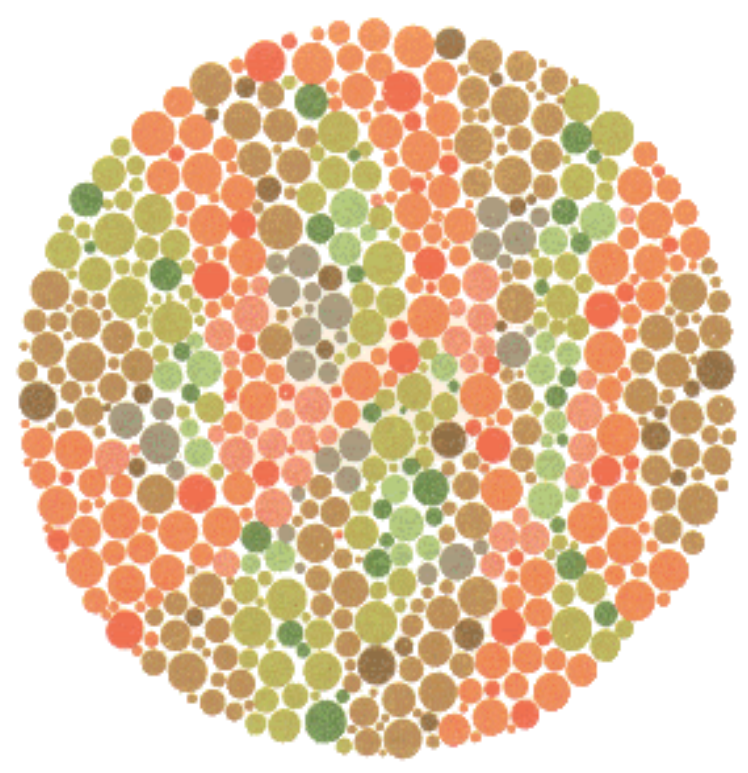

Abb. 21: Beispiel einer Tafel mit versteckten Zeichen (Farbnormale und total Farbenblinde können kein Zeichen erkennen. Die Mehrheit der Rot-Grün-Fehlsichtigen erkennt hier die Zahl 45).

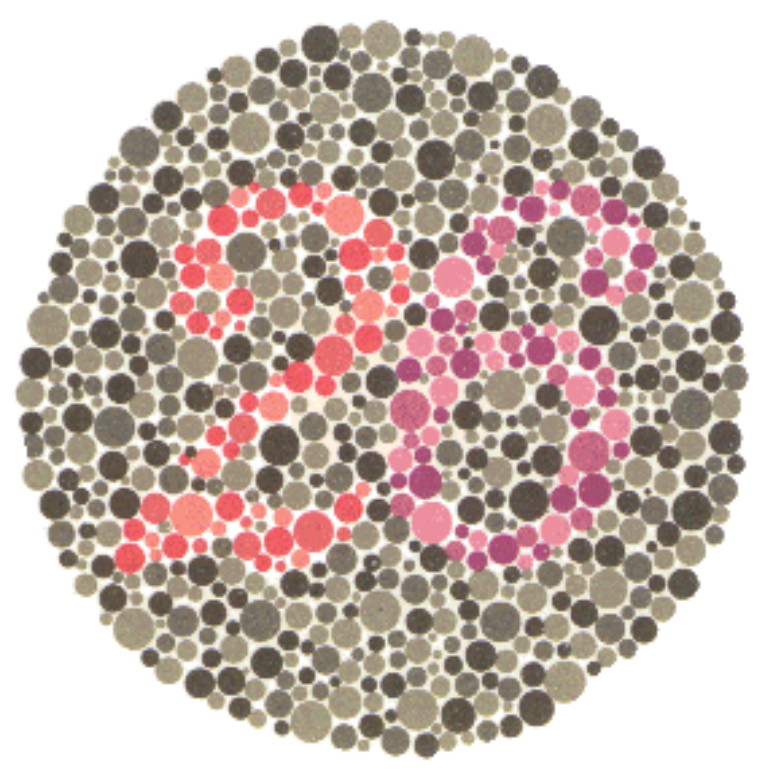

Abb. 22: Beispiel einer diagnostischen Farbtafel (Farbnormale sollten die Zahl 26 erkennen. Von Protanopen und stark Protanomalen wird eine 6 gesehen. Gering Protanomale sehen sowohl die 2 als auch die 6, wobei die 6 deutlicher wahrgenommen wird. Deuteranope und stark Deuteranomale können nur die Ziffer 2 lesen. Im Falle einer schwachen Deuteranomalie wird wieder die Zahl 26 erkannt, aber hier wird die Zahl 2 deutlicher wahrgenommen als die 6 (Rand et al., 1956)). 
Der Proband hat sich zunächst mit dem Charakter der Optotypen vertraut zu machen. Daher wird eine Demonstrationstafel vorgegeben, welche auch bei Vorliegen einer Farbsehstörung gelesen werden kann (Krastel et al., 2009). Für Rot-Grün-Störungen sind die Ishihara-Tafeln (Nakajima et al., 1960), die Velhagen/Broschmann/Kuchenbecker-Tafeln, die Standard Pseudoisochromatic Plates I (Hovis et al., 1996), die American Optical Company Plates nach Hardy, Rand und Rittler (Rand et al., 1956), der City University Color Vision Test (Birch, 1997a) und der Neitz Color Vision Worksheet Test geeignet. Für Blausinnstörungen sind die Velhagen/Broschmann/Kuchenbecker-Tafeln (Broschmann, 2009; Velhagen et al., 1995, 1997, 2000), die Standard Pseudoisochromatic Plates (SPP) II (Pinckers et al., 1985), die American Optical Company Plates nach Hardy, Rand und Rittler (AO HRR) sowie das Lanthony Tritan Album geeignet (Lanthony, 1986; Zrenner, 1987).

Für Kinder gibt es den Matsubara-Kojima-Kinder-Farbtafeltest, der ab dem 3. Lebensjahr verwendet werden kann (Krastel, 1995).

Die Untersuchung des Farbsehens mittels pseudoisochromatischer Tafeln sollte immer unter standardisierten Untersuchungsbedingungen erfolgen. Die Testung des Farbsehens mittels Velhagen/Broschmann/Kuchenbecker-Tafeln sollte in 70 $\mathrm{cm}$ Abstand für $15 \mathrm{sec}$ unter Tageslicht erfolgen. Bei den Ishihara-Tafeln ist ein Untersuchungsabstand von $75 \mathrm{~cm}$ nötig. Anstelle des Tageslichts sind auch Leuchtröhren mit kontinuierlicher, tageslichtähnlicher Emission für Farbmusterungszwecke, z. B. Philips TL 54, TL 55, TL 57 oder Osram Biolux geeignet. Die Farbtemperatur soll über $5000^{\circ} \mathrm{K}$ bis $6800^{\circ} \mathrm{K}$ liegen. Die Tafeln sind in etwa rechtem Winkel zur Blickrichtung vorzulegen. Die vom Hersteller empfohlene Beobachtungszeit beträgt für die Ishihara-Tafeln drei Sekunden und für die Velhagen/Broschmann/Kuchenbecker-Tafeln 15 Sekunden (Krastel, 2007; Krastel et al., 2009). 


\section{Velhagen/Broschman/Kuchenbecker-Tafeln}

Ursprünglich sind die Farbtafeln von Stilling entwickelt worden. Ab der 15. Auflage sind diese von G. Weil, von 1929 bis 1939 von E. Hertel (18. bis 20. Auflage), von 1952 bis 1974 von Velhagen (21. bis 25. Auflage) und ab 1985 (27. Auflage) von Broschmann und seit 2011 (34.Auflage) von Kuchenbecker aktualisiert und herausgegeben worden. Die Sensitivität und Spezifität der einzelnen Auflagen ist unterschiedlich, sodass einige Auflagen in visuell auffälliger Weise voneinander abweichen können. Daher sollte die benutzte Auflage immer angegeben werden. Die Tafeln erscheinen im Georg Thieme Verlag (Broschmann, 2009; Heinsius, 1973; Krastel, 1995; Stilling, 1877).

\section{$\underline{\text { Ishihara-Tafeln }}$}

Der Ishihara-Test ist der meist verbreitete Screening-Test zur Detektion einer Rot-Grün-Farbsehstörung (Birch, 1997b). Der Test wurde erstmals 1906 veröffentlicht und war der erste kommerziell hergestellte Test (Dain, 2004). Es hat sich gezeigt, dass es von Auflage zu Auflage sowie mit der Alterung der pseudoisochromatischen Tafeln zu Unterschieden in der Helligkeit, im Farbton und in der Sättigung kommt (Hyon et al., 2005).

\subsubsection{Farbordnungstests}

Die Farbordnungstests dienen zur Detektion von erworbenen Farbsehstörungen. Es werden kleine Farbtöpfchen dargeboten, und der Betrachter wird aufgefordert, die Farben in einer seiner Wahrnehmung entsprechenden Reihenfolge zu sortieren. Damit kann man auch eine angeborene von einer erworbenen Störung differenzieren. Bei den angeborenen Farbsehstörungen zeigen sich parallele Verwechselungsgeraden, wobei es bei erworbenen Störungen häufig zu einer erratischen multidirektionalen Anordnung von Verwechselungsgeraden kommt.

Die gebräuchlichsten Farbordnungstests sind:

- der Panel-D-15-Test in der saturierten und desaturierten Form (nach Farnsworth und nach Lanthony (Abb. 23) 
- der Farnsworth-Munsell-100-Hue-Test (Abb. 24)

- der 28-Hue-Test nach Roth (Lachenmayr B., 2006).

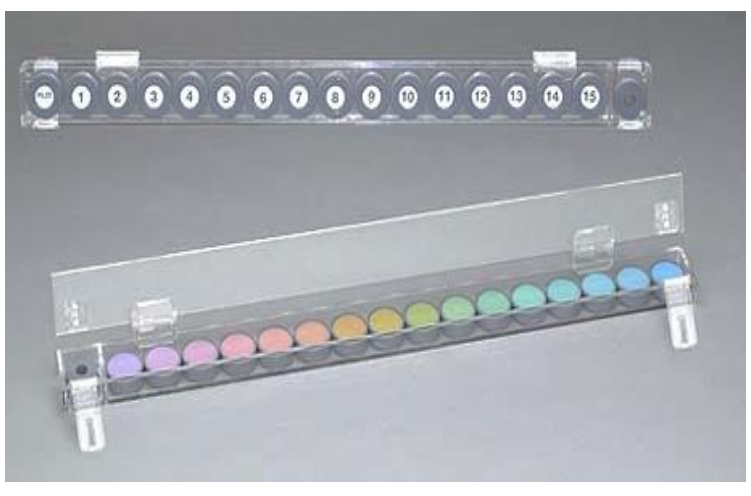

Abb. 23: Panel D-15-Test ("Colour vision test," 2004)

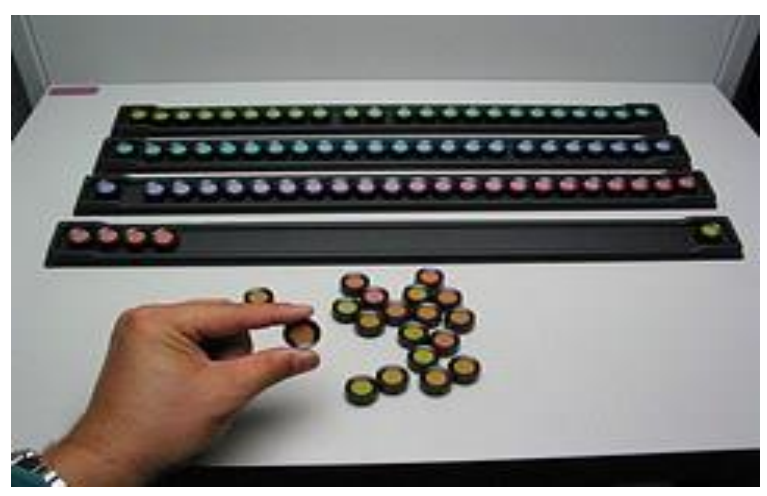

Abb. 24: Der Farnsworth-Munsell-100-Hue-Test (Aguilera, 2010)

Dazu gibt es die folgenden Tests, die seltener verwendet werden:

- City-University-Test nach Fletcher

- Farbtestscheibe nach Guilino und Ledl (Krastel et al., 2009).

Die Farbflecktests sind in der Erfassung von Rot-Grün-Störungen weniger sensitiv als Farbtafeln. Die Prüfmarken sind bei Farbflecktests größer, als die spatiale Auflösung des Rot-Grünsinns es erfordert. Farbflecktests sind besonders für die Untersuchung des Blausinns, weniger des Rot-Grün-Sinns geeignet, weil das kurzwellige System in der Netzhaut weitmaschiger als das Rot-Grün-System ist (Krastel, 2007). 


\subsubsection{Anomaloskope}

\section{$\underline{\text { HMC-Anomaloskop }}$}

Das HMC-Anomaloskop ermöglicht die qualitative und quantitative Diagnostik von angeborenen sowie erworbenen Farbsehstörungen. Durch verschiedene Gleichungen ist die Untersuchung des Rot-Grün-Sinns und des Blausinns möglich.

Die Rayleigh-Gleichung dient zur Untersuchung des Rot-Grün-Sinns:

Grün $(549 n m)+$ Rot $(666 n m)=$ Gelb $(589$ nm).

Die Moreland-Gleichung eignet sich zur Untersuchung des Blausinns:

Blau $(436 n m)+$ Grün $(490 n m)=$ Zyan $(480 \mathrm{~nm})+$ Gelb $(589 \mathrm{~nm})$.

Das verwendete Prinzip ist die additive Farbenmischung und die Metamerie. Es wird dem Beobachter ein kreisförmiges Prüffeld vorgegeben. In der oberen Hälfte überlagern sich zwei spektrale Farbreize. In der unteren Hälfte wird ein spektraler Farbreiz angeboten, welcher der Farbmischung der oberen Hälfte gleicht. Das Mischungsverhältnis des Mischlichtes oben sowie die Helligkeit des unteren Teils werden variiert, bis subjektiv beide Teile als gleich empfunden werden (Abb. 25).

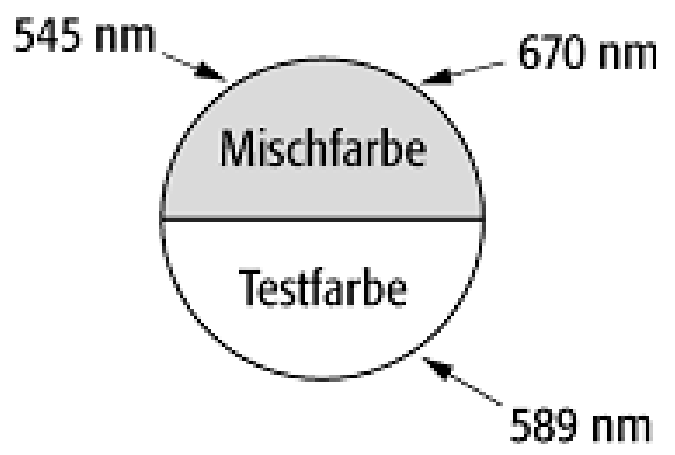

Abb. 25: Kreisförmiges Prüffeld des HMC-Anomaloskops (Spektrum, 2015)

Die Rayleigh-Gleichung

In dem horizontal geteilten Prüffeld befinden sich das Mischfeld aus Grün und Rot oben und das Vergleichsfeld mit Gelb unten. Das Mischfeld kann von 0 bis 73 eingestellt werden. 73 bedeutet eine grünfreie Einstellung des Mischfeldes. 
Die Einstellung bei den Normalsichtigen beträgt 34 bis 46 Skalenwerte. Als Mittelnormgleichung ist der Mittelwert 40/15 festgelegt. In diesem Fall beträgt der Anomalquotient 1,0.

Nach dem Fachnormenausschuss Farbe sind Probanden von

0 bis $<0,7 \quad$ protanomal,

0,7 bis $1,4 \quad$ normal,

$>1,4$ bis unendlich deuteranomal

(Oculus).

\section{Nagelanomaloskop}

Das Nagelanomaloskop (Abb. 26) wurde 1907 von dem deutschen Opthalmologen und Physiologen Willibald A. Nagel (1870-1911) erfunden.

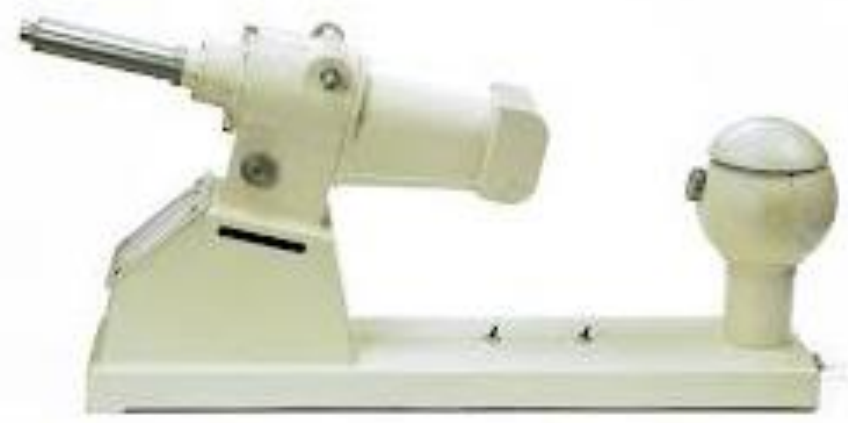

Abb. 26: Nagelanomaloskop

Die Untersuchung mit dem Nagelanomaloskop ist ein Hilfsmittel zur Differenzialdiagnose von angeborenen und erworbenen Farbsehstörungen.

Das Nagelanomaloskop hat ein Gesichtsfeld mit dem Durchmesser von $2^{\circ} 10^{\prime}$. In der unteren Hälfte wird ein Natriumgelb mit der Wellenlänge von 589,3 nm dargeboten, dessen Leuchtdichte zwischen 0 und einem Maximum verändert werden kann. In der oberen Hälfte wird entweder Quecksilbergrün von 546 nm oder Lithiumrot von 670,8 nm bzw. Mischungen dieser beiden Lichter geboten. Die Beobachtungszeit darf drei Sekunden nicht überschreiten. Dem Probanden 
werden fünf Gleichungen vorgelegt: die Mittelnormgleichung, die Deuteranomalen- und die Protanomalengleichung sowie die beiden Endgleichungen, d. h. reines Grün bzw. reines Rot im Vergleich mit Gelb. In jedem Fall sind als erstes etwaige Helligkeitsunterschiede durch Nachstellen der Gelbschraube durch den Untersucher zu beseitigen. Erst dann wird gefragt, ob die beiden Hälften des Gesichtsfeldes gleich- oder verschiedenfarbig sind.

Wird die Mittelnormgleichung angenommen (die beiden Halbfelder werden als halbfarbig erkannt), so es handelt sich beim Probanden entweder um einen normalen Trichromaten oder um einen Dichromaten bzw. vereinzelt um einen Fall von Extremanomalie. Wird die Mittelnorm verworfen (die beiden Halbfelder werden verschiedenfarbig gesehen), so lassen wir sie benennen, und zwar stets in der Reihenfolge obere Hälfte/untere Hälfte. Wenn die Antwort „rot/grün (oder gelb)“ lautet, so ist eine Deuteranomalie anzunehmen, bei der Angabe „grün/rot (oder gelb)" Protanomalie. Endgültig darf dies aber erst nach der Vorlage von im Allgemeinen vier weiteren Einstellungen und deren entsprechender, übereinstimmender Beantwortung diagnostiziert werden (F.Hollwich, 1977).

Weitere Anomaloskope sind das Neitz-Anomaloskop, das BKG-IF2-Anomaloskop und das Spectum Color Vision Meter (Krastel et al., 2009).

\subsection{Tablet-PC}

\subsubsection{Funktionsweise von Tablet-PCs}

Ein Tabletcomputer ist ein tragbarer, flacher Computer in besonders leichter Ausführung mit einem Touchscreen, aber, anders als bei Notebooks, ohne ausklappbare mechanische Tastatur. Ein Tablet-PC lässt sich durch die leichte Bauart und den berührungsempfindlichen Bildschirms leicht handhaben. Die Geräte ähneln in Leistungsumfang, Bedienung und Design modernen Smartphones und verwenden meist ursprünglich für Smartphones entwickelte Betriebssysteme (Wikipedia, 2015e). 
Tablet-PCs sind multimedial und besonders vielseitig. Zusätzlich zu klassischen Office-Programmen zum Verfassen von Texten, Erstellen von Tabellen etc. verfügen sie vor allem über unzählige Funktionen zur Unterhaltung.

Durch die zahlreichen Applikationen, die bereits im Tablet-PC integriert sind bzw. integriert werden können, lässt sich jederzeit gewicht- und platzsparend auf eine Vielzahl von Büchern und Zeitschriften zugreifen.

Aufgrund einer sehr guten Bildauflösung und auch eines Audio-Anschlusses für Kopfhörer sind Tablet-PCs zur Wiedergabe von Filmen und Videoclips geeignet. Durch das Touchscreen-Display sind die Tablet-PCs bedienfreundlich und besitzen eine hohe Reaktionsfähigkeit.

Bild- und Film-Aufnahmen sind über integrierte Kameras möglich und können mit der passenden Applikation auch bearbeitet werden. Eine zweite Kamera dient in erster Linie der Videotelefonie, ein weiteres Feature, das viele Tablet-PCs ermöglichen.

Die elementar wichtigste Funktion bleibt jedoch die Internetfähigkeit der TabletPCs. Diese ermöglicht die Multifunktionalität der Tablet-PCs, denn so können Bücher, Filme, Spiele etc. schnell und einfach bezogen werden. Auch die Kommunikation via E-Mail, Videotelefonie etc. ist möglich. Die Anzahl der Funktionen eines Tablet-PCs kann durch den Zugriff auf die dem jeweiligen Betriebssystem zugeordneten App-Stores beliebig erweitert werden (TabletPCs.eu, 2015b).

Der US-amerikanische Technologie-Hersteller Apple brachte den ersten seiner Tablet-PCs unter dem Namen iPad® 2010 auf den Markt (Abb. 27). 


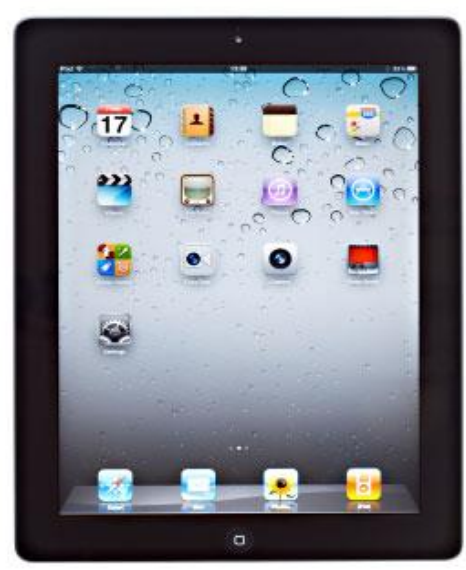

Abb. 27: Darstellung eines iPads ${ }^{\circledR}$

Vorausgegangen war zunächst die PDA-Reihe (persönlicher digitaler Assistent) Newton in den 1990er Jahren und 2007 schließlich das Smartphone-Modell iPhone $\AA$, das als eine Folgeentwicklung dieser Mini-Computer-Technologie zu betrachten ist.

Das iPad® der ersten Generation wird mit einem Apple-A4-Prozessor mit einer Taktfrequenz von $1 \mathrm{GHz}$ betrieben und hat einen Arbeitsspeicher von $256 \mathrm{MB}$. Für den Flash-Speicher stehen drei Varianten zur Auswahl, mit 16 GB, 32 GB oder 64 GB Kapazität. Das iPad verfügt über eine Wi-Fi-Schnittstelle für den Internetzugriff innerhalb von WLAN-Netzwerken und ist zudem geeignet für die mobile Internetnutzung über den 2,5G- und den 3G-Funkstandard. Die Bedienung erfolgt über das kapazitive 9,7-Zoll-Multi-Touch Display, eine Kamera ist in der ersten Generation nicht integriert. International konnte das $\mathrm{IPad} \circledast$ seit Sommer 2010 erworben werden.

Das iPad2® kam im Jahr 2011 auf den Markt und wartete mit diversen Neuerungen und Verbesserungen auf. Integriert ist hier ein Dual-Core-A5Prozessor mit 512 MB Arbeitsspeicher. Außerdem verfügt das Gerät über zwei digitale Kameras sowie ein 3-Achsen-Gyroskop, das Rotation erfassen kann und den Bildschirm entsprechend ausrichtet.

2012 erschien das New iPad - oder auch iPad3® - als erneute Weiterentwicklung mit Dual-Core-A5X-Prozessor, 1024 MB Arbeitsspeicher, 
Quad-Core-Grafikchip für noch schärfere HD-Bildschirmauflösung sowie mit leistungsstärkeren Kameras (inklusive Gesichtserkennung) und einer Variante des bereits im iPhone $4 S \AA$ integrierten Sprachsteuerungsprogramms Siri für das Diktieren von Texten (Tablet-PCs.eu, 2015a). Inzwischen sind 2012 das iPad 4® sowie das iPad mini® entwickelt worden. 2013 erschienen auf dem Markt das iPadAir $\circledast$ sowie das iPad mini® mit Retina Display und 2014 das iPadAir2 $\circledast$ und das iPadmini3®. Das iPad Air® verfügt ein 9,7"-Retina-Display, das neue iPad Air $2 \AA$ bietet dazu ein Touch-ID, den schnellen A8X-Chip und neue Kameras. Das Design ist stets dünn und leicht. Das iPadAir ${ }^{\circledR}$ und iPadAir $2 \AA$ sowie die leichteren neueren Modelle des iPad mini® bieten zusätzlich eine iSight-Kamera, womit eine Gesichtserkennung und sowie HDR-Fotos (High Dynamic Range Image (HDRI), „Bild mit hohem Dynamikumfang“) und Panorama-Bilder gewährleistet werden können (iPad, 2015a, 2015b).

Alle iPad-Generationen basieren auf dem Apple eigenen, auch für das iPhone verwendeten Betriebssystem iOS, welches seit dem 29. Juli 2007 von Apple Inc. verwendet wird. Es ist multitaskingfähig, sodass verschiedene Anwendungen gleichzeitig ausführbar sind und kann durch eine Vielzahl von Applikationen aus dem Apple Store ergänzt werden (Diffen, 2015; Tablet-PCs.eu, 2015a).

\subsubsection{Farbdisplay von Tablet-PCs - Aufbau und Funktionsweise}

\section{Farbdisplay}

Die verschiedenen Tablet-PC-Modelle zeigen Unterschiede hinsichtlich der Farbqualität, da diese auch über verschiedene Displays verfügen.

Das iPad1® und iPad $2 \AA$ verfügen über ein 9,7“-Multi-Touch-Widescreen-Display mit LED (Light-emitting Diode, dt. licht-emittierende Diode, auch LumineszenzDiode), mit Hintergrundbeleuchtung, Hochglanzanzeige und IPS (In-PlaneSwitching)-Technologie sowie mit einer Auflösung von 1024x768 Pixeln bei 132 ppi. Das iPad $3 \AA$ verfügt über ein Retina-Display mit 9,7“-Multi-TouchHochglanz-Widescreendisplay mit LED-Hintergrundbeleuchtung und IPSTechnologie sowie mit einer höheren Auflösung von 2048x1536 Pixeln bei 264 
ppi (iPad, 2015b). Die weiterentwickelten iPads, iPad mini 2 und $3 \AA$, und iPad Air ${ }^{\circledR}$ und iPad Air $2 \AA$, verfügen ebenfalls über ein Retina-Display und weisen höhere Auflösungen auf. Als Retina-Display bezeichnet das US-amerikanische Unternehmen Apple Bildschirme mit einer Pixeldichte, die so hoch ist, dass das menschliche Auge aus einem typischen Betrachtungsabstand nicht in der Lage sein soll, einzelne Pixel zu erkennen. Alle bisher von Apple verbauten RetinaDisplays sind Flüssigkristallanzeigen (LCDs) mit In-Plane Switching (IPS). Die folgende Tabelle (Tab. 2) gibt eine Übersicht über ihre Punktdichten (in Pixel pro Zoll) und Bildschirmauflösungen (Wikipedia, 2015c):

Tab. 2: Darstellung der Punktdichten (in Pixel pro Zoll) und Bildschirmauflösungen von verschiedenen iPad®-Generationen (Wikipedia, 2015c)

\begin{tabular}{|l|l|l|}
\hline Produkt & $\begin{array}{l}\text { Punktdichte } \\
\text { in ppi }\end{array}$ & $\begin{array}{l}\text { Bildschirmauflösung } \\
\text { in Pixel }\end{array}$ \\
\hline $\mathrm{iPad}$ mini® (2./3.Generation) & 326 & $2048 \times 1536$ \\
\hline $\begin{array}{l}\mathrm{iPad}(\text { (3./4. Generation), iPad Air® } \\
\text { (1./2. Generation) }\end{array}$ & 264 & $2018 \times 1536$ \\
\hline
\end{tabular}

\section{$\underline{\text { Touchscreen }}$}

Ein Touchscreen ist ein kombiniertes Ein- und Ausgabegerät, bei dem durch Berührung von Teilen eines Bildes der Programmablauf eines technischen Gerätes direkt gesteuert werden kann. Die technische Umsetzung der Befehlseingabe ist für den Nutzer gleichsam unsichtbar und erzeugt so den Eindruck einer unmittelbaren Steuerung des Computers oder Tablet-PCs per Fingerzeig. Statt einen Mauszeiger mit der Maus oder Ähnlichem zu steuern, kann der Finger oder ein Zeigestift verwendet werden. Die Analogie zum Mausklick ist ein kurzes Tippen. Durch Ziehen des Fingers oder Stiftes über den Touchscreen kann eine „Ziehen und Fallenlassen“-Operation ausgeführt werden. Die iPads $\circledast$ können mehrere gleichzeitige Berührungen zu Befehlen verarbeiten (Multi-Touch), um zum Beispiel angezeigte Elemente zu drehen oder zu skalieren. Der Begriff „Multi-Touch“ wird meistens auch im Zusammenhang 
benutzt mit der Fähigkeit des Systems, Gesten zu erkennen, z. B. „Wischen“, um eine Seite umzublättern.

Andere Systeme erlauben, z. B. durch die berührungslose Erkennung eines darüber schwebenden Fingers, die volle Emulation eines Mauszeigers mit einem vom Tippen verschiedenen reinen Zeigemodus.

Es gibt mehrere Funktionsprinzipien zur Umsetzung der Berührungsempfindlichkeit: resistive Systeme, oberflächen-kapazitive Systeme, projiziert-kapazitive Systeme, induktive Systeme, SAW (Surface Acoustic Wave), d. h. "(schall)wellen-gesteuerte Systeme“, optische Systeme (in der Regel Infrarotlicht-Gitter vor dem Monitor), Dispersive-Signal-Technology-Systeme.

Der projiziert-kapazitive Touchscreen bei iPads verwendete Variante. Dieser projiziert-kapazitive Touchscreen (meist „PCT“ = „Projected Capacitive Touch“ oder "PCAP“ abgekürzt) nutzt zwei Ebenen mit einem leitfähigen Muster (meistens Streifen oder Rauten). Die Ebenen sind voneinander isoliert angebracht. Eine Ebene dient als Sensor, die andere übernimmt die Aufgabe des Treibers. Befindet sich ein Finger am Kreuzungspunkt zweier Streifen, so ändert sich die Kapazität des Kondensators, und es kommt ein stärkeres Signal am Empfängerstreifen an. Der wesentliche Vorteil dieses Systems ist, dass der Sensor auf der Rückseite des Deckglases angebracht werden kann. So erfolgt die Bedienung auf der praktisch verschleißfreien Glasoberfläche. Ferner ist die Erkennung von Gesten und mehreren Berührungen (also Multi-Touch) möglich. Kapazitive Touchscreens können nur mit dem bloßen Finger, leitfähigen Eingabestiften oder speziell angefertigten Hilfsmitteln, nicht aber mit einem herkömmlichen Eingabestift oder dicken Handschuhen bedient werden (Wikipedia, 2015f) (Abb. 28). 


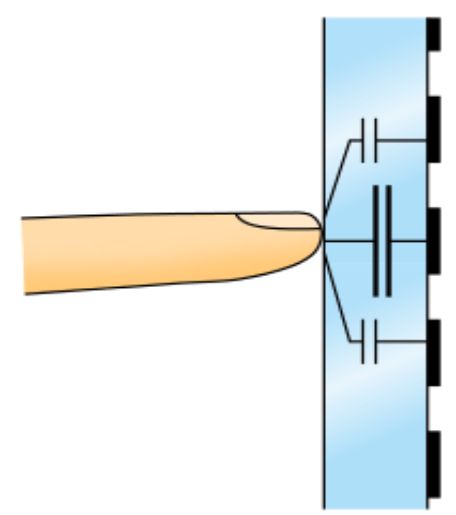

Abb. 28: Projiziert-kapazitiver Touchscreen

\section{$\underline{\text { Farbmanagement }}$}

Hinsichtlich des Farbmanagements bietet das iPad® grundsätzlich keine Kalibrierungsmöglichkeiten und, abgesehen von der Helligkeit, auch keine hardwareseitige Möglichkeit der Bildschirmeinstellung. Daher kann eine verlässliche Farbverbindlichkeit kaum möglich sein. Ein Vergleich zwischen mehreren Geräten lässt erhebliche Unterschiede in der Farbtemperatur der Bildschirme erkennen. Ein Layout oder Bild auf unterschiedlichen Geräten kann daher im einen Fall bläulich, im anderen gelblich oder zu rot erscheinen.

Das Betriebssystem iOS verfügt über kein zentrales CMS (colormanagementsystem), wie dies bei den Mac-Computern in Form von ColorSync beispielsweise der Fall ist.

Außerdem zeigt sich bei der Vermessung des iPads $\AA$, dass dessen Farbraum kleiner als der sRGB-Farbraum ist. Demzufolge können viele Farben auf dem Gerät nicht dargestellt werden, und gerade die Grauachse begrenzt die Möglichkeiten von Zeichnung in hellen und dunklen Bereichen (Benkert, 2012) (Abb. 29). 


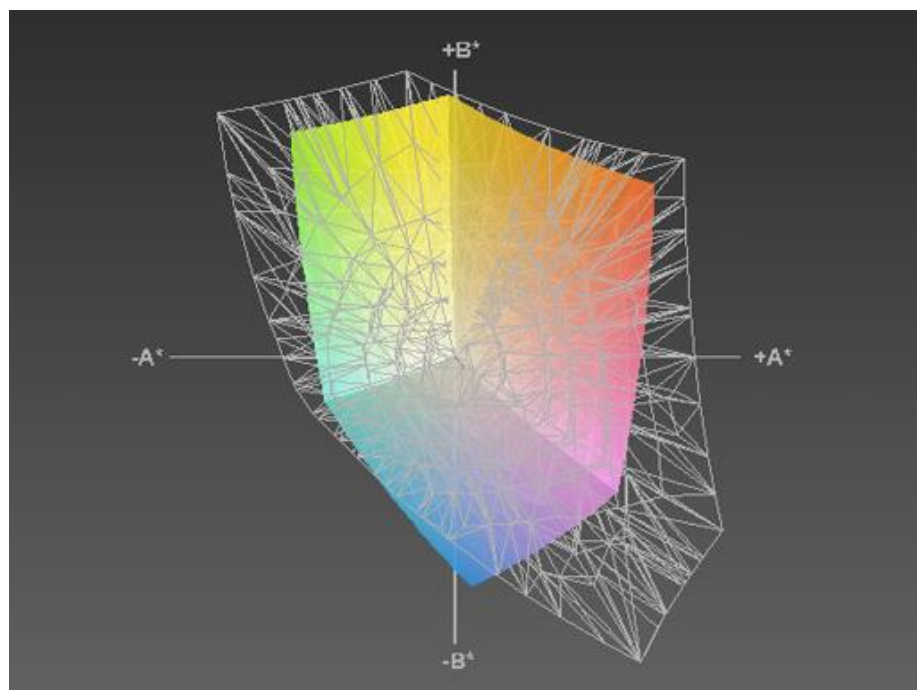

Abb. 29: iPad®-Farbraum vs. sRGB-Farbraum (Grid)

Im September 2008 wurden das Betriebssystem und die Software-Plattform Android (Fa. Google) für mobile Geräte wie Smartphones, Mobiltelefone, Mediaplayer, Netbooks und Tablet-Computer entwickelt (Wikipedia, 2015a). Viele Smartphones und Tablet-PCs wie Kindle Fire (modifiziertes Android) und die Geräte der Firmen LG, HTC, Samsung, Sony, Motorola, Nexus und vieler anderer verfügen über die Android-Software (Diffen, 2015). Es sind verschiedene Displays mit unterschiedlichem Umfang der Darstellung des Farbraumes verfügbar. Den darstellbaren Farbraum verschiedener Tablet-PCs zeigt Abb. 30.

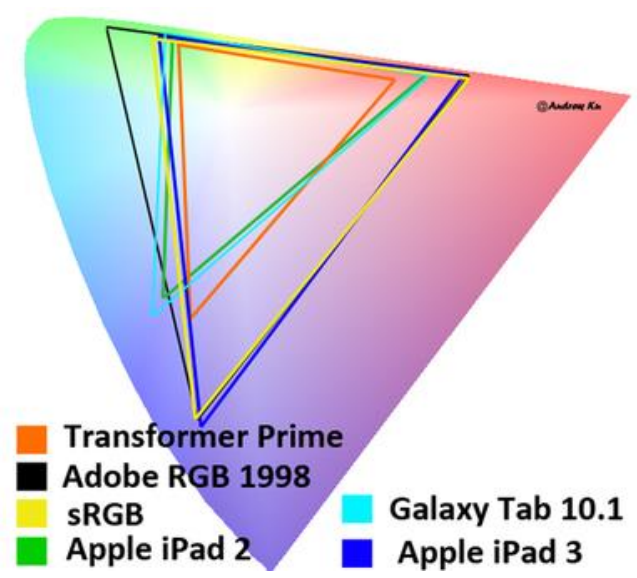

Abb. 30: Farbraumdarstellung von verschiedenen Tablet-PCs (M.Wellendorf, 2012) 


\section{Methodik}

\subsection{Probandenrekrutierung}

Die Rekrutierung von Probanden erfolgte durch gezielte Informationen im Internet (E-Mail-Verteiler der Helios Klinik Berlin Buch und das lokale Kleinanzeigenportal: das Schwarze Brett) sowie in den lokalen Printmedien („Bucher Bote“). Die Untersuchungen erfolgten unter standardisierten Arbeitsbedingungen im Zeitraum von Mai 2012 bis Dezember 2013.

Es wurden insgesamt 77 freiwillige Probanden untersucht. 62 davon waren normalsichtig und 15 hatten eine Farbsehstörung. Zudem erfolgte bei 10 Probanden ein Vergleich zwischen iPad $1 \circledR$ und iPad $2 \circledast$.

Es wurden keine Patienten eingeschlossen und die Untersuchungen waren nicht mit Belastungen oder Risiken für die Probanden verbunden. Die Probanden wurden umfassend über Ziele und Verfahren der Untersuchung aufgeklärt.

\subsection{Verwendete Farbtafeln und verwendetes Anomaloskop}

Es sind 19 Pigmentfarbtafeln nach Velhagen, Broschmann und Kuchenbecker und die gleichen Tafeln in digitaler Form mittels Tablet-PC verglichen worden. Es wurden die Farbtafeln nach Velhagen, Broschmann und Kuchenbecker „Tafeln zur Prüfung des Farbensinnes" in der 34. Auflage (2011) verwendet (s. Anhang 1) (Abb. 31, Tab. 3).

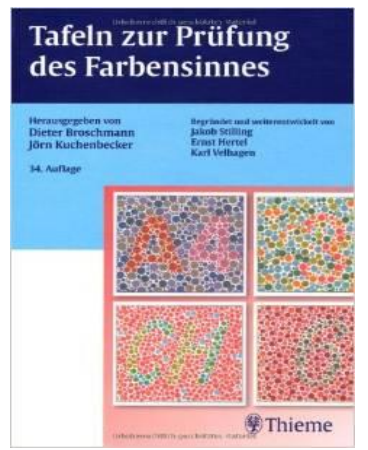

Abb. 31: Tafeln zur Prüfung des Farbsehens, 34. Auflage (2011) 
Tab. 3: Verwendete Farbtafeln

\begin{tabular}{|c|c|c|c|}
\hline Tafel-Nr. & Antwort Farbnormaler & Antwort Farbfehlsichtiger & Beschreibung \\
\hline 1 & $2 \mathrm{~L}$ & $2 \mathrm{~L}$ & Demonstrationstafel \\
\hline 2 & 182 & $\begin{array}{l}\text { Rot-Grün-Störung: } 8 \\
\text { Blau-Gelb-Störung: } 12\end{array}$ & diagnostische Tafel \\
\hline 3 & 69 & Rot-Grün-Störung: 60, 66, 00 & $\begin{array}{l}\text { Tafel mit alternativem } \\
\text { Sehzeichen }\end{array}$ \\
\hline 4 & $\mathrm{H} 5$ & $\begin{array}{l}\text { Rot-Grün-Störung: keine oder } \\
\text { falsche Antwort }\end{array}$ & $\begin{array}{l}\text { Tafel mit verschwin- } \\
\text { denden Sehzeichen }\end{array}$ \\
\hline 5 & 3 & $\begin{array}{l}\text { Rot-Grün-Störung: keine oder } \\
\text { falsche Antwort }\end{array}$ & $\begin{array}{l}\text { Tafel mit verschwin- } \\
\text { denden Sehzeichen }\end{array}$ \\
\hline 6 & $6 R$ & $\begin{array}{l}\text { Rot-Grün-Störung: keine oder } \\
\text { falsche Antwort }\end{array}$ & $\begin{array}{l}\text { Tafel mit verschwin- } \\
\text { denden Sehzeichen }\end{array}$ \\
\hline 7 & 2 & $\begin{array}{l}\text { Rot-Grün-Störung: keine oder } \\
\text { falsche Antwort }\end{array}$ & $\begin{array}{l}\text { Tafel mit verschwin- } \\
\text { denden Sehzeichen }\end{array}$ \\
\hline 8 & F4 & $\begin{array}{l}\text { Rot-Grün-Störung: keine oder } \\
\text { falsche Antwort }\end{array}$ & $\begin{array}{l}\text { Tafel mit verschwin- } \\
\text { denden Sehzeichen }\end{array}$ \\
\hline 9 & 6 & $\begin{array}{l}\text { Rot-Grün-Störung: keine oder } \\
\text { falsche Antwort }\end{array}$ & $\begin{array}{l}\text { Tafel mit verschwin- } \\
\text { denden Sehzeichen }\end{array}$ \\
\hline 10 & 51 & $\begin{array}{l}\text { Rot-Grün-Störung: keine oder } \\
\text { falsche Antwort }\end{array}$ & $\begin{array}{l}\text { Tafel mit verschwin- } \\
\text { denden Sehzeichen }\end{array}$ \\
\hline 11 & 3 & Rot-Grün-Störung: 8 & $\begin{array}{l}\text { Tafel mit alternativem } \\
\text { Sehzeichen }\end{array}$ \\
\hline 12 & $\mathrm{CH}$ & $\begin{array}{l}\text { Rot-Grün-Störung: } \\
\text { 31oder } 37\end{array}$ & $\begin{array}{l}\text { Tafel mit alternativem } \\
\text { Sehzeichen }\end{array}$ \\
\hline 13 & 42 & $\begin{array}{l}\text { Rot-Grün-Störung: keine oder } \\
\text { falsche Antwort }\end{array}$ & $\begin{array}{l}\text { Tafel mit verschwin- } \\
\text { denden Sehzeichen }\end{array}$ \\
\hline 14 & 49 & $\begin{array}{l}\text { Blau-Gelb-Störung: keine } \\
\text { oder falsche Antwort }\end{array}$ & $\begin{array}{l}\text { Tafel mit verschwin- } \\
\text { denden Sehzeichen }\end{array}$ \\
\hline 15 & $5 \mathrm{E}$ & $\begin{array}{l}\text { Blau-Gelb-Störung: keine } \\
\text { oder falsche Antwort }\end{array}$ & $\begin{array}{l}\text { Tafel mit verschwin- } \\
\text { denden Sehzeichen }\end{array}$ \\
\hline 16 & 65 & $\begin{array}{l}\text { Rot-Grün-Störung: keine oder } \\
\text { falsche Antwort }\end{array}$ & $\begin{array}{l}\text { Tafel mit verschwin- } \\
\text { denden Sehzeichen }\end{array}$ \\
\hline 17 & RG & $\begin{array}{l}\text { Rot-Grün-Störung: keine oder } \\
\text { falsche Antwort }\end{array}$ & $\begin{array}{l}\text { Tafel mit verschwin- } \\
\text { denden Sehzeichen }\end{array}$ \\
\hline 18 & A4 & $\begin{array}{l}\text { Rot-Grün-Störung: keine oder } \\
\text { falsche Antwort }\end{array}$ & $\begin{array}{l}\text { Tafel mit verschwin- } \\
\text { denden Sehzeichen }\end{array}$ \\
\hline 19 & B5 & Rot-Grün-Störung: B6 & $\begin{array}{l}\text { Tafel mit alternativem } \\
\text { Sehzeichen }\end{array}$ \\
\hline
\end{tabular}


Als Anomaloskop wurde das Heidelberger Multi-Color-Anomaloskop der Fa. Oculus verwendet (Abb. 32).

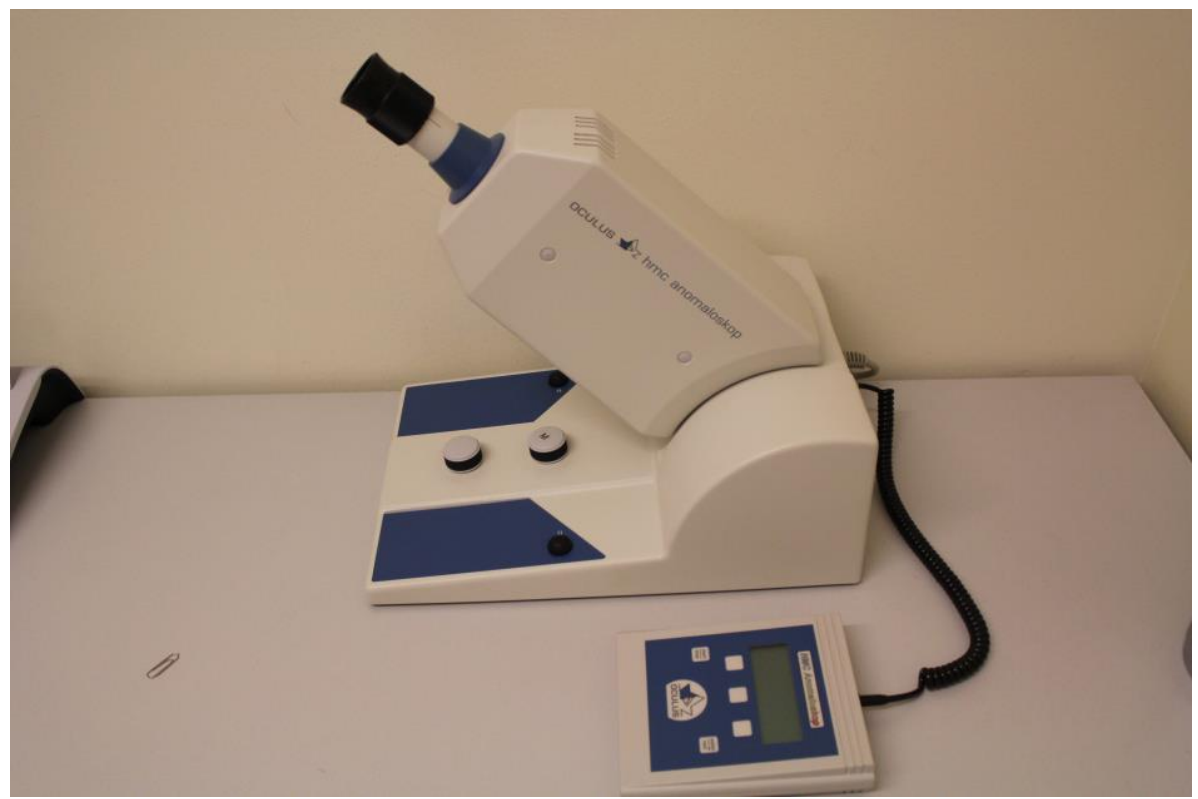

Abb. 32: Verwendetes HMC-Anomaloskop

\subsection{Durchführung der Tests}

Es wurden die Ergebnisse der Farbsehtestung mittels Pigmentfarbtafeln und Tablet-PC (iPad2 $\AA$ ) in einer prospektiven, randomisierten Studie miteinander verglichen. Dabei wurde immer nur das rechte Auge geprüft. Zunächst erfolgte sine bzw. cum correctione eine Visusprüfung für die Nähe. An geraden Tagen erfolgte zunächst die Untersuchung mit dem Buch, danach die mit dem TabletPC, und an ungeraden Tagen wurde die Reihenfolge umgekehrt. Die Untersuchung erfolgte unter standardisierten Bedingungen. Die Untersuchung mit dem Buch wurde unter Verwendung einer Tageslichtlampe mit standardisierter Beleuchtung von $6500 \mathrm{~K}$ und einer Beleuchtungsstärke 800 lux durchgeführt. Die Untersuchung mit dem Tablet-PC wurde bei einer Einstellung des Displays auf maximale Helligkeit durchgeführt. Der Untersuchungsabstand betrug $70 \mathrm{~cm}$. Anschließend erfolgte die Untersuchung mit dem HMCAnomaloskop der Firma Oculus. Es wurde die Rayleigh-Gleichung verwendet. Dabei wurde der Anomalquotient bestimmt (Abb. 33, Abb. 34). 


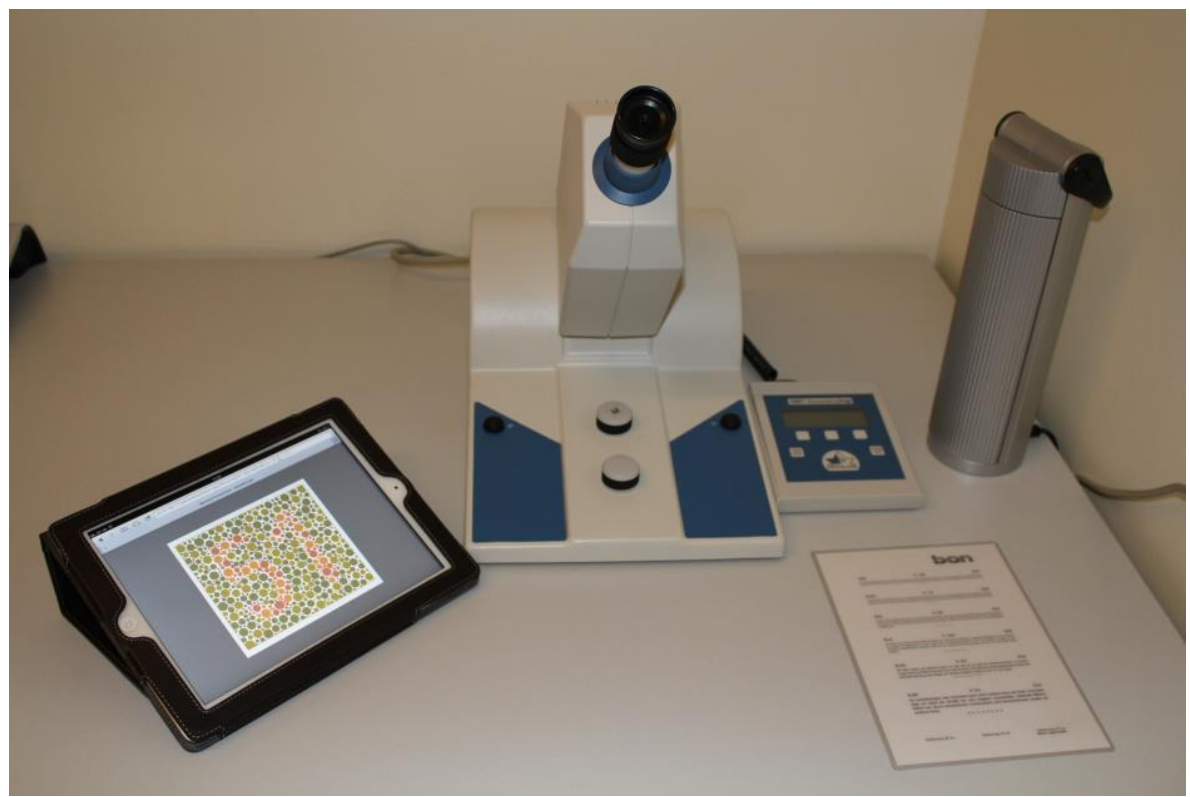

Abb. 33: Untersuchungsplatz

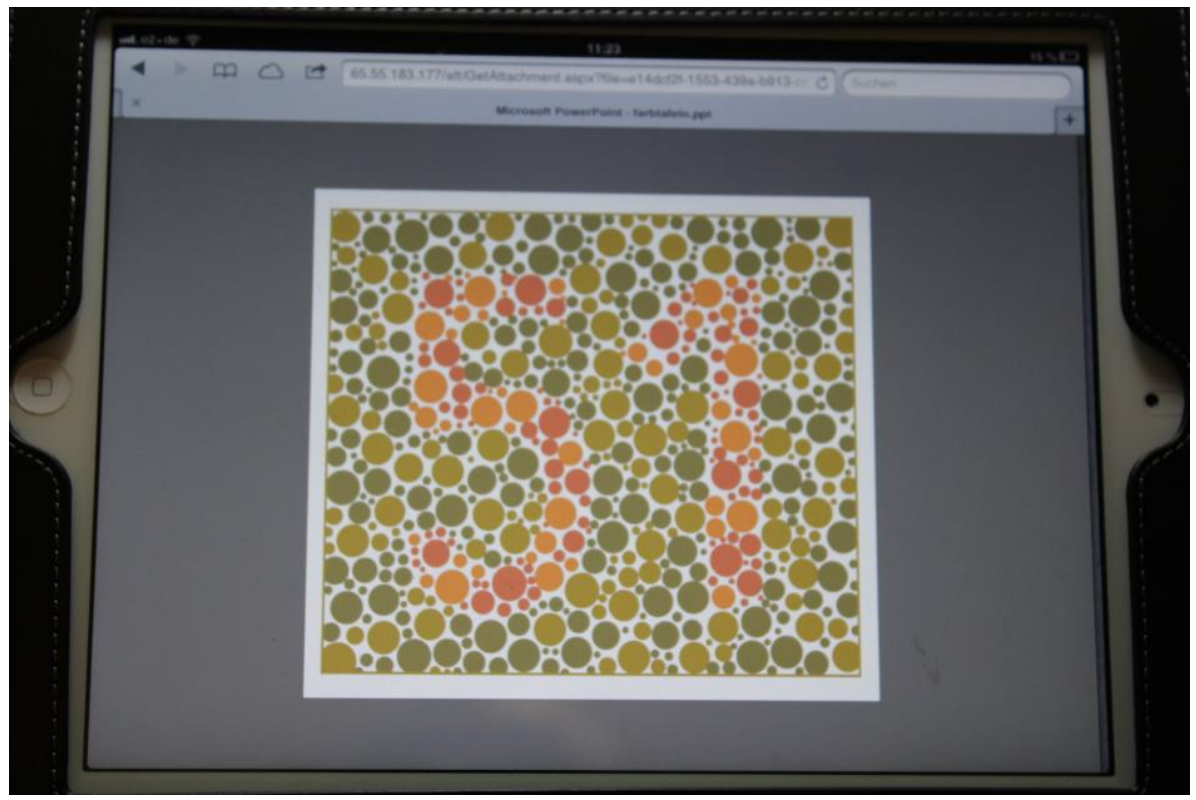

Abb. 34: Verwendetes $\mathrm{iPad}{ }^{\circledR}$ mit Darstellung einer Farbtafel

Wenn es bei der Untersuchung mit dem Anomaloskop zu dem Ergebnis „Diagnose nicht möglich“ kam, erfolgte eine Untersuchung mit den pseudoisochromatischen Ishihara-Farbtafeln Nummer 15 und 17 der Auflage von 1979. Damit konnte eine Deuteranomalie bzw. eine Deuteranopie von einer 
Protanomalie bzw. Protanopie differenziert werden. Die Tafel 15 wurde bereits in der Abbildung 22 dargestellt, hier wird die Tafel 17 gezeigt (Abb. 35).

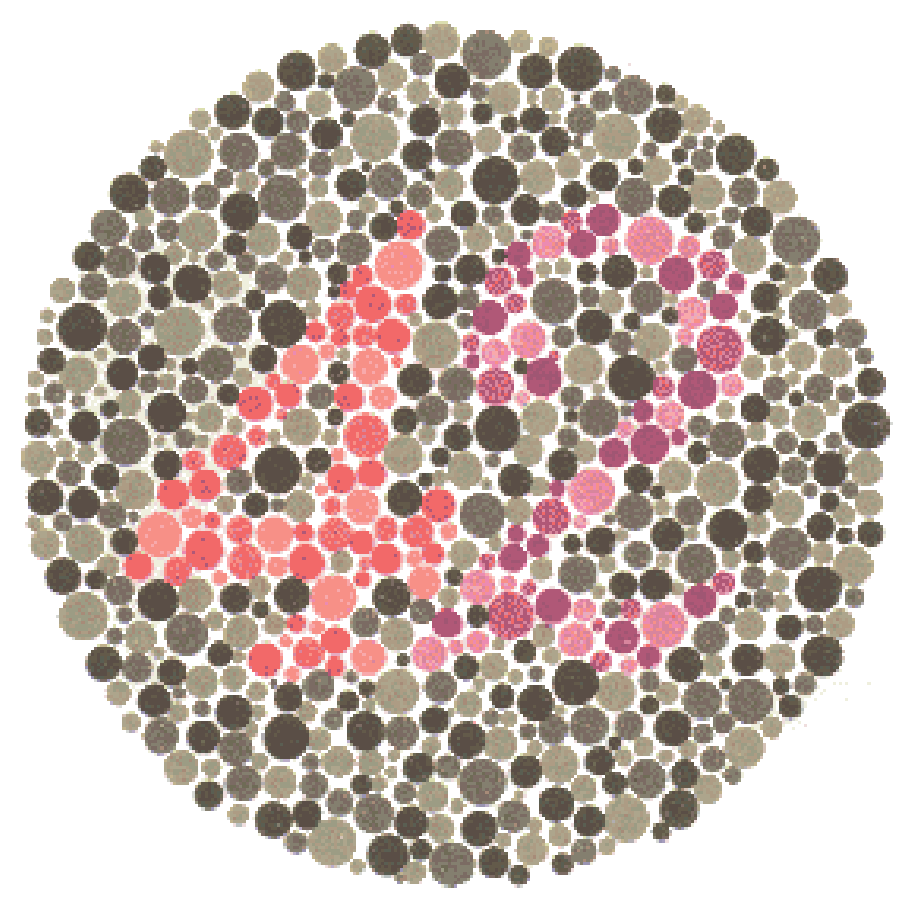

Abb. 35: Ishihara-Farbtafel 17 (Antwort 4: Deuteranomalie, Antwort 2: Protanomalie)

Außerdem wurden zehn Probanden (ein Proband mit Farbsehstörung und neun Probanden ohne Farbsehstörung) mit dem iPad1® untersucht.

\subsection{Statistische Auswertung}

Die statistische Auswertung erfolgte mithilfe der Software „SPSS“ (Version 13, SPSS Inc., Illinois, USA).

Es wurde für jede Tafel geprüft, ob zwischen den Angaben „Buch“ vs. „iPad2®“ bzw. „iPad1@“ Übereinstimmung bestand. Auch die Angabe „ng“ (nicht gelesen) in beiden Fällen wurde als Übereinstimmung gewertet.

Die Konfidenzintervalle wurden nach der Wilson-Score-Methode berechnet. 
Zunächst wurde das iPad2® mit dem Buch bei allen Probanden verglichen.

Dann wurde das iPad1 $\AA$ mit dem iPad $2 \AA$ und das iPad $1 \AA$ mit dem Buch verglichen.

Anschließend erfolgte ein Vergleich zwischen den drei Gruppen: Buch vs. iPad1 $\AA$, Buch vs. iPad $2 \AA$, iPad1 $\AA$ vs. iPad $2 \AA$. Die Übereinstimmung zwischen den drei Gruppen wurde nach Landis und Koch eingeteilt (Landis et al., 1977). 


\section{Ergebnisse der statistischen Auswertung}

\subsection{Alters- und Geschlechtsverteilung}

Das Durchschnittsalter aller Probanden betrug 42,81 \pm 16,95 Jahre (Abb. 36). Es wurden insgesamt 77 Probanden, davon 35 Männer und 42 Frauen untersucht. Von den Männern hatten 15 eine Farbsehstörung und 20 keine Farbsehstörung. Bei den Frauen wurde keine Farbsehstörung festgestellt. Die Signifikanz wurde mittels des Zweistichproben-t-Tests (Welch-Test oder t-Test nach Satterthwaite) berechnet. Der $\mathrm{p}$-Wert für die Altersverteilung zwischen Farbnormalen und Farbfehlsichtigen betrug $0,14(p>0,1)$, sodass kein signifikanter Unterschied bestand.

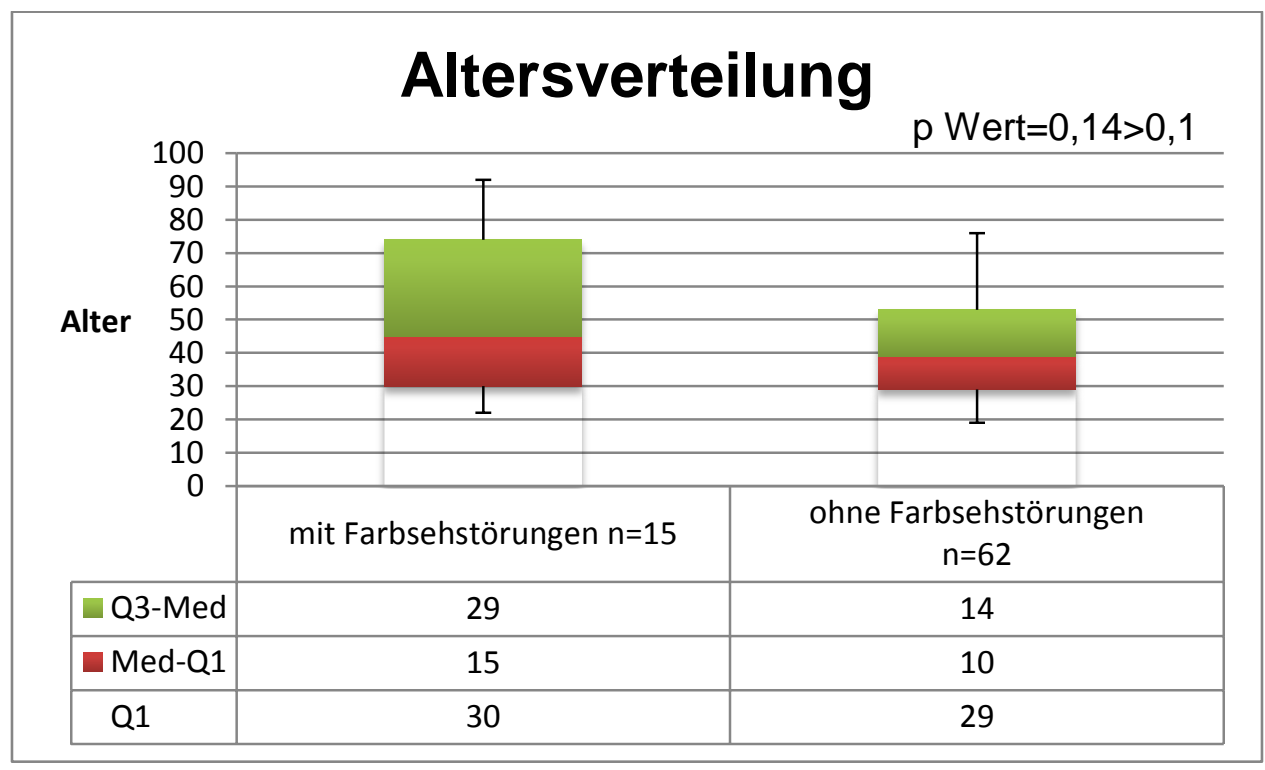

Abb. 36: Darstellung der Altersverteilung der Probanden mit und ohne Farbsehstörung (Minimum=22, Q1=30, Median=45, Q3=74, Maximum=92 bei den Farbfehlsichtigen, Minimum=19, Q1=29, Median=39, Q3=53, Maximum=76 bei den Farbnormalen)

\subsection{Visusergebnisse}

Der mittlere Nahvisus betrug insgesamt 0,99 $\pm 0,15$ für alle Probanden (Abb. 37). Der p-Wert für den Nahvisus der Farbnormalen und der Farbfehlsichtigen betrug $0,41$ ( $p>0,1)$, sodass kein signifikanter Unterschied bestand. 


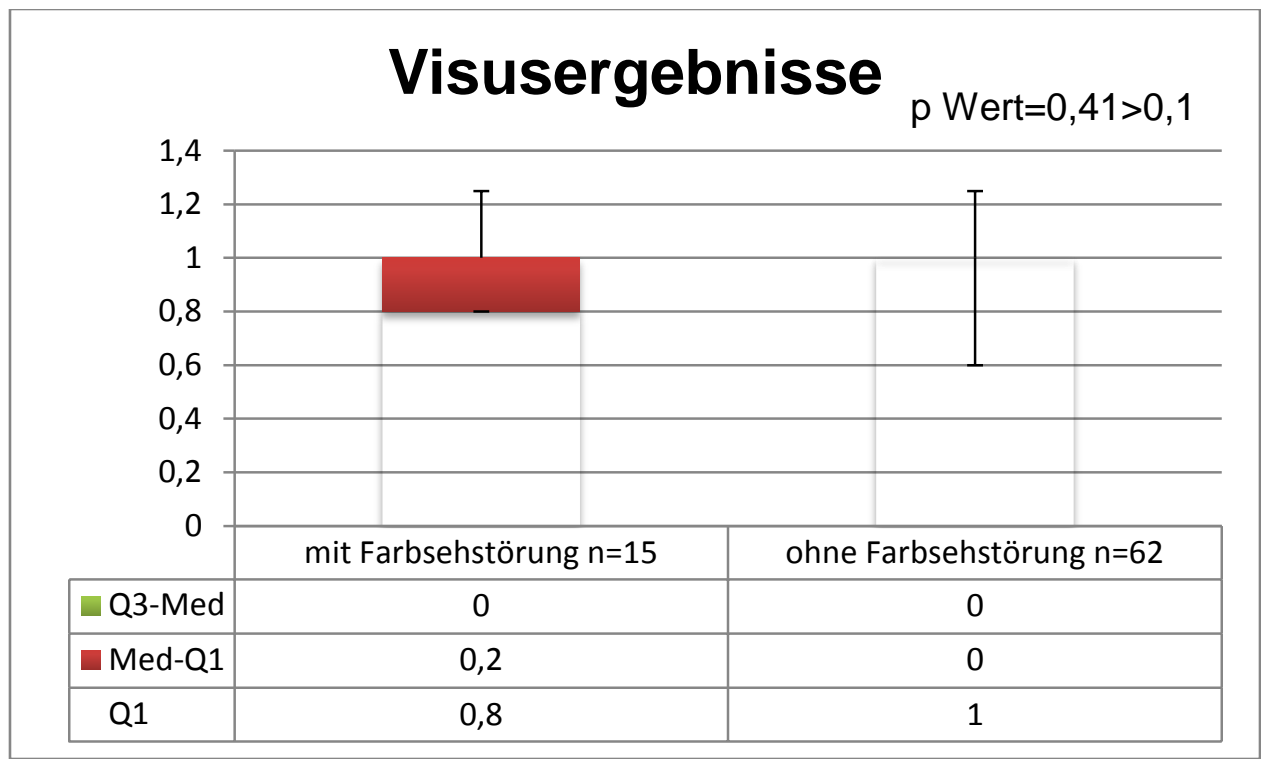

Abb. 37: Darstellung der Visusverteilung der Probanden mit und ohne Farbsehstörung (Minimum=0,8, Q1=0,8, Median=1, Q3=1, Maximum=1,25 bei den Farbfehlsichtigen, Minimum=0,6, $Q 1=1$, Median=1, $Q 3=1$, Maximum=1,25 bei den Farbnormalen)

\subsection{Verteilung der Farbsehstörungen}

Von den 77 Probanden hatten 15 eine angeborene Farbsehstörung. Diese Probanden waren alle männlich. 8 Probanden hatten eine Deuteranomalie, 5 eine Deuteranopie und 2 eine Protanomalie.

\subsection{Verteilung der Fehler}

Es folgt die Darstellung der Anzahl der Fehler bei den Probanden mit und ohne Farbsehstörung bei der Untersuchung des Farbsehens mittels Buch (Abb. 38) und iPad2® (Abb. 39). 


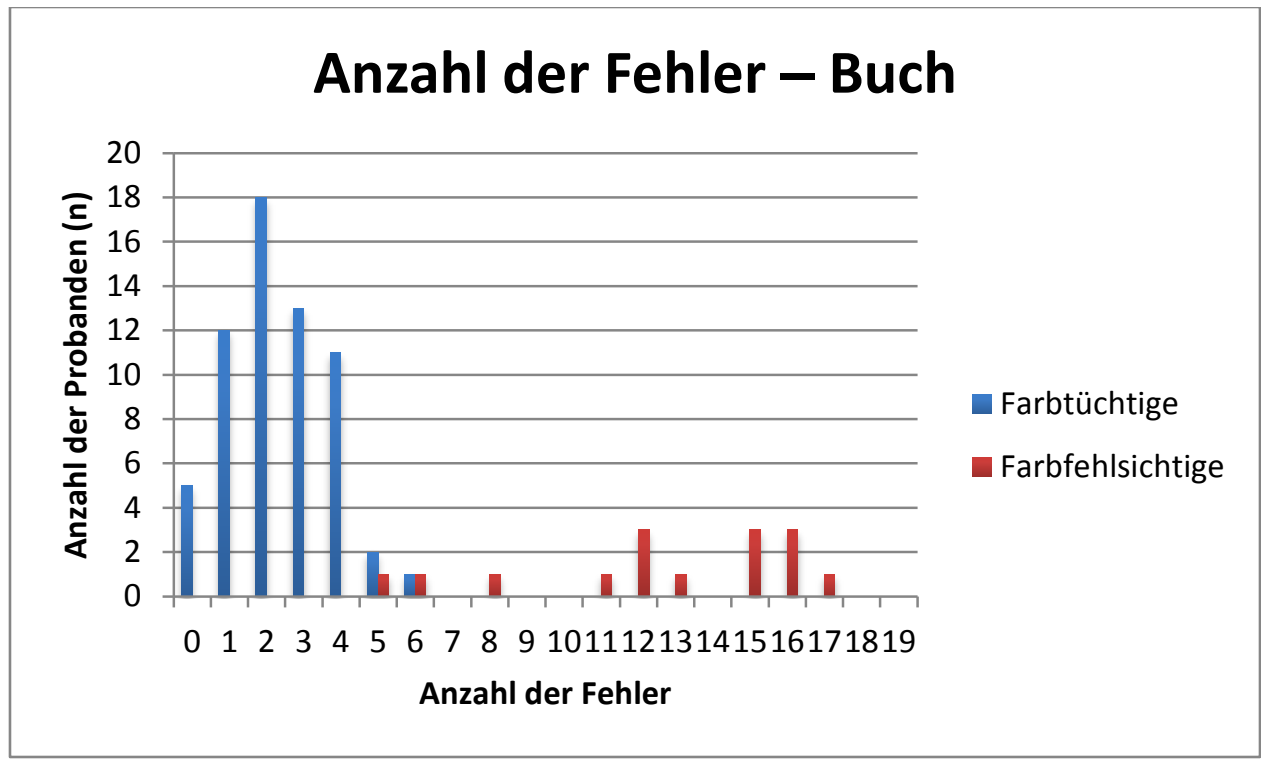

Abb. 38: Darstellung der Anzahl der Fehler pro Tafel bei der Untersuchung des Farbsehens mittels Buch bei den Probanden mit Farbsehstörung (rot) und ohne Farbsehstörung (blau), $n=$ Anzahl der Probanden

Es zeigt sich, dass ein Proband der Gruppe der Farbtüchtigen 6 Fehler aufwies und ein Proband der Gruppe der Farbfehlsichtigen 5 Fehler hatte.

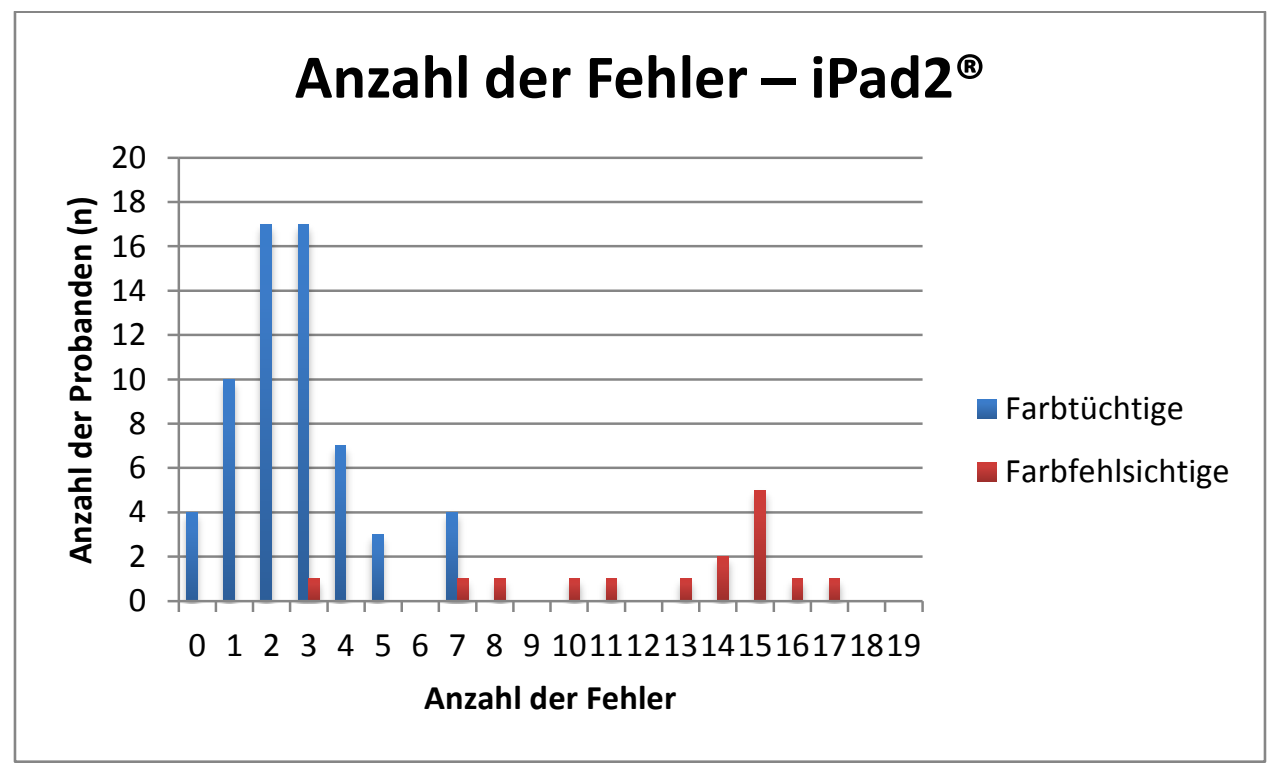

Abb. 39: Darstellung der Anzahl der Fehler pro Tafel bei der Untersuchung des Farbsehens mittels iPad2 ${ }^{\circledR}$ bei den Probanden mit Farbsehstörung (rot) und ohne Farbsehstörung (blau), $\mathrm{n}=$ Anzahl der Probanden

Es zeigt sich hier, dass ein Proband der Gruppe der Farbtüchtigen 7 Fehler hatte, während ein Proband der Gruppe der Farbfehlsichtigen nur 3 Fehler 
aufwies. Die Trennschärfe des $\mathrm{iPad} 2 \AA$ ist damit etwas geringer als die des Buches.

\subsection{Vergleich des Farbsehens anhand verschiedener Medien}

\subsubsection{Vergleich des Farbsehens mittels Buch und iPad2® bei allen Probanden}

Die Übereinstimmung beim Vergleich der 19 Velhagen/Broschmann/ Kuchenbecker-Farbtafeln zwischen Buch und iPad2® bei allen Nutzern betrug $88 \%$ (Tab. 4). Das 95\%-Konfidenzintervall reicht von 81,6 bis $89,6 \%$.

Tab. 4: Vergleich des Farbsehens zwischen Buch und $\mathrm{iPad} 2 \AA$ bei allen Probanden

\begin{tabular}{|c|c|c|c|c|c|}
\hline & & Häufigkeit & Prozent & $\begin{array}{l}\text { Gültige } \\
\text { Prozente }\end{array}$ & $\begin{array}{l}\text { Kumulierte } \\
\text { Prozente }\end{array}$ \\
\hline \multirow{3}{*}{ Gültig } & keine Übereinstimmung & 167 & 12,0 & 12,0 & 12,0 \\
\hline & $\begin{array}{l}\text { Übereinstimmung } \\
\text { (Buch=iPad2®) }\end{array}$ & 1219 & 88,0 & 88,0 & 100,0 \\
\hline & Gesamt & 1386 & 100,0 & 100,0 & \\
\hline
\end{tabular}

Die höchste Übereinstimmung besteht mit 100\% bei der Tafel 14 (Zeichen: 49). Die geringste Übereinstimmung besteht mit $55,8 \%$ bei der Tafel 4 (Zeichen: H5) (Tab. 5). 
Tab. 5: Auswertung der Ergebnisse pro Tafel zwischen Buch und $\mathrm{iPad} 2 \AA$ bei allen Nutzern

\begin{tabular}{|c|c|c|c|c|c|c|}
\hline & $\begin{array}{l}\text { Keine } \\
\text { Übereinstimmung }\end{array}$ & $(\%)$ & Übereinstimmung & $(\%)$ & Gesamt & $\begin{array}{l}95 \%- \\
\text { Konfidenzintervall }\end{array}$ \\
\hline 2 & 12 & $15,6 \%$ & 65 & $84,4 \%$ & 77 & {$[74,7 \%, 90,9 \%]$} \\
\hline 3 & 2 & $2,6 \%$ & 75 & $97,4 \%$ & 77 & {$[91,0 \%, 99,3 \%]$} \\
\hline 4 & 34 & $44,2 \%$ & 43 & $55,8 \%$ & 77 & {$[44,7 \%, 66,4 \%]$} \\
\hline 5 & 1 & $1,3 \%$ & 76 & $98,7 \%$ & 77 & {$[93,0 \%, 99,8 \%]$} \\
\hline 6 & 8 & $10,4 \%$ & 69 & $89,6 \%$ & 77 & {$[80,8 \%, 94,6 \%]$} \\
\hline 7 & 3 & $3,9 \%$ & 74 & $96,1 \%$ & 77 & {$[89,2 \%, 98,7 \%]$} \\
\hline 8 & 3 & $3,9 \%$ & 74 & $96,1 \%$ & 77 & {$[89,2 \%, 98,7 \%]$} \\
\hline 9 & 15 & $19,5 \%$ & 62 & $80,5 \%$ & 77 & {$[70,3 \%, 87,8 \%]$} \\
\hline 10 & 2 & $2,6 \%$ & 75 & $97,4 \%$ & 77 & {$[91,0 \%, 99,3 \%]$} \\
\hline Tafel & & & & & & \\
\hline 11 & 5 & $6,5 \%$ & 72 & $93,5 \%$ & 77 & {$[85,7 \%, 97,2 \%]$} \\
\hline 12 & 27 & $35,1 \%$ & 50 & $64,9 \%$ & 77 & {$[53,8 \%, 74,7 \%]$} \\
\hline 13 & & $3,9 \%$ & 74 & $96,1 \%$ & 77 & {$[89,2 \%, 98,7 \%]$} \\
\hline 14 & & $0,0 \%$ & 77 & $100,0 \%$ & 77 & {$[95,2 \%, 100,0 \%]$} \\
\hline 15 & 4 & $5,2 \%$ & 73 & $94,8 \%$ & 77 & {$[87,4 \%, 98,0 \%]$} \\
\hline 16 & 10 & $13,0 \%$ & 67 & $87,0 \%$ & 77 & {$[77,7 \%, 92,8 \%]$} \\
\hline 17 & 3 & $3,9 \%$ & 74 & $96,1 \%$ & 77 & {$[89,2 \%, 98,7 \%]$} \\
\hline 18 & 6 & $7,8 \%$ & 71 & $92,2 \%$ & 77 & {$[84,0 \%, 96,4 \%]$} \\
\hline 19 & 29 & $37,7 \%$ & 48 & $62,3 \%$ & 77 & {$[51,2 \%, 72,3 \%]$} \\
\hline
\end{tabular}




\subsubsection{Vergleich des Farbsehens mittels Buch und iPad2® bei den Probanden mit Farbsehstörung}

Die Übereinstimmung beim Vergleich der 19 Velhagen/Broschmann/ Kuchenbecker-Farbtafeln zwischen Buch und iPad $2 \AA$ bei den Probanden mit Farbsehstörungen betrug 83,3\% (Tab. 6). Das 95\%-Konfidenzintervall reicht von 78,4 bis $87,3 \%$.

Tab. 6: Vergleich des Farbsehens mittels Buch und $\mathrm{iPad} 2 \AA$ bei den Probanden mit Farbsehstörung

\begin{tabular}{|c|c|c|c|c|c|}
\hline & & Häufigkeit & Prozent & $\begin{array}{l}\text { Gültige } \\
\text { Prozente }\end{array}$ & $\begin{array}{l}\text { Kumulierte } \\
\text { Prozente }\end{array}$ \\
\hline \multirow{3}{*}{ Gültig } & keine Übereinstimmung & 45 & 16,7 & 16,7 & 16,7 \\
\hline & Übereinstimmung (Buch = iPad2®) & 225 & 83,3 & 83,3 & 100,0 \\
\hline & Gesamt & 270 & 100,0 & 100,0 & \\
\hline
\end{tabular}

Die höchste Übereinstimmung besteht mit 100\% bei Tafel 2 (Zeichen: 182), Tafel 5 (Zeichen: 3), Tafel 14 (Zeichen: 49) und Tafel 15 (Zeichen: 5E). Die geringste Übereinstimmung besteht mit 53,3\% bei der Tafel 9 (Zeichen: 6) (Tab. 7). 
Tab. 7: Auswertung der Ergebnisse pro Tafel mittels Buch und iPad2® bei den Probanden mit Farbsehstörungen

\begin{tabular}{|c|c|c|c|c|c|c|}
\hline & $\begin{array}{l}\text { Keine } \\
\text { Übereinstimmung }\end{array}$ & $(\%)$ & Übereinstimmung & $(\%)$ & Gesamt & $\begin{array}{l}95 \%- \\
\text { Konfidenzintervall }\end{array}$ \\
\hline 2 & 0 & $0,0 \%$ & 15 & $100,0 \%$ & 15 & {$[79,6 \%, 100,0 \%]$} \\
\hline 3 & 2 & $13,3 \%$ & 13 & $86,7 \%$ & 15 & {$[62,1 \%, 96,3 \%]$} \\
\hline 4 & 4 & $26,7 \%$ & 11 & $73,3 \%$ & 15 & {$[48,0 \%, 89,1 \%]$} \\
\hline 5 & 0 & $0,0 \%$ & 15 & $100,0 \%$ & 15 & {$[79,6 \%, 100,0 \%]$} \\
\hline 6 & 4 & $26,7 \%$ & 11 & $73,3 \%$ & 15 & {$[48,0 \%, 89,1 \%]$} \\
\hline 7 & 2 & $13,3 \%$ & 13 & $86,7 \%$ & 15 & {$[62,1 \%, 96,3 \%]$} \\
\hline 8 & 2 & $13,3 \%$ & 13 & $86,7 \%$ & 15 & {$[62,1 \%, 96,3 \%]$} \\
\hline 9 & 7 & $46,7 \%$ & 8 & $53,3 \%$ & 15 & {$[30,1 \%, 75,2 \%]$} \\
\hline 10 & & $13,3 \%$ & 13 & $86,7 \%$ & 15 & {$[62,1 \%, 96,3 \%]$} \\
\hline Tafel & & & & & & \\
\hline 112 & & $13,3 \%$ & 13 & $86,7 \%$ & 15 & {$[62,1 \%, 96,3 \%]$} \\
\hline 125 & & $33,3 \%$ & 10 & $66,7 \%$ & 15 & {$[41,7 \%, 84,8 \%]$} \\
\hline 13 & & $20,0 \%$ & 12 & $80,0 \%$ & 15 & {$[54,8 \%, 93,0 \%]$} \\
\hline 14 & & $0,0 \%$ & 15 & $100,0 \%$ & 15 & {$[79,6 \%, 100,0 \%]$} \\
\hline 15 & & $0,0 \%$ & 15 & $100,0 \%$ & 15 & {$[79,6 \%, 100,0 \%]$} \\
\hline 162 & & $13,3 \%$ & & $86,7 \%$ & 15 & {$[62,1 \%, 96,3 \%]$} \\
\hline 17 & & $13,3 \%$ & & $86,7 \%$ & 15 & {$[89,2 \%, 98,7 \%]$} \\
\hline 18 & & $33,3 \%$ & 10 & $66,7 \%$ & 15 & {$[41,7 \%, 84,8 \%]$} \\
\hline 193 & & $20,0 \%$ & 12 & $80,0 \%$ & 15 & {$[54,8 \%, 93,0 \%]$} \\
\hline
\end{tabular}




\subsubsection{Vergleich des Farbsehens zwischen Buch und iPad2® bei den Probanden ohne Farbsehstörung}

Die Übereinstimmung beim Vergleich der 19 Velhagen/Broschmann/ Kuchenbecker-Farbtafeln mittels Buch und $\mathrm{iPad} 2 \AA$ bei den Probanden ohne Farbsehstörungen betrug 89,1\% (Tab. 8). Das 95\%-Konfidenzintervall reicht von 87,1 bis $90,8 \%$.

Tab. 8: Vergleich der Untersuchung des Farbsehens zwischen Buch und iPad $2 \AA$ bei den Probanden ohne Farbsehstörungen

\begin{tabular}{lllll}
\hline \hline & Häufigkeit Prozent $\begin{array}{l}\text { Gültige } \\
\text { Prozente }\end{array}$ & $\begin{array}{l}\text { Kumulierte } \\
\text { Prozente }\end{array}$ \\
\hline keine Übereinstimmung & 122 & 10,9 & 10,9 & 10,9 \\
\multirow{2}{*}{$\begin{array}{l}\text { Überltig } \\
\text { (Buch=iPad2®) }\end{array}$} & 994 & 89,1 & 89,1 & 100,0 \\
Gesamt & 1116 & 100,0 & 100,0 & \\
\hline \hline
\end{tabular}

Die höchste Übereinstimmung besteht mit $100 \%$ bei Tafel 3 (Zeichen: 69), Tafel 10 (Zeichen: 51), Tafel 13 (Zeichen: 42) und Tafel 14 (Zeichen: 49). Die geringste Übereinstimmung besteht mit $51,6 \%$ bei der Tafel 4 (Zeichen: H5) und mit 58,1\% bei Tafel 19 (Zeichen: B5) (Tab. 9). 
Tab. 9: Auswertung der Ergebnisse pro Tafel mittels Buch und iPad2® bei den Probanden ohne Farbsehstörungen

\begin{tabular}{|c|c|c|c|c|c|c|}
\hline & $\begin{array}{l}\text { Keine } \\
\text { Übereinstimmung }\end{array}$ & $(\%)$ & Übereinstimmung & $(\%)$ & Gesamt & $\begin{array}{l}95 \%- \\
\text { Konfidenzintervall }\end{array}$ \\
\hline 2 & 12 & $19,4 \%$ & 650 & $80,6 \%$ & 62 & {$[69,1 \%, 88,6 \%]$} \\
\hline 3 & 0 & $0,0 \%$ & 62 & $100,0 \%$ & 62 & {$[94,2 \%, 100,0 \%]$} \\
\hline 4 & 30 & $48,4 \%$ & 632 & $51,6 \%$ & 62 & {$[39,4 \%, 63,6 \%]$} \\
\hline 5 & 1 & $1,6 \%$ & 61 & $98,4 \%$ & 62 & {$[91,4 \%, 99,7 \%]$} \\
\hline 6 & 4 & $6,5 \%$ & 58 & $93,5 \%$ & 62 & [84,6\%, 97,5\%] \\
\hline 7 & 1 & $1,6 \%$ & 61 & $98,4 \%$ & 62 & {$[91,4 \%, 99,7 \%]$} \\
\hline 8 & 1 & $1,6 \%$ & 61 & $98,4 \%$ & 62 & {$[91,4 \%, 99,7 \%]$} \\
\hline 9 & 8 & $12,9 \%$ & 654 & $87,1 \%$ & 62 & {$[76,6 \%, 93,3 \%]$} \\
\hline 10 & 0 & $0,0 \%$ & 62 & $100,0 \%$ & $\% 62$ & {$[94,2 \%, 100,0 \%]$} \\
\hline Tafel & & & & & & \\
\hline 11 & 3 & $4,8 \%$ & 59 & $95,2 \%$ & 62 & {$[86,7 \%, 98,3 \%]$} \\
\hline 12 & 22 & $35,5 \%$ & 640 & $64,5 \%$ & 62 & {$[52,1 \%, 75,3 \%]$} \\
\hline 13 & 0 & $0,0 \%$ & 62 & $100,0 \%$ & $\% 62$ & {$[94,2 \%, 100,0 \%]$} \\
\hline 14 & 0 & $0,0 \%$ & 62 & $100,0 \%$ & 662 & {$[94,2 \%, 100,0 \%]$} \\
\hline 15 & 4 & $6,5 \%$ & 58 & $93,5 \%$ & 62 & {$[84,6 \%, 97,5 \%]$} \\
\hline 16 & 8 & $12,9 \%$ & 654 & $87,1 \%$ & 62 & {$[76,6 \%, 93,3 \%]$} \\
\hline 17 & 1 & $1,6 \%$ & 61 & $98,4 \%$ & 62 & {$[91,4 \%, 99,7 \%]$} \\
\hline 18 & 1 & $1,6 \%$ & 61 & $98,4 \%$ & 62 & {$[91,4 \%, 99,7 \%]$} \\
\hline 19 & 26 & $41,9 \%$ & 636 & $58,1 \%$ & 62 & {$[45,7 \%, 69,5 \%]$} \\
\hline
\end{tabular}




\subsubsection{Vergleich des Farbsehens mittels iPad1® und iPad2® bei allen Probanden}

Die Übereinstimmung beim Vergleich der 19 Velhagen/Broschmann/ Kuchenbecker-Farbtafeln zwischen iPad1 $\AA$ und iPad $2 \AA$ bei allen Probanden betrug $87,2 \%$ (Tab. 10). Das 95\%-Konfidenzintervall reicht von 81,6 bis $91,3 \%$.

Tab. 10: Vergleich der Untersuchung des Farbsehens mittels iPad1® und iPad2® bei allen Probanden

\begin{tabular}{lllll}
\hline \hline & Häufigkeit & $\begin{array}{l}\text { Prozent } \\
\text { Gültige } \\
\text { Prozente }\end{array}$ & $\begin{array}{l}\text { Kumulierte } \\
\text { Prozente }\end{array}$ \\
\hline keine Übereinstimmung & 23 & 12,8 & 12,8 & 12,8 \\
Gültig $\begin{array}{l}\text { Übereinstimmung } \\
\text { (iPad1®=iPad2®) }\end{array}$ & 157 & 87,2 & 87,2 & 100,0 \\
Gesamt & 180 & 100,0 & 100,0 & \\
\hline \hline
\end{tabular}

Bei den meisten Tafeln besteht eine Übereinstimmung von 90\%-100\%. Die geringste Übereinstimmung besteht mit $30 \%$ bei der Tafel 4 (Zeichen: H5), mit $50 \%$ bei der Tafel 19 (Zeichen: B5) und mit 60\% bei Tafel 2 (Zeichen: 182) und Tafel 12 (Zeichen: CH) (Tab. 11). 
Tab. 11: Auswertung der Ergebnisse pro Tafel mittels iPad1® und iPad2® bei allen Nutzern

\begin{tabular}{|c|c|c|c|c|c|c|}
\hline & $\begin{array}{l}\text { Keine } \\
\text { Übereinstimmung }\end{array}$ & $(\%)$ & Übereinstimmung & $(\%)$ & Gesamt & $\begin{array}{l}95 \%- \\
\text { Konfidenzintervall }\end{array}$ \\
\hline 2 & 4 & \multicolumn{2}{|l|}{$40,0 \% 6$} & $60,0 \%$ & 10 & {$[31,3 \%, 83,2 \%]$} \\
\hline 3 & 1 & \multicolumn{2}{|l|}{$10,0 \% 9$} & $90,0 \%$ & 10 & {$[59,6 \%, 98,2 \%]$} \\
\hline 4 & 7 & \multicolumn{2}{|c|}{$70,0 \% 3$} & $30,0 \%$ & 10 & {$[10,8 \%, 60,3 \%]$} \\
\hline 5 & 0 & \begin{tabular}{l|l}
$0,0 \%$ & 1
\end{tabular} & 10 & $100,0 \%$ & 10 & {$[72,2 \%, 100,0 \%]$} \\
\hline 6 & 0 & $0,0 \% \quad 1$ & 10 & $100,0 \%$ & 10 & {$[72,2 \%, 100,0 \%]$} \\
\hline 7 & 0 & \begin{tabular}{l|l}
$0,0 \%$ & 1
\end{tabular} & 10 & $100,0 \%$ & 10 & {$[72,2 \%, 100,0 \%]$} \\
\hline 8 & 1 & \multicolumn{2}{|l|}{$10,0 \% 9$} & $90,0 \%$ & 10 & {$[59,6 \%, 98,2 \%]$} \\
\hline 9 & 0 & $0,0 \% \quad 1$ & 10 & $100,0 \%$ & 10 & {$[72,2 \%, 100,0 \%]$} \\
\hline 10 & 0 & $0,0 \% \quad 1$ & 10 & $100,0 \%$ & 10 & {$[72,2 \%, 100,0 \%]$} \\
\hline Tafel & & & & & & \\
\hline 11 & 0 & $0,0 \% \quad 1$ & 10 & $100,0 \%$ & 10 & {$[72,2 \%, 100,0 \%]$} \\
\hline 12 & 4 & $40,0 \% 6$ & 6 & $60,0 \%$ & 10 & {$[31,3 \%, 83,2 \%]$} \\
\hline 13 & 0 & $0,0 \% \quad 1$ & 10 & $100,0 \%$ & 10 & {$[72,2 \%, 100,0 \%]$} \\
\hline 14 & 0 & $0,0 \% \quad 1$ & 10 & $100,0 \%$ & 10 & {$[72,2 \%, 100,0 \%]$} \\
\hline 15 & 0 & $0,0 \% \quad 1$ & 10 & $100,0 \%$ & 10 & {$[72,2 \%, 100,0 \%]$} \\
\hline 16 & 0 & $0,0 \%$ & 10 & $100,0 \%$ & 10 & {$[72,2 \%, 100,0 \%]$} \\
\hline 17 & 1 & $10,0 \% \varsigma$ & 9 & $90,0 \%$ & 10 & {$[59,6 \%, 98,2 \%]$} \\
\hline 18 & 0 & $0,0 \%$ & 10 & $100,0 \%$ & 10 & {$[72,2 \%, 100,0 \%]$} \\
\hline 19 & 5 & $50,0 \% 5$ & 5 & $50,0 \%$ & 10 & {$[16,8 \%, 68,7 \%]$} \\
\hline
\end{tabular}




\subsubsection{Vergleich bei der Untersuchung des Farbsehens zwischen Buch und iPad1® bei allen Probanden}

Die Übereinstimmung beim Vergleich der 19 Velhagen/Broschmann/ Kuchenbecker-Farbtafeln zwischen Buch und iPad1® bei allen 10 Probanden, die mittels iPad1® untersucht wurden, betrug $84,4 \%$ (Tab. 12). Das 95\%Konfidenzintervall reicht von 78,4 bis $89,0 \%$.

Tab. 12: Vergleich des Farbsehens zwischen Buch und iPad1® bei allen 10 Probanden, die mittels iPad1® untersucht wurden

\begin{tabular}{|c|c|c|c|c|c|}
\hline & & Häufigkeit & Prozent & $\begin{array}{l}\text { Gültige } \\
\text { Prozente }\end{array}$ & $\begin{array}{l}\text { Kumulierte } \\
\text { Prozente }\end{array}$ \\
\hline \multirow{3}{*}{ Gültig } & keine Übereinstimmung & 28 & 15,6 & 15,6 & 15,6 \\
\hline & $\begin{array}{l}\text { Übereinstimmung } \\
\text { (Buch=iPad1®) }\end{array}$ & 152 & 84,4 & 84,4 & 100,0 \\
\hline & Gesamt & 180 & 100,0 & 100,0 & \\
\hline
\end{tabular}

Bei den meisten Tafeln besteht eine Übereinstimmung von 90\%-100\%. Die geringste Übereinstimmung besteht mit $10 \%$ bei der Tafel 4 (Zeichen: H5), mit 40\% bei der Tafel 19 (Zeichen: B5) und mit 60\% bei Tafel 2 (Zeichen: 182) und Tafel 12 (Zeichen: $\mathrm{CH}$ ) (Tab. 13). 
Tab. 13: Auswertung der Ergebnisse pro Tafel mittels Buch und iPad1® bei allen Nutzern, die mittels iPad1® untersucht wurden

\begin{tabular}{|c|c|c|c|c|c|c|}
\hline & $\begin{array}{l}\text { Keine } \\
\text { Übereinstimmung }\end{array}$ & $(\%)$ & Übereinstimmung & $(\%)$ & Gesamt & $\begin{array}{l}95 \%- \\
\text { Konfidenzintervall }\end{array}$ \\
\hline 2 & 4 & $40,0 \%$ & & $60,0 \%$ & 10 & {$[31,3 \%, 83,2 \%]$} \\
\hline 3 & 1 & $10,0 \%$ & & $90,0 \%$ & 10 & {$[59,6 \%, 98,2 \%]$} \\
\hline 4 & 9 & $90,0 \%$ & & $10,0 \%$ & 10 & {$[1,8 \%, 40,4 \%]$} \\
\hline 5 & 0 & $0,0 \%$ & 10 & $100,0 \%$ & 10 & {$[72,7 \%, 100,0 \%]$} \\
\hline 6 & 1 & $10,0 \%$ & 9 & $90,0 \%$ & 10 & {$[59,6 \%, 98,2 \%]$} \\
\hline 7 & 0 & $0,0 \%$ & 10 & $100,0 \%$ & 10 & {$[72,7 \%, 100,0 \%]$} \\
\hline 8 & 0 & $0,0 \%$ & 10 & $100,0 \%$ & 10 & {$[72,7 \%, 100,0 \%]$} \\
\hline 9 & 0 & $0,0 \%$ & 10 & $100,0 \%$ & 10 & {$[72,7 \%, 100,0 \%]$} \\
\hline 100 & & $0,0 \%$ & 10 & $100,0 \%$ & 10 & {$[72,7 \%, 100,0 \%]$} \\
\hline Tafel & & & & & & \\
\hline $11 \mathrm{C}$ & & $0,0 \%$ & 10 & $100,0 \%$ & 10 & {$[72,7 \%, 100,0 \%]$} \\
\hline $12^{4}$ & 4 & $40,0 \%$ & 6 & $60,0 \%$ & 10 & {$[31,3 \%, 83,2 \%]$} \\
\hline 131 & & $10,0 \%$ & 9 & $90,0 \%$ & 10 & {$[59,6 \%, 98,2 \%]$} \\
\hline 140 & & $0,0 \%$ & 10 & $100,0 \%$ & 10 & {$[72,7 \%, 100,0 \%]$} \\
\hline 150 & & $0,0 \%$ & 10 & $100,0 \%$ & 10 & {$[72,7 \%, 100,0 \%]$} \\
\hline 161 & & $10,0 \%$ & 9 & $90,0 \%$ & 10 & {$[59,6 \%, 98,2 \%]$} \\
\hline 171 & & $10,0 \%$ & 9 & $90,0 \%$ & 10 & {$[59,6 \%, 98,2 \%]$} \\
\hline 180 & & $0,0 \%$ & 10 & $100,0 \%$ & 10 & {$[72,7 \%, 100,0 \%]$} \\
\hline 196 & & $60,0 \%$ & 4 & $40,0 \%$ & 10 & {$[16,8 \%, 68,7 \%]$} \\
\hline
\end{tabular}




\subsubsection{Vergleich bei der Untersuchung des Farbsehens zwischen den drei Gruppen (Buch vs. iPad1®, Buch vs. iPad2®, iPad1® vs. iPad2®)}

Wenn man einen Gesamtvergleich zwischen den drei Gruppen (Buch vs. iPad $1 \AA$, Buch vs. iPad $2 \AA$, iPad $1 \AA$ vs. iPad $2 \AA)$ anstellt, so zeigen sich folgende Übereinstimmungen:

- Buch vs. iPad2®: $1219 / 1386=88,0 \%$

- Buch vs. iPad1®: 152/180 = 84,4\%

- iPad1® vs. iPad2®: $157 / 180=87,2 \%$

Der $p$-Wert nach Fisher-Freeman-Haltons exaktem Kontingenztafeltest beträgt 0,375. Also zeigen sich zwischen den drei Gruppen keine signifikanten Unterschiede.

Die Übereinstimmung zwischen den Tafeln bei den drei Gruppen nach Landis und Koch wird nach Bestimmung des Cohen's Kappa Wertes (K) (s. Anhang 3) in der Tabelle 14 als Übersicht dargestellt (Landis et al., 1977). 
Tab. 14: Übereinstimmung nach Landis und Koch zwischen den drei Gruppen (Buch vs. iPad2®, Buch vs. iPad $1 \AA$, iPad $1 \AA$ vs. iPad $2 \AA), k<0=$ „schlechte Übereinstimmung“, $0<\mathrm{k}<0,2=$ „etwas Übereinstimmung“, $\quad 0,21<\mathrm{k}<0,40=$ "ausreichende Übereinstimmung“, $0,41<\mathrm{k}<0,60=$ „mittelmäßige (moderate) Übereinstimmung", $0,61<k<0,80=$ „beachtliche Übereinstimmung“, $0,81<\mathrm{k}<1,00=$,vollkommene Übereinstimmung“,n.b.= nicht berechenbar

\begin{tabular}{|l|l|l|l|l|l|}
\hline \multicolumn{2}{|l|}{$\begin{array}{l}\text { Buch vs. } \\
\text { iPad2 }\end{array}$} & \multicolumn{2}{l|}{$\begin{array}{l}\text { Buch vs. } \\
\text { iPad1® }\end{array}$} & $\begin{array}{l}\text { iPad1 } \\
\text { vs. } \\
\text { iPad2 }\end{array}$ \\
\hline Tafel & Alle & Farbfehlsichtige & Farbnormale & Alle & Alle \\
\hline 2 & b & v & b & v & e \\
\hline 3 & v & v & n.b. & m & n.b. \\
\hline 4 & m & a & m & a & a \\
\hline 5 & v & v & v & v & e \\
\hline 6 & b & m & m & b & v \\
\hline 7 & v & b & v & v & e \\
\hline 8 & v & b & n.b. & b & b \\
\hline 9 & b & e & b & v & e \\
\hline 10 & v & b & n.b. & v & v \\
\hline 11 & v & m & v & v & e \\
\hline 12 & m & m & a & m & n.b. \\
\hline 13 & b & b & n.b. & n.b & n.b. \\
\hline 14 & v & v & n.b. & n.b & n.b. \\
\hline 15 & m & n.b. & a & v & v \\
\hline 16 & b & n.b. & e & b & b \\
\hline 17 & v & b & b & v & b \\
\hline 18 & b & a & b & v & v \\
\hline 19 & m & b & m & m & a \\
\hline
\end{tabular}




\section{Diskussion}

\subsection{Probanden}

Insgesamt wurden 77 Probanden untersucht. Die Anzahl der Probanden kann möglicherweise die Aussagekraft der Ergebnisse beeinflussen. Von unseren Probanden hatten 15 männliche Probanden eine angeborene Farbsehstörung. Die hohe Anzahl (ca. 20\%) an Probanden mit Farbsehstörungen ist darauf zurückzuführen, dass diese Probanden gezielt ausgesucht wurden. Ansonsten wären nur 8\% der Probanden mit Farbsehstörungen zu erwarten (Birch, 2012; Deeb et al., 1993; Krastel, 1995).

Im Rahmen dieser Studie wurde bei keinem Probanden eine Blau-Gelb-Störung nachgewiesen. Dies liegt daran, dass angeborene Tritanen mit einer Häufigkeit von 1:10000 bis 1:60000 sehr selten sind (Kalmus, 1955a).

Außerdem wurden keine Patienten mit erworbenen Farbsehstörungen untersucht. Bei dem niedrigen Durchschnittsalter von 42,81 \pm 16,95 Jahre ist keine erworbene Erkrankung zu erwarten. Bei den älteren Probanden ist keine Funduskopie z. A. einer Makuladegeneration bzw. Optikusatrophie durchgeführt worden. Diese sind aber bei den sehr guten Nahvisusergebnissen eher unwahrscheinlich.

\subsection{Bewertung der Tafeln}

Tafel 1: Zeichen $2 \mathrm{~L}$

Diese Demonstrationstafel wurde stets von allen Probanden sowohl mittels Buch als auch mittels iPad® erkannt.

Tafel 2: Zeichen 182

Diese diagnostische Tafel wurde sowohl bei den Farbnormalen als auch bei den Farbfehlsichtigen bei der Untersuchung des Farbsehens mittels Buch, iPad1® und iPad $2 \AA$ oft falsch erkannt. Die möglichen Angaben waren 8, 132 oder 82. 
Bei den Probanden mit Farbsehstörung wurde hauptsächlich nur die Zahl 8 erkannt, was für eine Rot-Grün-Störung sprechen könnte. Die Übereinstimmung bei allen Probanden zwischen Buch und iPad1® sowie zwischen iPad1® und iPad $2 \AA$ war geringer als zwischen Buch und $\mathrm{iPad} 2 \AA$, was auf eine schlechte Farbdarstellung bei iPad1® zurückzuführen sein könnte.

Tafel 3: Zeichen 69

Bei dieser Tafel mit alternativem Sehzeichen zur Detektion einer Rot-GrünStörung zeigte sich bei der Untersuchung des Farbsehens mittels Buch und iPad $2 \AA$ eine gute Übereinstimmung. Zwischen Buch und iPad1® zeigte sich eine moderate Übereinstimmung, was möglicherweise auf die unterschiedliche Farbraumdarstellung zwischen den verschiedenen Tablet-PC-Modellen zurückzuführen ist.

Tafel 4: Zeichen H5

Diese Tafel mit verschwindenden Sehzeichen zur Detektion einer Rot-GrünStörung wurde bei allen Probanden, den Farbnormalen und den Farbfehlsichtigen, bei der Untersuchung der Farbsehens mittels Buch, iPad1® und iPad $2 \AA$ häufig falsch erkannt, sodass diese Tafel als nicht gut geeignet für die Untersuchung des Farbsehens, weder mittels Buch, noch mittels iPad®, anzusehen ist. Häufige falsche Angaben waren N5, HS, NS, X5, X.

Tafel 5: Zeichen 3

Diese Tafel mit verschwindenden Sehzeichen zur Feststellung einer Rot-GrünStörung wurde immer richtig in allen Gruppen der Farbnormalen erkannt. Bei den Farbfehlsichtigen wurde eine hohe Übereinstimmung zwischen Buch und iPad $2 \circledast$ erzielt, sodass diese Tafel als geeignet für die Untersuchung mittels Tablet-PC anzusehen ist. Zwischen iPad1® und $\mathrm{iPad} 2 \AA$ zeigte sich eine schlechte Übereinstimmung, was wahrscheinlich an der unterschiedlichen Farbdarstellung der verschiedenen iPad-Modelle liegt. 
Tafel 6: Zeichen 6R

Diese Tafel mit verschwindenden Sehzeichen zur Detektion einer Rot-GrünStörung zeigte sich zwischen Buch und $\mathrm{iPad} 2 \AA$ bei allen Probanden eine geringe Übereinstimmung, was wahrscheinlich auf technische Limitationen der Farbdarstellung zurückzuführen ist.

\section{Tafel 7: Zeichen 2}

Diese Tafel mit verschwindenden Sehzeichen zur Detektion einer Rot-GrünStörung zeigte bei den Farbfehlsichtigen zwischen Buch und $\mathrm{Pad} 2 \AA$ eine etwas geringere Übereinstimmung, was auf technische Limitationen der Farbdarstellung zurückzuführen sein könnte. Es zeigte sich auch eine deutlich geringe Übereinstimmung zwischen $\mathrm{iPad} 1 \AA$ und $\mathrm{iPad} 2 \AA$. Dies könnte durch die unterschiedliche Farbdarstellung zwischen diesen iPad-Versionen bedingt sein.

\section{Tafel 8: Zeichen F4}

Diese Tafel mit verschwindenden Sehzeichen zur Detektion einer Rot-GrünStörung zeigte in allen Gruppen eine hohe Übereinstimmung. Diese Tafel ist daher als eine geeignete Tafel für die Untersuchung des Farbsehens auch mittels Tablet-PC anzusehen.

Tafel 9: Zeichen 6

Diese Tafel mit verschwindenden Sehzeichen zur Feststellung einer Rot-GrünStörung zeigte bei den Farbnormalen eine relativ gute Übereinstimmung. Bei den Farbfehlsichtigen zeigte sich zwischen Buch und $\mathrm{iPad} 2 \AA$ eine geringere Übereinstimmung, was auf technische Limitationen der Farbdarstellung zurückzuführen sein könnte. Zudem zeigte sich hier auch eine schlechte Übereinstimmung zwischen den beiden iPad-Versionen (iPad1® vs. iPad2®). 
Tafel 10: Zeichen 51

Diese Tafel mit verschwindenden Sehzeichen zur Detektion einer Rot-GrünStörung zeigte bei den Farbnormalen in allen Gruppen eine gute Übereinstimmung. Bei den Farbfehlsichtigen war die Übereinstimmung etwas geringer.

\section{Tafel 11: Zeichen 3}

Diese Tafel mit alternativen Sehzeichen zur Detektion einer Rot-Grün-Störung zeigte bei den Farbnormalen in allen Gruppen eine gute Übereinstimmung. Bei den Farbfehlsichtigen sowie zwischen iPad1® und $\mathrm{iPad} 2 \AA$ zeigte sich eine deutlich geringere Übereinstimmung. Dies könnte durch die technische Limitationen der Farbdarstellung des iPads ${ }^{\circledR}$ bedingt sein.

Tafel 12: Zeichen $\mathrm{CH}$

Diese Tafel mit alternativen Sehzeichen zur Detektion einer Rot-Grün-Störung wurde bei allen drei Untersuchungsmethoden oft falsch erkannt und zeigte geringere Übereinstimmungen. Die möglichen Angaben waren $6 \mathrm{H}, \mathrm{GH}, \mathrm{C7}, \mathrm{OH}$. Man kann davon ausgehen, dass diese Tafel für die Untersuchung des Farbsehens generell nicht gut geeignet ist.

Tafel 13: Zeichen 42

Diese Tafel mit verschwindenden Zeichen zur Detektion von Rot-Grün-Störungen zeigte bei allen Gruppen eine relativ hohe Übereinstimmung. Daher ist diese Tafel als eine gut geeignete Tafel für die Untersuchung des Farnsehens mittels Tablet-PC anzusehen.

\section{Tafel 14: Zeichen 49}

Diese Tafel mit verschwindenden Zeichen dient zur Detektion einer Blau-GelbStörung. Diese Tafel wurde bei allen Untersuchungsmitteln ähnlich erkannt, sodass eine hohe Übereinstimmung zu verzeichnen war. Ob sie zur Detektion 
einer Blau-Gelb-Störung geeignet ist, kann nicht beurteilt werden, da keine Probanden mit Blau-Gelb-Störung vorhanden waren.

Tafel 15: Zeichen 5E

Diese Tafel mit verschwindenden Zeichen zur Detektion einer Blau-Gelb-Störung wurde von den Farbnormalen in einigen Fällen falsch erkannt. Die möglichen Angaben waren $6 \mathrm{E}, 56,52$. So ist diese Blau-Gelb-Tafel der Blau-Gelb-Tafel Nr. 14 etwas unterlegen. Dies ist auf die schlechte Konstruktion der Tafel zurückzuführen, wie bei Kuchenbecker et al. angemerkt ist (Kuchenbecker, Nicklas, et al., 2010).

Tafel 16: Zeichen 65

Diese Tafel mit verschwindenden Sehzeichen zur Detektion einer Rot-GrünStörung zeigte bei den Farbnormalen zwischen Buch und iPad $2 \AA$ eine geringe Übereinstimmung. Bei den kleinen Gruppen (Buch vs. $\mathrm{iPad} 1 \AA$ und $\mathrm{iPad} 1 \AA$ vs. iPad2®) bestand eine relativ hohe Übereinstimmung.

Tafel 17: Zeichen RG

Diese Tafel mit verschwindenden Sehzeichen zur Detektion von Rot-GrünStörungen zeigte mit allen drei Untersuchungsmitteln bei den Farbnormalen und den Farbfehlsichtigen eine hohe Übereinstimmung, sodass sie als gut geeignet für die Untersuchung des Farbsehens mittels Tablet-PC anzusehen ist.

Tafel 18: Zeichen A4

Diese Tafel mit verschwindenden Sehzeichen zeigte bei den Farbnormalen eine relativ gute Übereinstimmung. Bei den Farbfehlsichtigen zeigte sich zwischen Buch und $\mathrm{iPad} 2 \AA$ eine geringere Übereinstimmung, was auf technische Limitationen der Farbdarstellung zurückzuführen sein könnte. 
Tafel 19: Zeichen B5

Diese Tafel mit alternativen Zeichen zur Detektion von Rot-Grün-Störungen zeigte in allen Fällen eine schlechte Übereinstimmung, sodass sie für die Untersuchung des Farbsehens generell nicht so gut geeignet ist.

Alle Probanden

Bei der Untersuchung des Farbsehens mittels Buch und $\mathrm{iPad} 2 \AA$ bei allen Probanden bestand die niedrigste Übereinstimmung mit 55,8\% bei der Tafel 4 (Zeichen: H5). Darauf folgt bei Tafel 19 (Zeichen: B5), Tafel 12 (Zeichen: $\mathrm{CH}$ ), Tafel 9 (Zeichen: 6) und Tafel 2 (Zeichen: 182) die nächstniedrigere Übereinstimmung. Daher sind diese Tafeln eher als nicht gut geeignet für die Untersuchung des Farbsehens mittels Tablet-PC (iPad2®) anzusehen. Die höchste Übereinstimmung zeigte mit 100\% die Blau-Gelb-Tafel 14 (Zeichen: 49). Dies ist dadurch erklärt, dass bei keinem Probanden eine Blau-Gelb-Störung vorlag.

\section{Probanden mit Farbsehstörung}

Bei der Untersuchung des Farbsehens mittels Buch und $\mathrm{iPad} 2 \AA$ bei den Probanden mit Farbsehstörung zeigte die Rot-Grün-Tafel 9 (Zeichen: 6) mit 53,3\% die niedrigste Übereinstimmung. Die Tafeln 12 (Zeichen: $\mathrm{CH}$ ) und 18 (Zeichen: A4), ebenfalls Tafeln zur Detektion einer Rot-Grün-Störung zeigten mit 66,7\% die nächstniedrigere Übereinstimmung. Die Tafeln 4 (Zeichen: H5) und 6 (Zeichen: 6R) gelten mit einer Übereinstimmung von $73,3 \%$ auch als nicht gut geeignet für die Untersuchung mittels Tablet-PC (iPad2®). Hingegen zeigten die Tafel 2 (Zeichen: 182), Tafel 5 (Zeichen: 3), Tafel 14 (Zeichen: 49) und Tafel 15 (Zeichen: 5E) eine Übereinstimmung von 100\%, sodass bei diesen Tafeln eine Untersuchung des Farbsehens mittels Buch und $\mathrm{iPad}{ }^{\circledR}$ als gleichwertig anzusehen ist. Bei der Einordnung der Übereinstimmung nach Landis und Koch zeigt sich das iPad2® bei den Tafeln 6 (Zeichen: 6R), 9 (Zeichen: 6), 11 (Zeichen: 3) und 18 (Zeichen: A4) bei den Probanden mit Farbsehstörungen gegenüber dem Buch unterlegen. 
Probanden ohne Farbsehstörung

Bei der Untersuchung des Farbsehens mittels Buch und iPad2 ${ }^{\circledR}$ bei den Probanden ohne Farbsehstörungen zeigten die Tafel 4 (Zeichen: H5), die Tafel 19 (Zeichen: B5) und die Tafel 12 (Zeichen: $\mathrm{CH}$ ) mit jeweils einem Prozentsatz von $51,6 \%, 58,1 \%$ und $64,5 \%$ eine niedrige Übereinstimmung. Dies ist zurückzuführen auf die Verwendung mehrdeutiger Zeichen. Andererseits bestand eine Übereinstimmung von $100 \%$ bei der Tafel 3 (Zeichen: 69), Tafel 10 (Zeichen: 51), Tafel 13 (Zeichen: 42) und Tafel 14 (Zeichen: 49).

Alle Probanden, die mittels iPad1® untersucht wurden

Bei der Untersuchung des Farbsehens bei den Probanden, welche mit dem iPad1® untersucht wurden, zeigten die Tafeln 2 (Zeichen: 182), 4 (Zeichen: H5), 12 (Zeichen: $\mathrm{CH}$ ) und 19 (Zeichen: B5) die niedrigste Übereinstimmung zwischen Buch und $\mathrm{iPad} 1 \AA$ bzw. zwischen $\mathrm{iPad} 1 \AA$ und $\mathrm{iPad} 2 \AA$, während bei den restlichen Farbtafeln eine hohe Übereinstimmung bestand.

Zusammenfassend kann festgestellt werden, dass die Tafeln „182“, „H5“, „CH“ und „B5“ bei allen Gruppen, bei der Untersuchung mit dem Buch als auch bei der Untersuchung mit dem Tablet-PC (iPad1® und $\mathrm{iPad} 2 \AA$ ), sehr oft verwechselt wurden. Somit sind diese Tafeln generell als nicht gut geeignet für die Untersuchung des Farbsehens anzusehen. Auf der anderen Seite wurden die Tafeln „69“, „3“ (Tafel Nr. 5), „2“, „F4“, „51“, „42“, „49“, „5E“ und „RG“ von allen Nutzern entsprechend ihrer Farbsehfähigkeit erkannt, sodass diese Farbtafeln für die Testung des Farbsehens mittels Tablet-PC gut geeignet sind. Das iPad2® zeigte sich bei den Probanden mit Farbsehstörung insbesondere bei den Tafeln „6R“, „6“, „3“(Tafel Nr. 11) und „A4“ unterlegen.

\subsection{Technische Limitationen}

Unerlässlich ist die Durchführung des Tests unter kontrollierten Untersuchungsbedingungen, mit dem Buch bei Tageslicht und bei der Untersuchung mit dem Tablet-PC bei maximaler Helligkeit des Displays und 70 
cm Abstand. Es sollte auch die jeweils beste mögliche Nahkorrektur verwendet werden. Um die Zuverlässigkeit der Testung des Farbsehens mittels Tablet-PC zu gewährleisten, sollten die Patienten immer darauf hingewiesen werden, die empfohlenen Untersuchungsbedingungen einzuhalten.

Bei der Untersuchung des Farbsehens mit pseudoisochromatischen Tafeln besteht generell das Problem der exakten Farbwiedergabe. Es sind bei verschiedenen Auflagen der Velhagen/Broschmann-Tafeln als auch bei den Ishihara-Tafeln (Adams et al., 1975) Farbunterschiede beschrieben worden, welche auf Fehler bei der Herstellung oder auf das mit der Zeit einsetzende Ausbleichen der Tafeln zurückzuführen sind (Kuchenbecker, Nicklas, et al., 2010; Kuchenbecker, Roehl, et al., 2010).

Beim Tablet-PC spielt die Farbverbindlichkeit eine große Rolle. Das iPhone $\AA$ und das iPad® haben nicht das "color management system" wie ein Apple-Computer. Außerdem weist der Apple-Computer höhere Auflösungen auf. Es bleibt festzuhalten, dass derzeit eine Farbverbindlichkeit bei den Geräten (Tablet-PCs) nicht gegeben ist. Daher sind diese hinsichtlich der Farbqualität nicht so zuverlässig.

Der sRGB-Farbraum ist größer als der des iPads $\AA$, sodass nicht alle Farben auf dem iPad® wiedergegeben werden können.

Auch bei der Untersuchung des Farbsehens mittels Tablet-PC ergeben sich Unterschiede hinsichtlich der Farbwiedergabe zwischen den verschiedenen iPad®-Versionen. Die Farbraumdarstellung ist deutlich unterschiedlich zwischen den verschieden Tablet-PC-Modellen. Das iPad3® kann 66,1\% des Adobe-RGB1998-Farbraums darstellen - eine Verbesserung um fast 35\% gegenüber den vorherigen iPads $\AA^{\circledR}$. Dies wurde allerdings im Rahmen unserer Studie nicht getestet. Es zeigte sich noch, dass das iPad2® hinsichtlich der Farbraumdarstellung geringfügig schlechter als das iPad1® abschneidet. Das iPad3® führt die Liste an, mit deutlichem Abstand zum nächsten Konkurrenten sowie zum iPad2 $\AA$, das nur $68,9 \%$ des sRGB-Farbraums darstellt und damit 
25,5\% hinter dem iPad3® zurückbleibt. Daher zeigte sich, dass das iPad2® hinsichtlich des Prozentsatzes des dargestellten Adobe-RGB-1998- und sRGBFarbraums dem iPad $1 \AA$ und $\mathrm{iPad} 3 \AA$ unterlegen ist. Das $\mathrm{iPad} 3 \AA$ hat außerdem große Fortschritte bei der Darstellung von dunklen Blau-, Magenta- und Rottönen gemacht. Gleiches gilt für blaue und rote Mitteltöne sowie helles Grün. Bemerkenswert ist allerdings, dass die Fähigkeiten des iPad $2 \AA$ bei CyanSchatten und Cyan-Mitteltönen größer sind (M.Wellendorf, 2012). Demzufolge kann es sein, dass die unterschiedlichen Generationen des iPads zu unterschiedlichen Ergebnissen führen könnten. Allerdings wurde in unserer Studie immer der gleiche Tablet-PC (iPad2® bzw. iPad1 $\AA$ ) verwendet.

Ein anderes Problem ist die Vergleichbarkeit zwischen den Tablet-PCs der gleichen Generation. In unserer Studie wurden z. B. nicht zwei iPads2® verglichen. Auch hier könnten Unterschiede in der Farbdarstellung auftreten.

Um die generelle Eignung von Tablet-PCs für die Untersuchung des Farbsehens zu überprüfen, wäre auch der Vergleich zwischen Tablet-PCs mit verschiedenen Betriebssystemen, iOs und Android, möglich, da auch diese Tablet-PCs unterschiedliche Displays besitzen. Allerdings haben wir uns nur auf die Untersuchung mittels iPad $1 \AA$ und $\mathrm{iPad} 2 \AA$ beschränkt. 


\section{Zusammenfassung}

In den letzten Jahren hat die Nutzung von Tablet-PCs im täglichen Alltag stark zugenommen. Es stehen zahlreiche medizinische und ophthalmologische Applikationen zur Verfügung, die ein gutes Hilfsmittel in der klinischen Versorgung der Patienten, der Datenverarbeitung und der Kommunikation sein können. Auch für die Testung des Farbsehens gibt es verschiedene Applikationen, die jedoch bisher in Studien nicht auf ihre Tauglichkeit untersucht worden sind.

Unter kontrollierten Testbedingungen wurde daher in einem Zeitraum von Mai 2012 bis Dezember 2013 ein Farbsehtest mittels Velhagen/Broschmann/Kuchenbecker-Tafeln unter Verwendung eines TabletPCs (iPad2®) durchgeführt. Dabei wurden randomisiert die Tafeln mittels Buch und mittels Tablet-PC (iPad®) präsentiert. Es wurden insgesamt 77 freiwillige Probanden mit einem Durchschnittsalter von 42,81 \pm 16,95 Jahren und einem mittleren Nahvisus von 0,99 $\pm 0,15$ untersucht. 62 Probanden waren farbnormal, und 15 Probanden wiesen eine Farbsehstörung auf. Bei 10 Probanden erfolgte zusätzlich eine Testung mittels iPad1®. Die Farbsehtestung unter Verwendung von 19 Velhagen/Broschmann/Kuchenbecker-Farbtafeln ergab eine Übereinstimmung von $88 \%$ zwischen Buch und Tablet-PC (iPad2 $\AA$ ) bei allen Probanden. Bei den Probanden mit Farbsehstörung bestand eine Übereinstimmung von $83,3 \%$ und bei den Probanden ohne Farbsehstörung eine Übereinstimmung von 89,1\%. Zwischen Buch und iPad1® bestand eine Übereinstimmung von $84,4 \%$ und zwischen iPad1 $\AA$ und $i P a d 2 \AA$ eine Übereinstimmung von $87,2 \%$. Der p-Wert zwischen den drei Gruppen (Buch vs. iPad1®, Buch vs. iPad2 $\AA$, iPad1 ${ }^{\circledR}$ vs. iPad2®) nach Fischer/Freeman/Haltons exaktem Kontingenztafeltest betrug 0,375. Daher zeigten sich zwischen den drei Gruppen keine signifikanten Unterschiede.

Nach den vorliegenden Ergebnissen kann man davon ausgehen, dass unter den durchgeführten Untersuchungsbedingungen Buch und Tablet-PC (iPad2® bzW. 
iPad1®) in etwa vergleichbare Untersuchungsergebnisse liefern, wobei die Trennschärfe des Buches im Vergleich zum iPad2® besser war.

Einige Farbtafeln („182“, „H5“, „CH“ und „B5“) haben sich bei der Untersuchung des Farbsehens sowohl mittels Tablet-PC als auch mittels Buch als nicht besonders gut geeignet erwiesen, da diese Tafeln auch von den Probanden ohne Farbsehstörung oft nicht richtig erkannt wurden. Das iPad2® zeigte sich bei den Probanden mit Farbsehstörung insbesondere bei den Tafeln „6R“, „6“, „3“ (Tafel Nr. 11) und „A4“ unterlegen, was am wahrscheinlichsten auf die technischen Limitationen des Verfahrens zurückzuführen ist. 


\section{Literaturverzeichnis}

Adams, A. J., Balliet, R., \& McAdams, M. (1975). Color vision: blue deficiencies in children? Invest Ophthalmol, 14(8), 620-625.

Aguilera, M. (2010). Farnsworth Munsell 100 Hue test. Retrieved 2 April, 2010, from https://colorcritical.wordpress.com/2010/04/02/farnsworth-munsell100-hue-test/

Apple. (2013a). iPad 2-Technische Daten. Retrieved 18.06.2013, from https://support.apple.com/kb/SP622?viewlocale=de_DE\&locale=de_DE

Apple. (2013b). iPad-Technische Daten. Retrieved 05.08.2013, from https://support.apple.com/kb/SP580?locale=de_DE\&viewlocale=de_DE

Arden, G., Gunduz, K., \& Perry, S. (1988). Color vision testing with a computer graphics system: preliminary results. Doc Ophthalmol, 69(2), 167-174.

Aslam, T. M., et al. (2013). An assessment of a modern touch-screen tablet computer with reference to core physical characteristics necessary for clinical vision testing. J R Soc Interface, 10(84), 20130239. doi: 10.1098/rsif.2013.0239

Atonov, D. I., \& Belichenko, I. A. (1970). [Changes in the colorfield of vision in patients with arterial hypertension]. Klin Med (Mosk), 48(9), 53-60.

Awad, Z., Natt, R. S., \& Pothier, D. D. (2007). Ishihara plates on your handheld computer. Clin Otolaryngol, 32(1), 58-59.

Barsam, A., Bhogal, M., Morris, S., \& Little, B. (2010). Anterior segment slitlamp photography using the iPhone. J Cataract Refract Surg, 36(7), 1240-1241. doi: 10.1016/j.jcrs.2010.04.001

Bayer, A., et al. (1995). [Sensitive physiologic perceptual tests for ocular side effects of drugs exemplified by various anticonvulsants]. Ophthalmologe, 92(2), 182-190.

Behrens-Baumann, W., Prange, H., Ritter, G., Conrad, B., \& Benecke, R. (1981). [Neuro-ophthalmological findings in patients with neurosyphilis (author's transl)]. Nervenarzt, 52(2), 90-93.

Benkert, S. (2012, 04.01.2012). Bilder und Farbmanagement auf dem iPad. from https://extranet.oew.de/blog/2012/01/04/bilder-und-farbmanagement-aufdem-ipad/

Bimler, D., \& Kirkland, J. (2004). Multidimensional scaling of D15 caps: colorvision defects among tobacco smokers? Vis Neurosci, 21(3), 445-448.

Birch, J. (1997a). Clinical use of the City University Test (2nd Edition). Ophthalmic Physiol Opt, 17(6), 466-472.

Birch, J. (1997b). Efficiency of the Ishihara test for identifying red-green colour deficiency. Ophthalmic Physiol Opt, 17(5), 403-408.

Birch, J. (2012). Worldwide prevalence of red-green color deficiency. J Opt Soc Am A Opt Image Sci Vis, 29(3), 313-320. doi: 10.1364/JOSAA.29.000313

Birch, J., Kolle, R. U., Kunkel, M., Paulus, W., \& Upadhyay, P. (1998). Acquired colour deficiency in patients with Parkinson's disease. Vision Res, 38(21), 3421-3426. 
Bockelmann, I., Lindner, H., Peters, B., \& Pfister, E. A. (2003). [Influence of long term occupational exposure to solvents on colour vision]. Ophthalmologe, 100(2), 133-141. doi: 10.1007/s00347-002-0712-z

Boynton, R. M. (1988). Color vision. Annu Rev Psychol, 39, 69-100. doi: 10.1146/annurev.ps.39.020188.000441

Broschmann, D. (2009). [Zusammenstellung der Farbtafeln nach Stilling - Hertel Velhagen - Broschmann (persönl. Mitteilung)].

Carroll, J., Neitz, J., \& Neitz, M. (2002). Estimates of L:M cone ratio from ERG flicker photometry and genetics. J Vis, 2(8), 531-542. doi: 10:1167/2.8.1

Cavanagh, P., Maurer, D., Lewis, T., MacLoad, D. A. I., \& Mather, G. (1986). Computer-generated screening test for color blindness. Color Research, 11, 63-66.

Cheng, N. M., Chakrabarti, R., \& Kam, J. K. (2014). iPhone applications for eye care professionals: a review of current capabilities and concerns. Telemed J E Health, 20(4), 385-387. doi: 10.1089/tmj.2013.0173

Chhablani, J., Kaja, S., \& Shah, V. A. (2012). Smartphones in ophthalmology. Indian J Ophthalmol, 60(2), 127-131. doi: 10.4103/0301-4738.94054

Chiu, C. J., et al. (2014). A risk score for the prediction of advanced age-related macular degeneration: development and validation in 2 prospective cohorts. Ophthalmology, 121(7), 1421-1427. doi: 10.1016/j.ophtha.2014.01.016

Cole, B. (1972). The handicap of abnormal color vision. Aust J Optom, 55:304310.

Colour vision test. (2004). from http://www.leatest.fi/en/vistests/pediatric/cvtests/colortst.html

Computerartikel-Archiv. (2009). RGB-Farbsystem.

Conway, B. R. (2009). Color vision, cones, and color-coding in the cortex. Neuroscientist, 15(3), 274-290.

Crossland, M. D., R, S. S., \& Macedo, A. F. (2014). Smartphone, tablet computer and e-reader use by people with vision impairment. Ophthalmic Physiol Opt, 34(5), 552-557. doi: 10.1111/opo.12136

Dain, S. J. (2004). Clinical colour vision tests. Clin Exp Optom, 87(4-5), 276-293.

Dartnall, H. J., Bowmaker, J. K., \& Mollon, J. D. (1983). Human visual pigments: microspectrophotometric results from the eyes of seven persons. Proc $R$ Soc Lond B Biol Sci, 220(1218), 115-130.

De Valois, R. L., \& Abramov, I. (1966). Color vision. Annu Rev Psychol, 17, $337-$ 362. doi: 10.1146/annurev.ps.17.020166.002005

De Valois, R. L., Abramov, I., \& Mead, W. R. (1967). Single cell analysis of wavelength discrimination at the lateral geniculate nucleus in the macaque. J Neurophysiol, 30(3), 415-433.

Deeb, S. S., \& Motulsky, A. G. (1993). Red-Green Color Vision Defects. In R. A. Pagon, M. P. Adam, H. H. Ardinger, T. D. Bird, C. R. Dolan, C. T. Fong, R. J. H. Smith \& K. Stephens (Eds.), GeneReviews(R). Seattle (WA).

Deeb, S. S., \& Motulsky, A. G. (1996). Molecular genetics of human color vision. Behav Genet, 26(3), 195-207.

Diffen. (2015). Android vs. iOs. from http://www.diffen.com/difference/Android vs iOS 
DIN, -. (2009). Farbmessung-Teil 1: Grundbegriffe der Farbmetrik: Beuth Verlag.

Dortmund, T. U. (1999). Unscharfe Farbverarbeitung, Farbbilder. from http://Irb.cs.uni-dortmund.de/ hildebra/Publications/98lss/Abb5.gif

Edsel, B., Parker, J. A., \& Emerton, L. A. (1994). Computerized color vision testing. Can J Ophthalmol, 29, 125-128.

Erb, C., \& Fahle, M. (2006). [Colour vision and acquired colour vision disturbances. Part I: basic aspects]. Ophthalmologe, 103(4), 349-360; quiz 361. doi: 10.1007/s00347-006-1325-8

Erb, C., et al. (1999). Colour vision disturbances in chronic smokers. Graefes Arch Clin Exp Ophthalmol, 237(5), 377-380.

F. Hollwich, J. F. (1977). Augenheilkunde in Klinik und Praxis (Vol. Band 1). Stuttgart: Georg Thieme Verlag.

Fong, D. S., Barton, F. B., \& Bresnick, G. H. (1999). Impaired color vision associated with diabetic retinopathy: Early Treatment Diabetic Retinopathy Study Report No. 15. Am J Ophthalmol, 128(5), 612-617.

Friendly, D. S., \& Weiss, I. P. (1985). An automated visual acuity testing computer program using the Apple II system. Am J Ophthalmol, 99(2), 188-192.

Frings, S., et al. (1998). Characterization of ether-a-go-go channels present in photoreceptors reveals similarity to IKx, a K+ current in rod inner segments. J Gen Physiol, 111(4), 583-599.

Fusco, G., Tekin, E., Ladner, R. E., \& Coughlan, J. M. (2014). Using Computer Vision to Access Appliance Displays. ASSETS, 2014, 281-282. doi: $10.1145 / 2661334.2661404$

Gegenfurtner, K. R., \& Kiper, D. C. (2003). Color vision. Annu Rev Neurosci, 26, 181-206. doi: 10.1146/annurev.neuro.26.041002.131116

Gegenfurtner, K. R., Walter, S., \& Braun, D. I. (2010). Visuelle Informationsverarbeitung im Gehirn. from http://www.allpsych.unigiessen.de/karl/teach/aka.htm

Gierling, R. (2001). Farbmanagement (1. Auflage ed.). Bonn: mitp-Verlag.

Gouras, P. (1968). Identification of cone mechanisms in monkey ganglion cells. J Physiol, 199(3), 533-547.

Gouras, P. (1969). Antidromic responses of orthodromically identified ganglion cells in monkey retina. J Physiol, 204(2), 407-419.

Gouras, P. (1991). The perception of colour. In J. R. Cronly-Dillon (Ed.), Vision and visual dysfunction (Vol. 6). Basingstoke: Mac-Millan.

Gouras, P. (1995). Color Vision. In H. Kolb, E. Fernandez \& R. Nelson (Eds.), Webvision: The Organization of the Retina and Visual System. Salt Lake City (UT).

Greenstein, V., Sarter, B., Hood, D., Noble, K., \& Carr, R. (1990). Hue discrimination and $S$ cone pathway sensitivity in early diabetic retinopathy. Invest Ophthalmol Vis Sci, 31(6), 1008-1014.

Greve, K. (2005-2010). Die Farbe in der Computergrafik. Kapitel 1.7. from http://farbe-computer.de/kapitel17.html

Gurram, M. M. (2013). Ophthalmic cell-phone imaging system: a costless imaging system. Can J Ophthalmol, 48(5), e135-139. doi: 10.1016/j.jcjo.2013.06.007 
Haddock, L. J., Kim, D. Y., \& Mukai, S. (2013). Simple, inexpensive technique for high-quality smartphone fundus photography in human and animal eyes. $J$ Ophthalmol, 2013, 518479. doi: 10.1155/2013/518479

Heard, P. F., Stone, C. J., Gregory, R. L., \& Marmion, V. J. (1987). A new computer graphics test for red/green colour anomaly,. In G. Verriest (Ed.), Colour Vision Deficiencies VIII (Vol. 46, pp. 181-194). Dodrecht: Martinus Nijhoff/Dr. W. Junk.

Heinsius, E. (1973). Die Farbsinnstörungen und ihre Prüfung in der Praxis. Stuttgart: Enke Verlag.

Helmholtz, v. H. (1867). Handbuch der physiologischen Optik. Leipzig: Leopold Voss.

Hering, E. (1874). Zur Lehre vom Lichtsinn. Wien: Akad Wiss, MathematNaturwiss.

Hoffmann, A., \& Menozzi, M. (1998). Computer-basierte Bestimmung von Rot/Grün-Farbanomalien. Biomed Tech (Berl), 43(5), 124-132.

Hong, J. I., \& Chang, B. Y. (2014). Development of the smartphone-based colorimetry for multi-analyte sensing arrays. Lab Chip, 14(10), 1725-1732. doi: 10.1039/c3lc51451j

Hovis, J. K., Cawker, C. L., \& Cranton, D. (1996). Comparison of the standard pseudoisochromatic plates--Parts 1 and 2--As screening tests for congenital red-green color vision deficiencies. J Am Optom Assoc, 67(6), 320-326.

Hyon, J. Y., Lee, J. H., \& Wee, W. R. (2005). Shift of colorimetric values in ishihara pseudoisochromatic plates with plate aging. Korean $\mathrm{J}$ Ophthalmol, 19(2), 145-148.

Ing, E. B., Parker, J. A., \& Emerton, L. A. (1994). Computerized colour vision testing. Can J Ophthalmol, 29(3), 125-128.

iPad. (2015a). iPad Modelle vergleichen. from http://www.apple.com/de/ipad/compare/

iPad. (2015b). Technische Daten-iPad. from https://support.apple.com/de_DE/specs/ipad

Iregren, A., Andersson, M., \& Nylen, P. (2002). Color vision and occupational chemical exposures: I. An overview of tests and effects. Neurotoxicology, 23(6), 719-733. doi: 10.1016/S0161-813X(02)00088-8

Jacobs, G. H. (1976). Color vision. Annu Rev Psychol, 27, 63-89. doi: 10.1146/annurev.ps.27.020176.000431

Jaeger, W. (1954). [Hereditary optic atrophy with dominant transmission; with special reference to the associated color-sense disorder]. Albrecht Von Graefes Arch Ophthalmol, 155(5), 457-484.

Jaeger, W. (1977). [Acquired colour-vision-deficiencies caused by side-effects of pharmacotherapy (author's transl)]. Klin Monbl Augenheilkd, 170(3), 453460.

Jaeger, W., \& Schneider, V. J. (1976). Colour vision deficiencies and haemophilia. Mod Probl Ophthalmol, 17, 143-146. 
Jagle, H., et al. (2004). Visual short-term effects of Viagra: double-blind study in healthy young subjects. Am J Ophthalmol, 137(5), 842-849. doi:

10.1016/j.ajo.2003.11.081

Kalmus, H. (1955a). The familial distribution of congenital tritanopia with some remarks on similar conditions. Ann Hum Genet, 20, 39-56.

Kalmus, H. (1955b). The familial distribution of congenital tritanopia, with some remarks on some similar conditions. Ann Hum Genet, 20(1), 39-56.

Kaluiny, J., Markowski, D., Kaluzny, B. J., \& Sikorski, B. (2006). [Did Leon Wyczokowski suffer from solar maculopathy?]. Klin Oczna, 108(10-12), 489-492.

Kinnear, P. R., Aspinall, P. A., \& Lakowski, R. (1972). The diabetic eye and colour vision. Trans Ophthalmol Soc U K, 92, 69-78.

Kolb, H. (1995a). Circuitry for Rod Signals through the Retina. In H. Kolb, E. Fernandez \& R. Nelson (Eds.), Webvision: The Organization of the Retina and Visual System. Salt Lake City (UT).

Kolb, H. (1995b). Cone Pathways through the Retina. In H. Kolb, E. Fernandez \& R. Nelson (Eds.), Webvision: The Organization of the Retina and Visual System. Salt Lake City (UT).

Kolb, H. (1995c). Midget pathways of the primate retina underlie resolution and red green color opponency. In H. Kolb, E. Fernandez \& R. Nelson (Eds.), Webvision: The Organization of the Retina and Visual System. Salt Lake City (UT).

Kolb, H. (1995d). Neurotransmitters in the Retina. In H. Kolb, E. Fernandez \& R. Nelson (Eds.), Webvision: The Organization of the Retina and Visual System. Salt Lake City (UT).

Kolb, H. (1995e). Photoreceptors. In H. Kolb, E. Fernandez \& R. Nelson (Eds.), Webvision: The Organization of the Retina and Visual System. Salt Lake City (UT).

Kolb, H. (1995f). Roles of Amacrine Cells. In H. Kolb, E. Fernandez \& R. Nelson (Eds.), Webvision: The Organization of the Retina and Visual System. Salt Lake City (UT).

Kolb, H. (1995g). S-Cone Pathways. In H. Kolb, E. Fernandez \& R. Nelson (Eds.), Webvision: The Organization of the Retina and Visual System. Salt Lake City (UT).

Kolb, H. (1995h). Simple Anatomy of the Retina. In H. Kolb, E. Fernandez \& R. Nelson (Eds.), Webvision: The Organization of the Retina and Visual System. Salt Lake City (UT).

Kolb, H., \& Nelson, R. (1996). Hyperpolarizing, small-field, amacrine cells in cone pathways of cat retina. J Comp Neurol, 371(3), 415-436. doi: 10.1002/(SICI)1096-9861(19960729)371:3\&lt;415::AIDCNE5\&gt;3.0.CO;2-5

Köllner, H. (1912). Die Störungen des Farbensinnes. Berlin: Karger Verlag.

Koshy, E., Car, J., \& Majeed, A. (2008). Effectiveness of mobile-phone short message service (SMS) reminders for ophthalmology outpatient appointments: observational study. BMC Ophthalmol, 8, 9. doi: 10.1186/1471-2415-8-9 
Kozak, L. C., \& Bullimore, M. A. (1994). Visual changes in human immunodeficiency virus infection. Optom Vis Sci, 71(9), 557-561.

Krastel, H. (1995). Farbsinn. In W. Straub, P. Kroll \& H. J. Küchle (Eds.), Augenärztliche Untersuchungsmethoden

(2., völlig neubearb. Auflage ed., pp. 537-566). Stuttgart: Enke Verlag.

Krastel, H. (2007). Farbensinnprüfung in der Praxis. Klin Monatsbl Augenheilkd, 224, 29-56.

Krastel, H., Kolling, G., Schiefer, U., \& Bach, M. (2009). [Quality demands on the assessment of colour vision]. Ophthalmologe, 106(12), 1083-1102. doi: 10.1007/s00347-009-1976-3

Kuchenbecker, J., Nicklas, S., \& Behrens-Baumann, W. (2010). Colourimetric analysis of three editions of the Velhagen-Broschmann pseudoisochromatic colour plates. Ophthalmologica, 224, 47-54

Kuchenbecker, J., Roehl, F., Bernarding, J., \& Behrens-Baumann, W. (2010). [Test-retest reliability of a web-based colour vision test]. Klin Monbl Augenheilkd, 227(11), 892-896. doi: 10.1055/s-0029-1245314

Kuchenbecker, J., Röhl, F. W., Weßelburg, A., Bernarding, J., \& BehrensBaumann, W. (2007). Untersuchungen zur Validität eines web-basierten Farbsehtest für Screeninguntersuchungen des Farbensehens. Ophthalmologe, 104(1), 47-53.

Kumar, S., Wang, E. H., Pokabla, M. J., \& Noecker, R. J. (2012). Teleophthalmology assessment of diabetic retinopathy fundus images: smartphone versus standard office computer workstation. Telemed J E Health, 18(2), 158-162. doi: 10.1089/tmj.2011.0089

Kurtenbach, A., Flogel, W., \& Erb, C. (2002). Anomaloscope matches in patients with diabetes mellitus. Graefes Arch Clin Exp Ophthalmol, 240(2), 79-84.

Kurtenbach, A., et al. (1994). Brightness matching and colour discrimination in young diabetics without retinopathy. Vision Res, 34(1), 115-122.

Lachenmayr B., F. D., Hartmann E., Buser A. (2006). Augen-Brille-Refraktion. Stuttgart.

Lamirel, C., Bruce, B. B., Wright, D. W., Newman, N. J., \& Biousse, V. (2012). Nonmydriatic digital ocular fundus photography on the iPhone 3G: the FOTO-ED study. Arch Ophthalmol, 130(7), 939-940. doi:

10.1001/archophthalmol.2011.2488

Landis, J. R., \& Koch, G. G. (1977). The measurement of observer agreement for categorical data. Biometrics, 33(1), 159-174.

Lang, G. K. (2000). Augenheilkunde, Verstehen-Lernen-Anwenden, Histologie und Funktion der Netzhautschichten (2. Auflage ed.). Stuttgart: Georg Thieme Verlag.

Lanthony, P. (1986). [The Tritan album: a pseudo-isochromatic album for the diagnosis of blue-yellow dyschromatopsias]. Bull Soc Ophtalmol Fr, 86(10), 1127-1132.

Lanthony, P. (1987). [Color vision]. Annee Ther Clin Ophtalmol, 38, 117-133.

Lawrenson, J. G., Kelly, C., Lawrenson, A. L., \& Birch, J. (2002). Acquired colour vision deficiency in patients receiving digoxin maintenance therapy. $\mathrm{Br} \mathrm{J}$ Ophthalmol, 86(11), 1259-1261. 
Lee, R. M., Oleszczuk, J. D., Hyer, J. N., \& Gazzard, G. (2014). Patient acceptance to smartphone technology to monitor and improve glaucoma health-care outcomes. Eye (Lond), 28(4), 496. doi: 10.1038/eye.2014.22

Lennie, P., \& D'Zmura, M. (1988). Mechanisms of color vision. Crit Rev Neurobiol, 3(4), 333-400.

Li, J., Tripathi, R. C., \& Tripathi, B. J. (2008). Drug-induced ocular disorders. Drug Saf, 31(2), 127-141.

Lord, K., Shah, V. A., \& Krishna, R. (2013). The Eye Handbook: a mobile app in ophthalmic medicine. Mo Med, 110(1), 49-51.

Luthke, S., Welters, A., Kropf, S., \& Bockelmann, I. (2010). [Color vision testing in patients with diabetes mellitus and arterial hypertension]. Ophthalmologe, 107(10), 923-929. doi: 10.1007/s00347-010-2169-9

M.Wellendorf. (2012). Das neue iPad im Test - Teil 2: Display- und Kameraqualität, Wärmentwicklung und Akku-Laufzeiten. from http://www.tomshardware.de/ipad-3-benchmark-retina-display,testberichte-2410442.html

Maamari, R. N., D'Ambrosio, M. V., Joseph, J. M., \& Tao, J. P. (2014). The efficacy of a novel mobile phone application for goldmann ptosis visual field interpretation. Ophthal Plast Reconstr Surg, 30(2), 141-145. doi: 10.1097/IOP.0000000000000030

Maamari, R. N., Keenan, J. D., Fletcher, D. A., \& Margolis, T. P. (2014). A mobile phone-based retinal camera for portable wide field imaging. $\mathrm{Br} \mathrm{J}$ Ophthalmol, 98(4), 438-441. doi: 10.1136/bjophthalmol-2013-303797

Maaranen, T. H., Tuppurainen, K. T., \& Mantyjarvi, M. I. (2000). Color vision defects after central serous chorioretinopathy. Retina, 20(6), 633-637.

Mantyjarvi, M., \& Terasvirta, M. (1992). Observations on color vision testing in ocular hypertension and glaucoma. Int Ophthalmol, 16(6), 417-422.

Mantyjarvi, M., \& Tuppurainen, K. (1992). Color vision in Stargardt's disease. Int Ophthalmol, 16(6), 423-428.

Mantyjarvi, M. I., Nerdrum, K., \& Tuppurainen, K. (1992). Color vision in dominant optic atrophy. J Clin Neuroophthalmol, 12(2), 98-103.

Marré, M., \& Marré, E. (1986). Erworbene Störungen des Farbensehen. Leipzig: Thieme Verlag.

Meena, M., \& Bhatia, K. (2013). Smart phone as an adjunctive imaging tool to visualize scolex in orbital myocysticercosis. Int Ophthalmol, 33(3), 319321. doi: 10.1007/s10792-012-9666-6

Merbs, S. L., \& Nathans, J. (1992). Absorption spectra of human cone pigments. Nature, 356(6368), 433-435. doi: 10.1038/356433a0

Michael, C. R. (1973). Color vision. N Engl J Med, 288(14), 724-728.

Mollon, J. D., \& Reffin, J. E. (1989). A computer-controlled colour vision test that combines the principles of Chibret and Stilling. J Physiol, 414, 5.

Motulsky, A. G. (1988). Normal and abnormal color-vision genes. Am J Hum Genet, 42(3), 405-407.

Murali, V. N., \& Coughlan, J. M. (2013). Smartphone-Based Crosswalk Detection and Localization for Visually Impaired Pedestrians. IEEE Int Conf Multimed Expo Workshops, 2013, 1-7. doi: 10.1109/ICMEW.2013.6618432 
Nakajima, A., Ichikawa, H., Nakagawa, O., Majima, A., \& Watanabe, M. (1960). Ishihara test in color-vision defects. Studies on a statistical method for evaluation of the screening efficiency of several plates. Am J Ophthalmol, 49, 921-929.

Nathans, J. (1989). The genes for color vision. Sci Am, 260(2), 42-49.

Nathans, J. (1999). The evolution and physiology of human color vision: insights from molecular genetic studies of visual pigments. Neuron, 24(2), 299312.

Nathans, J., et al. (1989). Molecular genetics of human blue cone monochromacy. Science, 245(4920), 831-838.

Nathans, J., Merbs, S. L., Sung, C. H., Weitz, C. J., \& Wang, Y. (1992). Molecular genetics of human visual pigments. Annu Rev Genet, 26, 403424. doi: 10.1146/annurev.ge.26.120192.002155

Nathans, J., Piantanida, T. P., Eddy, R. L., Shows, T. B., \& Hogness, D. S. (1986). Molecular genetics of inherited variation in human color vision. Science, 232(4747), 203-210.

Nathans, J., et al. (1992). Visual pigments and inherited variation in human vision. Soc Gen Physiol Ser, 47, 109-131.

Nathans, J., Thomas, D., \& Hogness, D. S. (1986). Molecular genetics of human color vision: the genes encoding blue, green, and red pigments. Science, 232(4747), 193-202.

Neitz, M., \& Neitz, J. (2000). Molecular genetics of color vision and color vision defects. Arch Ophthalmol, 118(5), 691-700.

Oculus. HMC Anomaloskop MR-HMC Anomaloskop R-Gebrauchsanweisung.

Osterberg, G. (1935). Topography of the layer of rods and cones in the human retina. Acta Ophthal supp., 6:1-103.

Pearlman, A. L., Birch, J., \& Meadows, J. C. (1978). Cerebral color blindness: an acquired defect in hue discrimination. Trans Am Neurol Assoc, 103, 133134.

Pease, P. L. (1998). Color vision In: Benjamin, W.J.(Ed), Borishis Clinical Refraction.Philadelphia:W.B. Saunders Company, Chapter 9.

Pinckers, A., Nabbe, B., \& Vossen, H. (1985). Standard Pseudoisochromatic Plates part 2. Ophthalmologica, 190(2), 118-124.

Rand, G., \& Rittler, M. C. (1956). An evaluation of the AO H-R-R pseudoisochromatic plates; a test for detecting, classifying, and estimating the degree of defective color vision. AMA Arch Ophthalmol, 56(5), 736742.

Reffin, J. P., Astell, S., \& Mollon, J. D. (1991). Trials of a computer-controlled colour vision test that preserves the advantages of pseudoisochromatic plates. In B. Drum, J. D. Moreland \& A. Serra (Eds.), Colour Vision Deficiencies $X$ (pp. 69-76). Dordrecht: Kluwer Academic Publishers.

Ripps, H., \& Weale, R. A. (1969). Color vision. Annu Rev Psychol, 20, 193-216. doi: 10.1146/annurev.ps.20.020169.001205

Rizzo, M., Smith, V., Pokorny, J., \& Damasio, A. R. (1993). Color perception profiles in central achromatopsia. Neurology, 43(5), 995-1001. 
Röhl, F.-W., Theuerkauf, A., Kuchenbecker, J., \& Weßelburg, A. (2007). Einsatz eines web-basierten Farbsehtest für Screeninguntersuchungen des Farbensehens. Paper presented at the GMDS-Tagung Augsburg.

Roorda, A., \& Williams, D. R. (1999). The arrangement of the three cone classes in the living human eye. Nature, 397(6719), 520-522. doi: 10.1038/17383

Roy, M. S., Gunkel, R. D., \& Podgor, M. J. (1986). Color vision defects in early diabetic retinopathy. Arch Ophthalmol, 104(2), 225-228.

Roy, M. S., Rodgers, G., Gunkel, R., Noguchi, C., \& Schechter, A. (1987). Color vision defects in sickle cell anemia. Arch Ophthalmol, 105(12), 1676-1678.

Ruamviboonsuk, P., Sudsakorn, N., Somkijrungroj, T., Engkagul, C., \& Tiensuwan, M. (2012). Reliability of visual acuity measurements taken with a notebook and a tablet computer in participants who were illiterate to Roman characters. J Med Assoc Thai, 95 Supp/ 3, S109-116.

Russo, A., Morescalchi, F., Costagliola, C., Delcassi, L., \& Semeraro, F. (2015). Comparison of smartphone ophthalmoscopy with slit-lamp biomicroscopy for grading diabetic retinopathy. Am J Ophthalmol, 159(2), 360-364 e361. doi: 10.1016/j.ajo.2014.11.008

Shanmugam, P. M., Mishra, D., \& Ramanjulu, R. (2014). Fluorescein fundus angiography using a smartphone. Retina, 34(3), e6-7. doi: 10.1097/IAE.0000000000000132

Sharpe, L. T., et al. (1998). Red, green, and red-green hybrid pigments in the human retina: correlations between deduced protein sequences and psychophysically measured spectral sensitivities. J Neurosci, 18(23), 10053-10069.

Sommerhalder, J., et al. (1998). Colour vision in AIDS patients without HIV retinopathy. Vision Res, 38(21), 3441-3446.

Spektrum. (2015). Anomaloskop: Prüffeld zum Ermitteln von Farbsinnstörungen., from http://www.spektrum.de/lexikon/physik/anomaloskop/567

Spektrum.de. (2015). Farbverwechselung from http://www.spektrum.de/lexikon/optik/farbverwechslung/931

Stanzel, B. V., \& Meyer, C. H. (2012). [Smartphones in ophthalmology : Relief or toys for physicians?]. Ophthalmologe, 109(1), 8-20. doi: 10.1007/s00347011-2425-7

Stilling, J. (1877). Die Prüfung des Farbsinns beim Eisenbahn- und Marinepersonal. Cassel: Fischer.

Suto, S. e. a. (2014a). Fluorescein fundus angiography with smartphone. Retina, 34(1), 203-205. doi: 10.1097/IAE.0000000000000041

Suto, S. e. a. (2014b). [Photography of anterior eye segment and fundus with smartphone]. Nihon Ganka Gakkai Zasshi, 118(1), 7-14.

Swanson, W. H., \& Cohen, J. M. (2003). Color vision. Ophthalmol Clin North Am, 16(2), 179-203.

Tablet-PCs.eu. (2015a). iPad - Das Trend-Tablet von Apple.

Tablet-PCs.eu. (2015b). Tablet-Funktionen. from http://www.tablet-pcs.eu/tabletfunktionen.html 
Thompson, D. G., Howarth, F., Taylor, H., Levy, I. S., \& Birch, J. (1979). Defective colour vision in diabetes: a hazard to management. Br Med $\mathrm{J}$, 1(6167), 859-860.

Tietjen, A., et al. (2013). [New options for digital photo documentation during routine examination for ophthalmologists]. Klin Monbl Augenheilkd, 230(6), 604-610. doi: 10.1055/s-0032-1328407

Trusov, M. S. (1972). [Color vision disorders in hypertonic disease and arteriosclerosis]. Oftalmol Zh, 27(1), 19-22.

Velhagen, K., \& Broschmann, D. (1995). Tafeln zur Prüfung des Farbensinnes (30., unveränd. Auflage ed.). Stuttgart New-York: Thieme Verlag.

Velhagen, K., \& Broschmann, D. (1997). Tafeln zur Prüfung des Farbensinnes (31., unveränd. Auflage ed.). Stuttgart New-York: Thieme Verlag.

Velhagen, K., \& Broschmann, D. (2000). Tafeln zur Prüfung des Farbensinnes (32., unveränd. Auflage ed.). Stuttgart New-York: Thieme Verlag.

Verriest, G. (1963). Further studies on aquired deficiency of color discrimination. J Opt Soc Amer, 53, 185-195.

Vistamehr, S., Walsh, T. J., \& Adelman, R. A. (2007). Ethambutol neuroretinopathy. Semin Ophthalmol, 22(3), 141-146. doi: 10.1080/08820530701457134

Vola, J. L., Riss, M., Jayle, G. E., Gosset, A., \& Tassy, A. (1972). Acquired deficiency of colour vision in lateral homonymous hemianopsia. Mod Probl Ophthalmol, 11, 150-159.

Walraven, P. L. (1972). Color vision. Annu Rev Psychol, 23, 347-374. doi: 10.1146/annurev.ps.23.020172.002023

Weitz, C. J., et al. (1992). Human tritanopia associated with two amino acid substitutions in the blue-sensitive opsin. Am J Hum Genet, 50(3), 498-507.

Weitz, C. J., Went, L. N., \& Nathans, J. (1992). Human tritanopia associated with a third amino acid substitution in the blue-sensitive visual pigment. $A m \mathrm{~J}$ Hum Genet, 51(2), 444-446.

Wikipedia. (2010). CIE-Normvalenzsystem. from http://de.wikipedia.org/wiki/CIENormvalenzsystem

Wikipedia. (2015a). Android. from http://de.wikipedia.org/wiki/Android (Betriebssystem)

Wikipedia. (2015b). Farbraum. from http://de.wikipedia.org/wiki/Farbraum

Wikipedia. (2015c). Retina Display. from http://de.wikipedia.org/wiki/Retina-Display

Wikipedia. (2015d). RGB-Farbraum. from http://de.wikipedia.org/wiki/RGB-Farbraum Adobe-RGB-.281998.29-Farbraum

Wikipedia. (2015e). Tabletcomputer. from http://de.wikipedia.org/wiki/Tabletcomputer

Wikipedia. (2015f). Touchscreen. from http://de.wikipedia.org/wiki/Touchscreen

Wilkipedia. (2015). Munsell-Farbsystem. from http://de.wikipedia.org/wiki/MunsellFarbsystem

Wright, W. (1952). The characteristics o tritanopia. J Opt Soc Amer, 42:509-520.

Yamaguchi, T., Motulsky, A. G., \& Deeb, S. S. (1997). Visual pigment gene structure and expression in human retinae. Hum Mol Genet, 6(7), 981990.

Young, T. (1802). On the theory of light and colour. Phil Trans Roy Soc, 20-71. 
Zhang, Z. T., Zhang, S. C., Huang, X. G., \& Liang, L. Y. (2013). A pilot trial of the iPad tablet computer as a portable device for visual acuity testing. $J$ Telemed Telecare, 19(1), 55-59. doi: 10.1177/1357633X12474964

Zhigang Xiang, R. A. P. (2000). Computergrafik.

Zrenner, E. (1985). Farbsinnprüfungen: Grundlagen, Meßverfahren und Anwendungen bei angeborenen und erworbenen Farbsinnstörungen. In $\mathrm{O}$. E. Lund \& T. N. Waubke (Eds.), Augenerkrankungen im Kindesalter (Vol. 106, pp. 263-286).

Zrenner, E. (1987). Untersuchungsstrategien bei Farbsinnstörungen. In O. E. Lund \& T. N. Waubke (Eds.), Okuläre Symptome, Strategien der Untersuchung (Vol. 112, pp. 84-101). Stuttgart: Enke Verlag.

Zrenner, E., Riedel, K. G., Adamczyk, R., Gilg, T., \& Liebhardt, E. (1986). Effects of ethyl alcohol on the electrooculogram and color vision. Doc Ophthalmol, 63(4), 305-312.

Zvornicanin, E., Zvornicanin, J., \& Hadziefendic, B. (2014). The Use of Smart phones in Ophthalmology. Acta Inform Med, 22(3), 206-209. doi: 10.5455/aim.2014.22.206-209 


\section{Danksagung}

Eine wissenschaftliche Arbeit ist nie das Werk einer einzelnen Person, deshalb ist es jetzt an der Zeit, mich bei allen Menschen zu bedanken, die mir die Erstellung meiner Dissertation ermöglicht haben.

An der ersten Stelle gilt mein Dank Herr Privat Dozent Dr. med. J. Kuchenbecker für die Idee zu diesem interessanten Promotionsthema und die exzellente Betreuung. Seine motivierende Unterstützung und Hilfsbereitschaft bei allen Fragen und Problemen haben entscheidend zum Gelingen dieser Arbeit beigetragen. Ohne seinen wertvollen akademischen Rat wäre diese Arbeit nicht entstanden.

Weiterhin möchte ich mich ebenfalls bei Dr. U. Gauger für die Auswertung der Daten, insbesondere für die Beratung und die Auswahl der geeigneten statistischen Testverfahren bedanken.

Mein Dankeschön gilt ebenfalls allen Probanden für ihre freiwillige Bereitschaft an den Untersuchungen teilzunehmen.

Ein herzliches Dankeschön möchte ich meinen Eltern aussprechen, die diese Arbeit in allen Phasen mit jeder möglichen Unterstützung bedacht haben.

Mein besonderer Dank gilt meinem Mann, Krisos, ohne seine liebevolle Fürsorge wäre diese Arbeit nicht zu dem Werk geworden, das es heute ist. 


\section{Ehrenerklärung}

Ich erkläre, dass ich die der Medizinischen Fakultät der Otto-von-GuerickeUniversität zur Promotion eingereichte Dissertation mit dem Titel Untersuchung des Farbsehens mittels Pigmentfarbtafeln und Tablet-PC (iPad $2 \AA)$

mit Unterstützung durch Herrn Privat Dozent Dr. med. J. Kuchenbecker und den Statistiker Herrn Dr. U. Gauger ohne sonstige Hilfe durchgeführt und bei der Abfassung der Dissertation keine anderen als die dort aufgeführten Hilfsmittel benutzt habe.

Bei der Abfassung der Dissertation sind Rechte Dritter nicht verletzt worden.

Ich habe diese Dissertation bisher an keiner in- oder ausländischen Hochschule zur Promotion eingereicht. Ich übertrage der Medizinischen Fakultät das Recht, weitere Kopien meiner Dissertation herzustellen und zu vertreiben.

Berlin, 15. Juli 2015

Panagiota Tsimpri 


\section{Lebenslauf}

\section{Personaldaten}

Name: Panagiota Tsimpri

Geburtstag/-ort: 11.12.1984, Athen (Griechenland)

Adresse: Barnimstraße 38, Berlin 10249

Staatsbürgerschaft: Griechisch

Telefonnummer: 015117282651

E-Mail: panagiotatsimpri@hotmail.com

Familienstand: verheiratet

\section{Beruflicher Werdegang und Facharztausbildung}

01.09.2014 bis heute: Fachärztin an der Klinik für Augenheilkunde Berlin Buch

11.08.2014: Fachärztin für Augenheilkunde

09.05.2014: Europäische Facharztprüfung und Erhalt des Titels FEBO (Fellow of the European Board of Opthalmology)

01.12.2011 bis 30.08.2014: Assistenzärztin an der Klinik für Augenheilkunde Berlin Buch

15.04.2010 bis 30.11.2011: Assistenzärztin an der Klinik für Augenheilkunde, St. Joseph-Stift Bremen

06.04.2009 bis 31.03.2010: Assistenzärztin an der Klinik für Augenheilkunde, SRH Zentralklinikum Suhl

\section{Approbation - Ärztekammer-Mitgliedschaft}

24.03.2009: Approbation als Ärztin in Deutschland

12.08.2008: Approbation als Ärztin in Griechenland 
22.06.2009: Mitgliedschaft in der Deutsche Ärztekammer

\section{Akademische Ausbildung}

30.09.2002 bis 21.07.2008: Medizinstudium an der Universität Patras

(Griechenland) - Fakultät für Gesundheitswissenschaften - Fachbereich Medizin

\section{Mitgliedschaften}

Berufsband der Augenärzte (BVA)

Deutsche Ophthalmologische Gesellschaft (DOG)

\section{Schulische Ausbildung}

1999-2002 : 1es Lyzeum Chaidari , Athene, Griechenland

07.2002 : Griechische Reifeprüfung

\section{Sprachen}

- Deutsch Niveau: Verhandlungssicher (C1)

- Englisch Niveau: Verhandlungssicher, Certificate of Proficiency in English (University of Cambridge)

- Griechisch Niveau: Muttersprache

\section{EDV-Kenntnisse}

Word, Excel, Internet

Berlin, 15. Juli 2015

Panagiota Tsimpri 


\section{Anhang 1}

Verwendete Pigmentfarbtafeln der 34. Auflage (2011)

(Velhagen/Broschmann/Kuchenbecker)

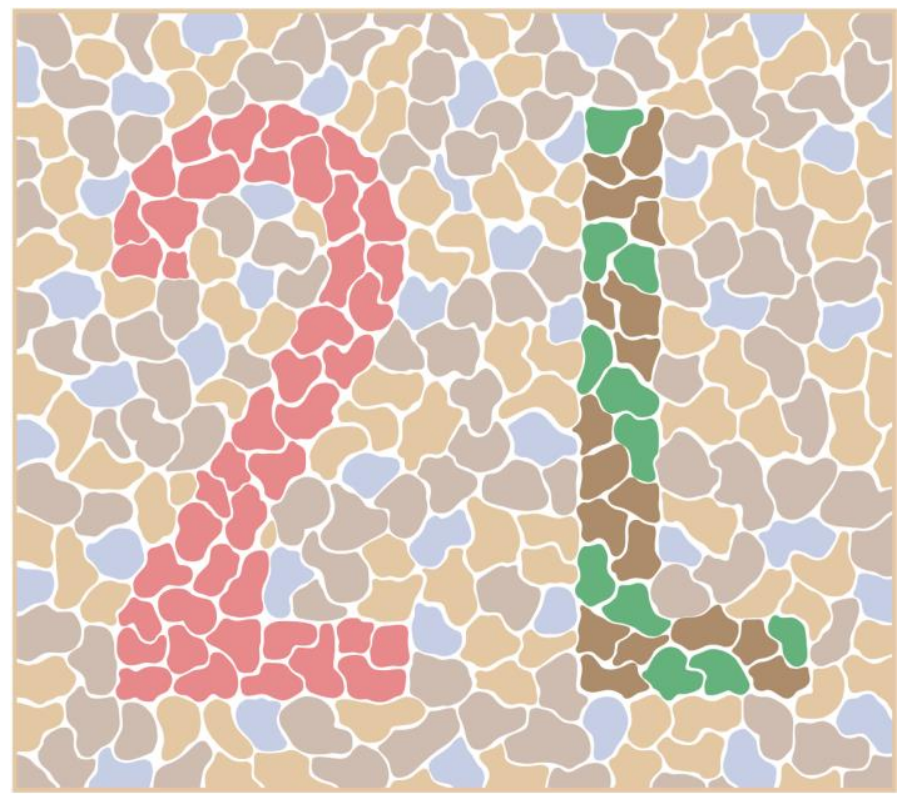

Tafel 1: Demonstrationstafel (Zeichen: 2L)

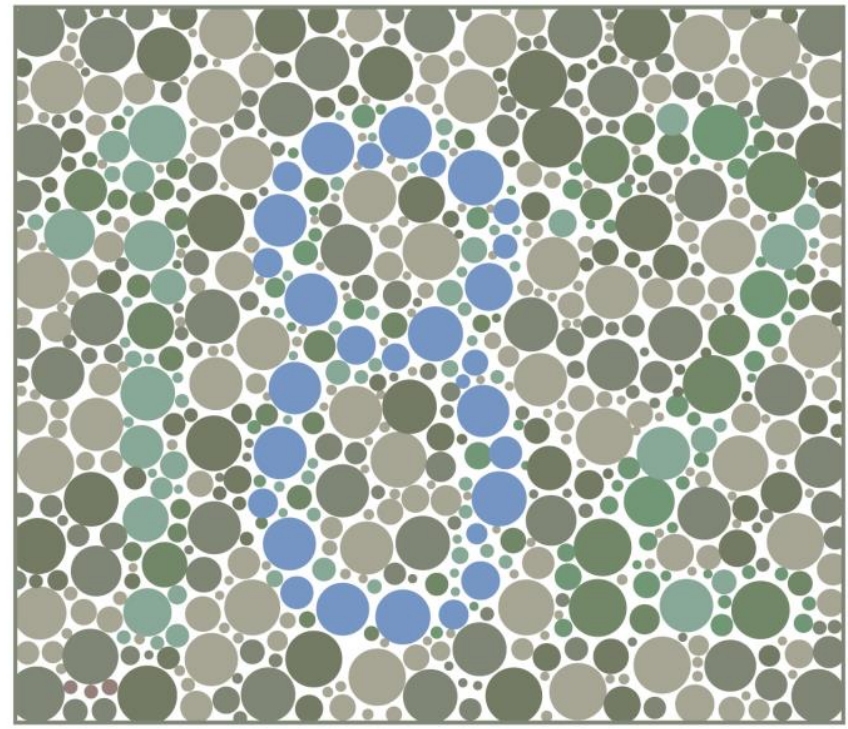

Tafel 2: Diagnostische Tafel (Zeichen: 182) 


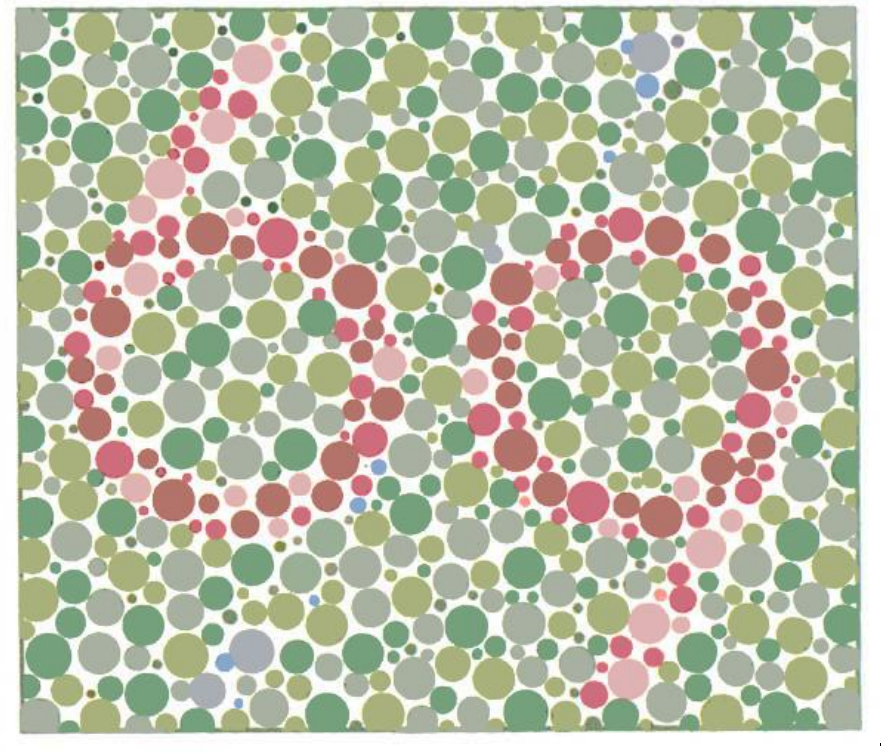

Tafel 3: Tafel mit alternativem Sehzeichen (Zeichen: 69)

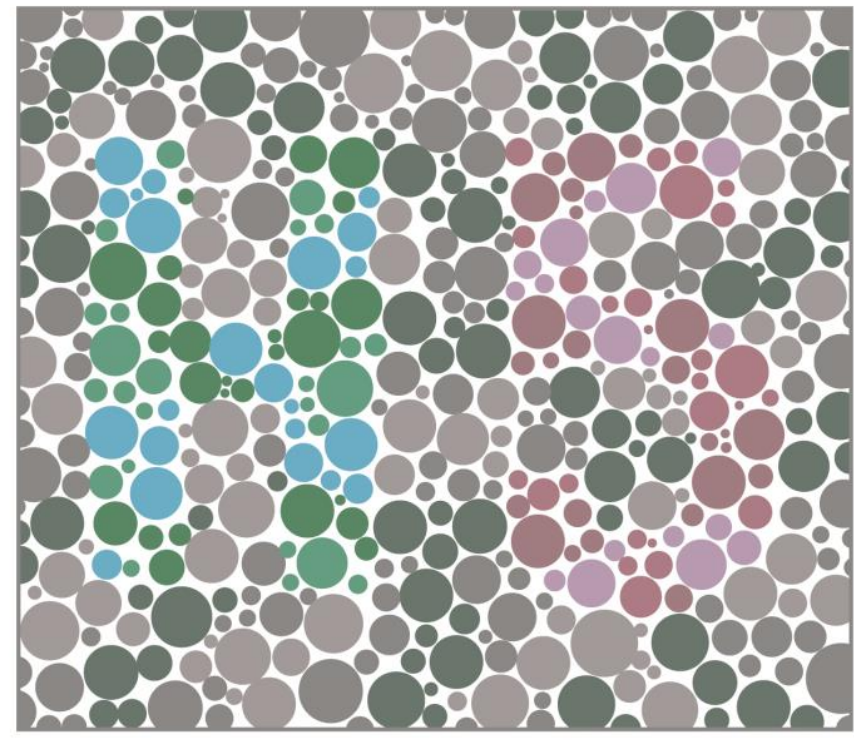

Tafel 4: Tafel mit verschwindenden Sehzeichen (Zeichen: H5) 


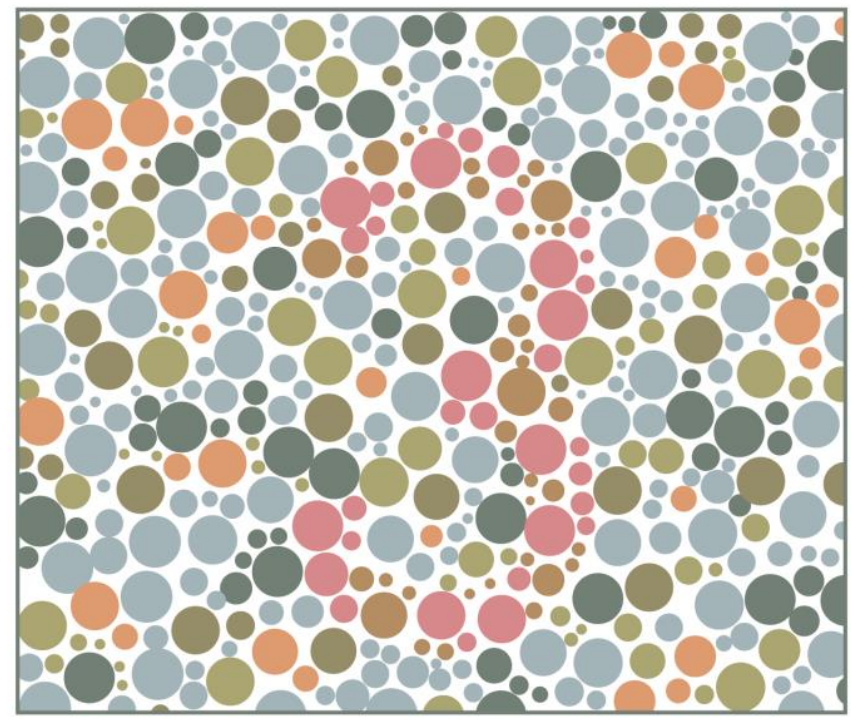

Tafel 5: Tafel mit verschwindenden Sehzeichen (Zeichen: 3)

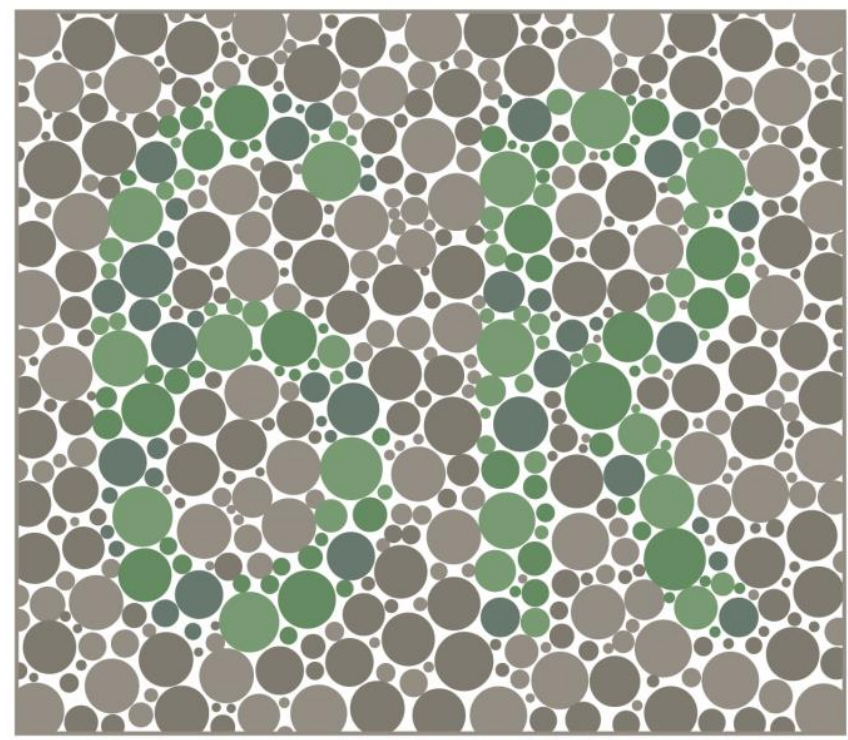

Tafel 6: Tafel mit verschwindenden Sehzeichen (Zeichen: 6R) 


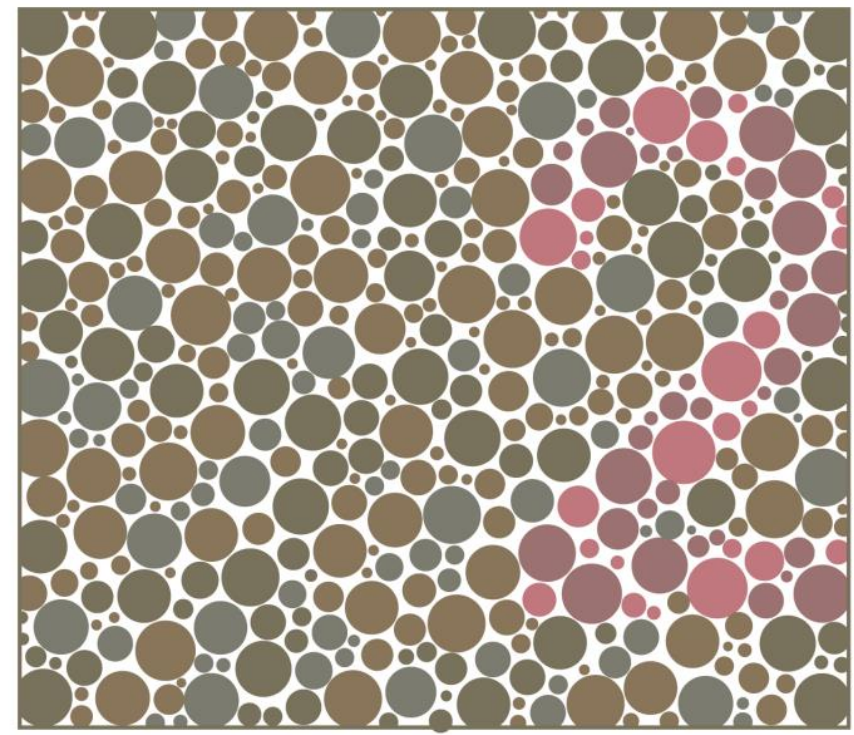

Tafel 7: Tafel mit verschwindenden Sehzeichen (Zeichen: 2)

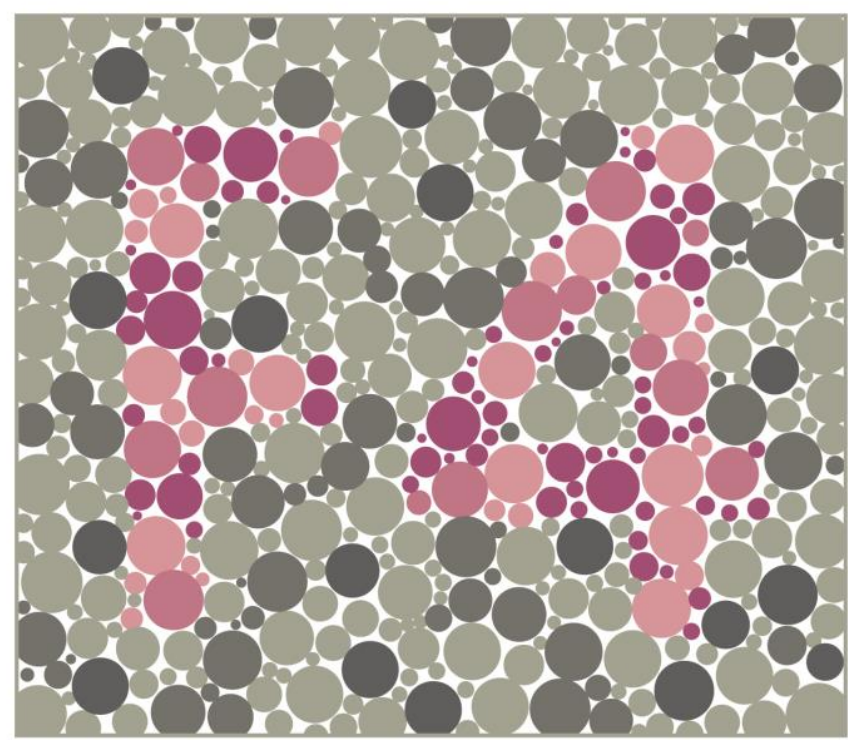

Tafel 8: Tafel mit verschwindenden Sehzeichen (Zeichen: F4) 


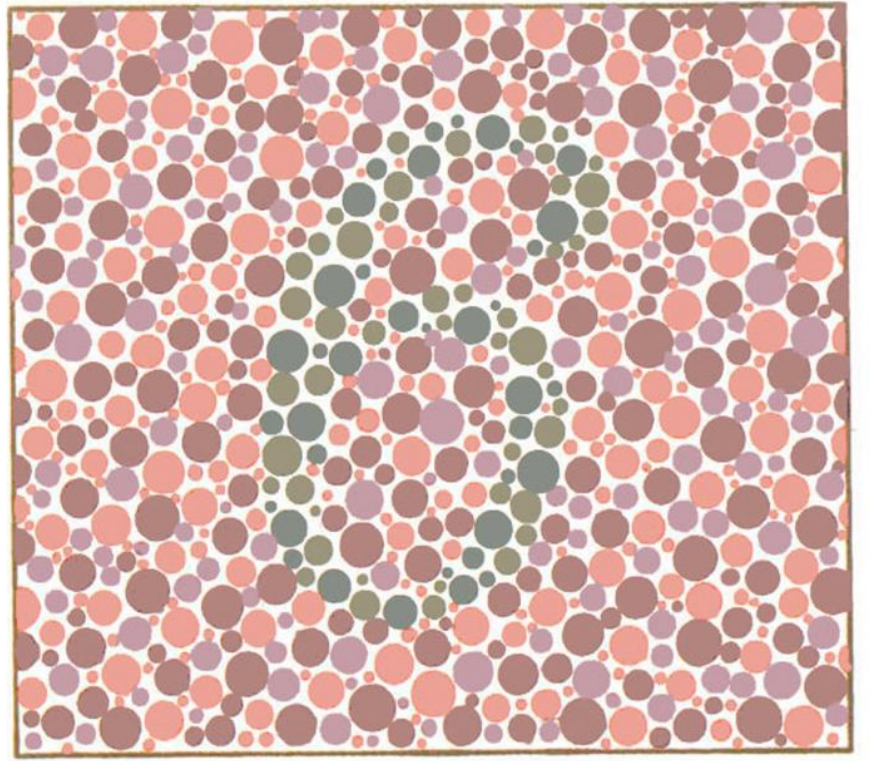

Tafel 9: Tafel mit verschwindenden Sehzeichen (Zeichen: 6)

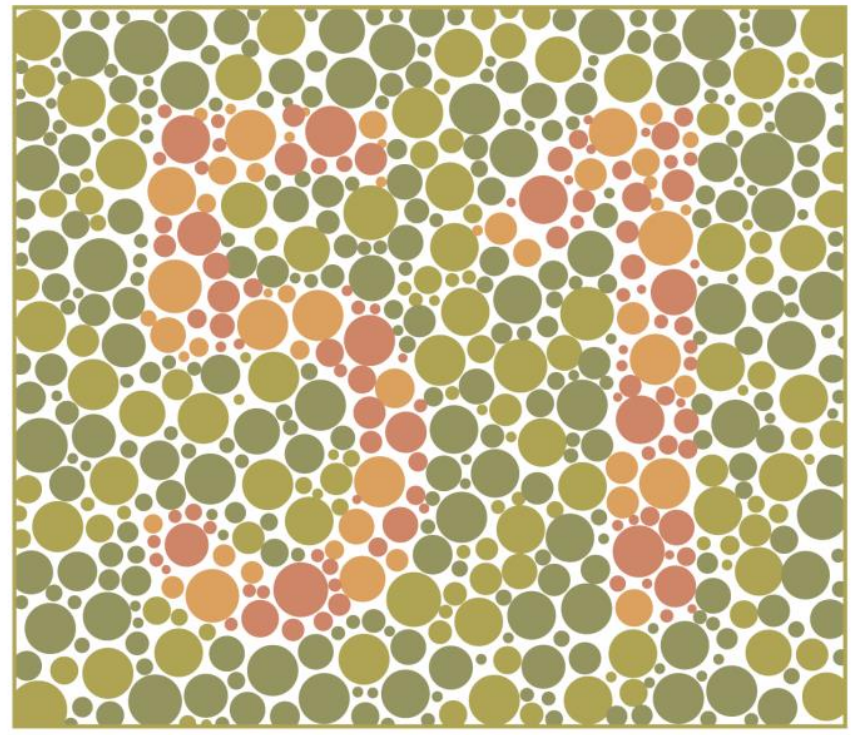

Tafel 10: Tafel mit verschwindenden Sehzeichen (Zeichen: 51) 


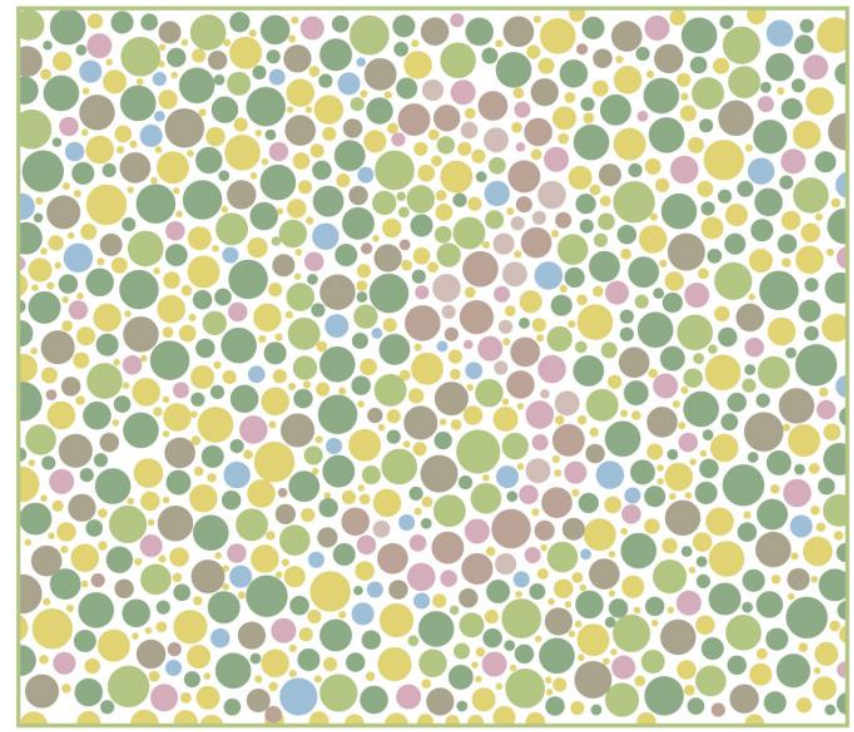

Tafel 11: Tafel mit alternativem Sehzeichen (Zeichen: 3)

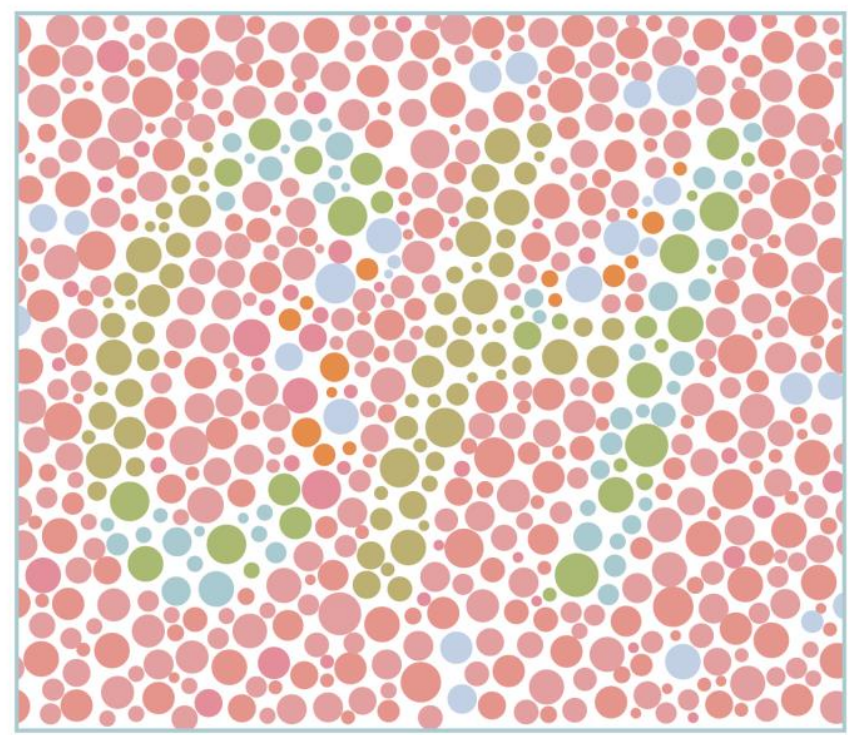

Tafel 12: Tafel mit alternativem Sehzeichen (Zeichen: $\mathrm{CH}$ ) 


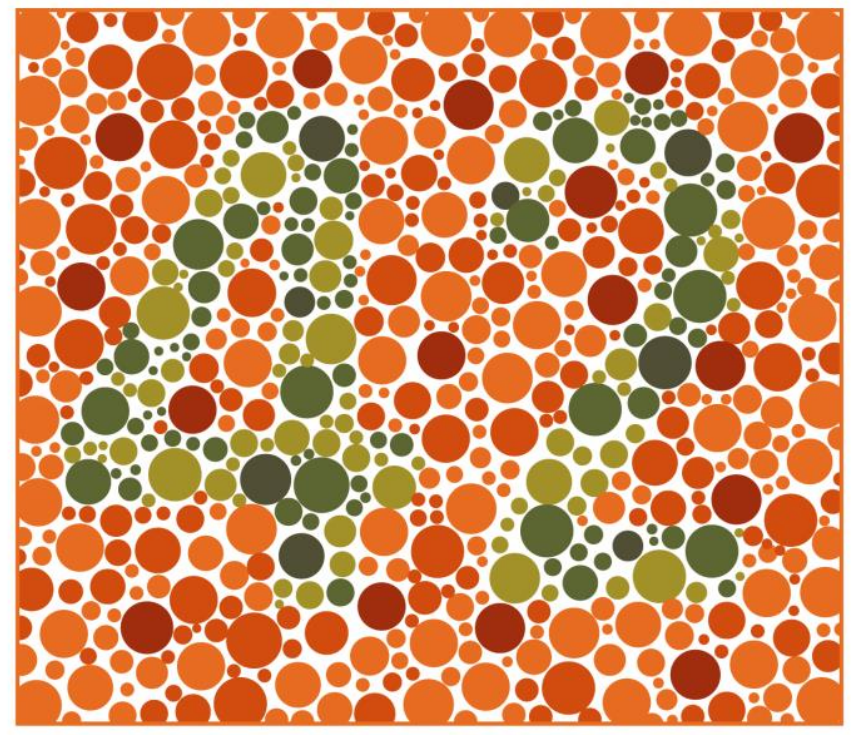

Tafel 13: Tafel mit verschwindenden Sehzeichen (Zeichen 42)

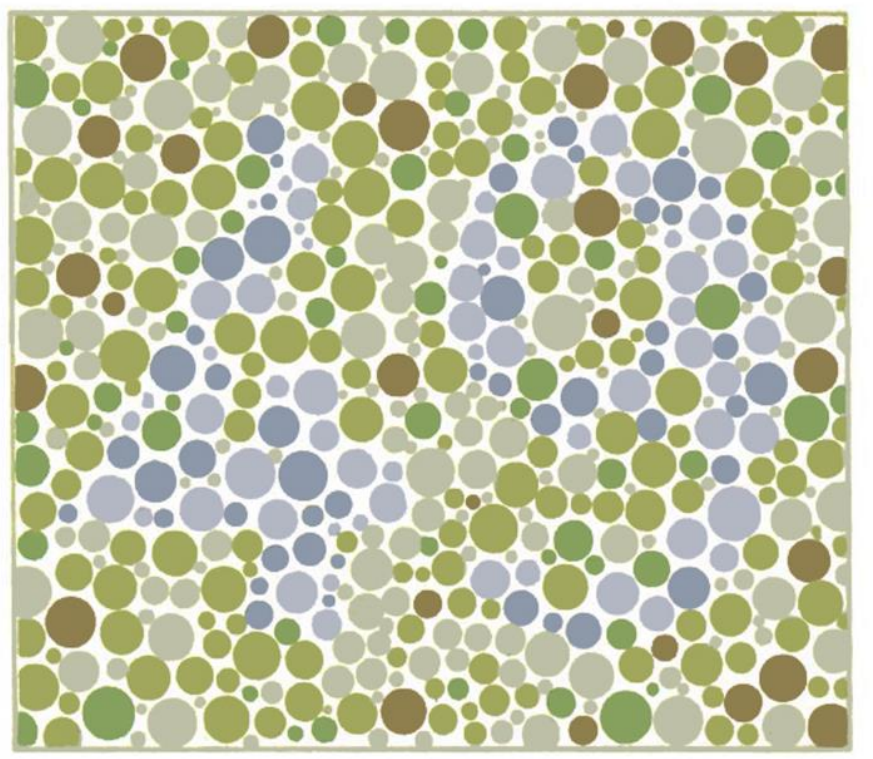

Tafel 14: Tafel mit verschwindenden Sehzeichen (Zeichen 49) 


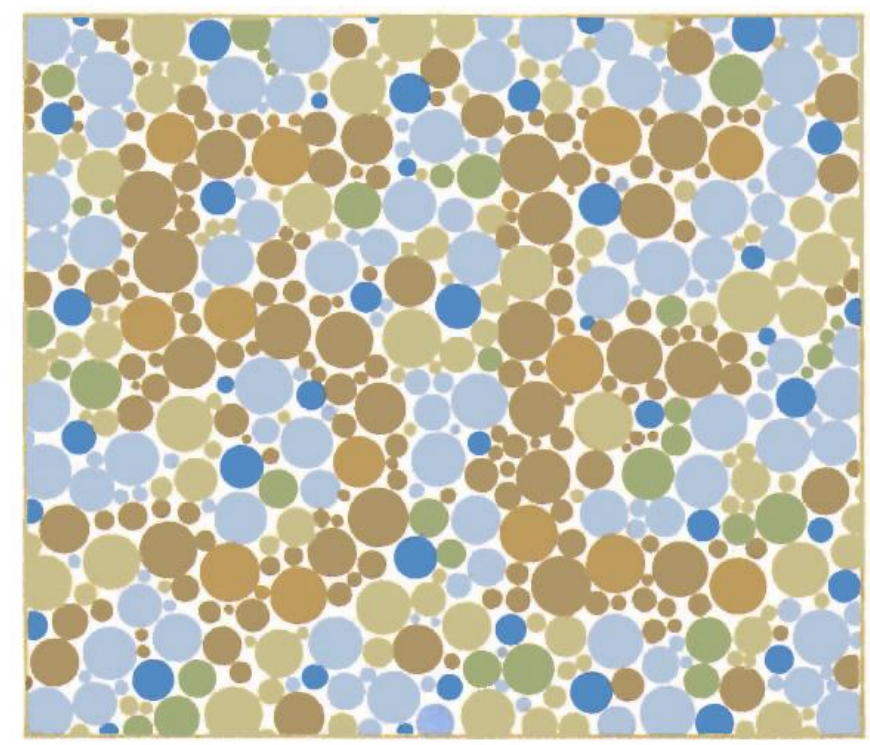

Tafel 15: Tafel mit verschwindenden Sehzeichen (Zeichen 5E)

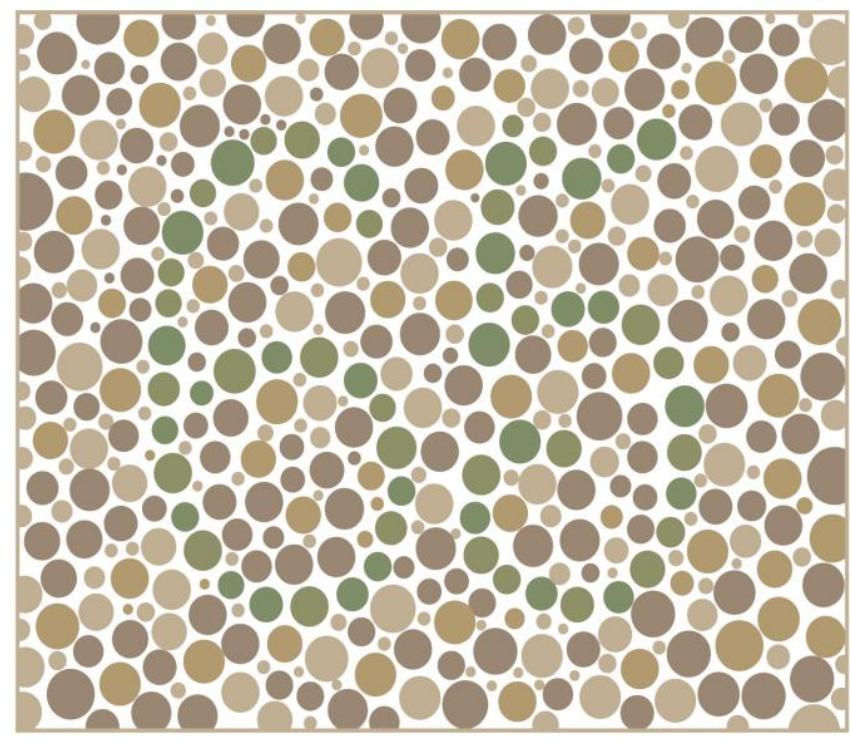

Tafel 16: Tafel mit verschwindenden Sehzeichen (Zeichen: 65) 


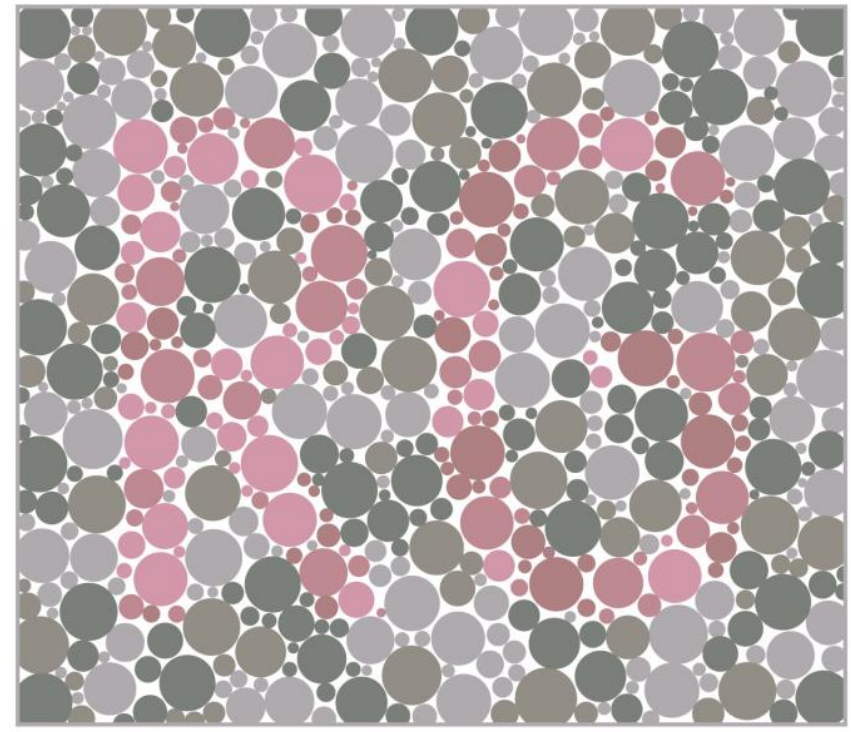

Tafel 17: Tafel mit verschwindenden Sehzeichen (Zeichen: RG)

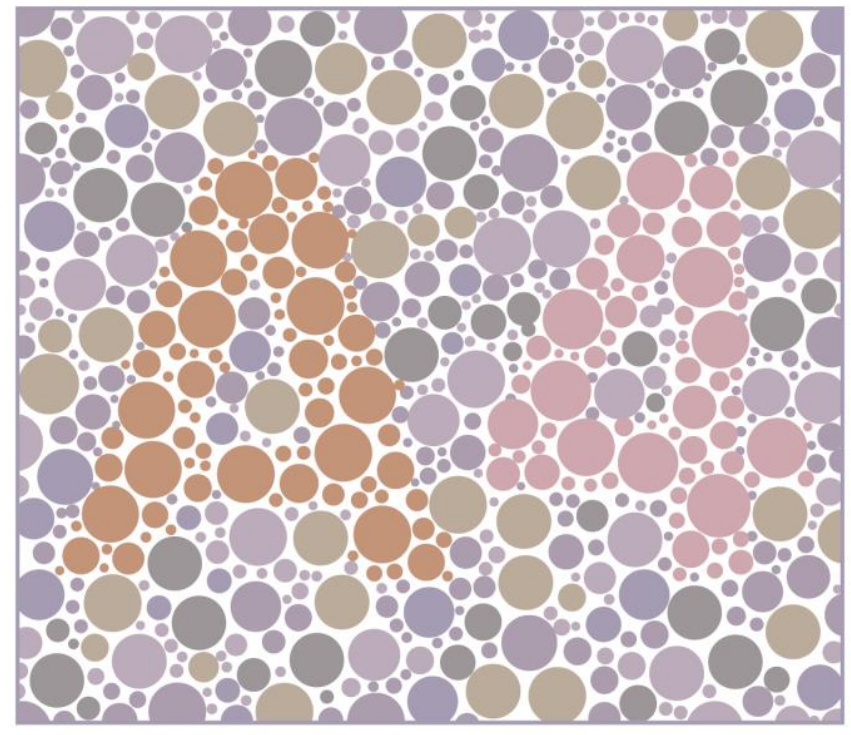

Tafel 18: Tafel mit verschwindenden Sehzeichen (Zeichen: A4) 


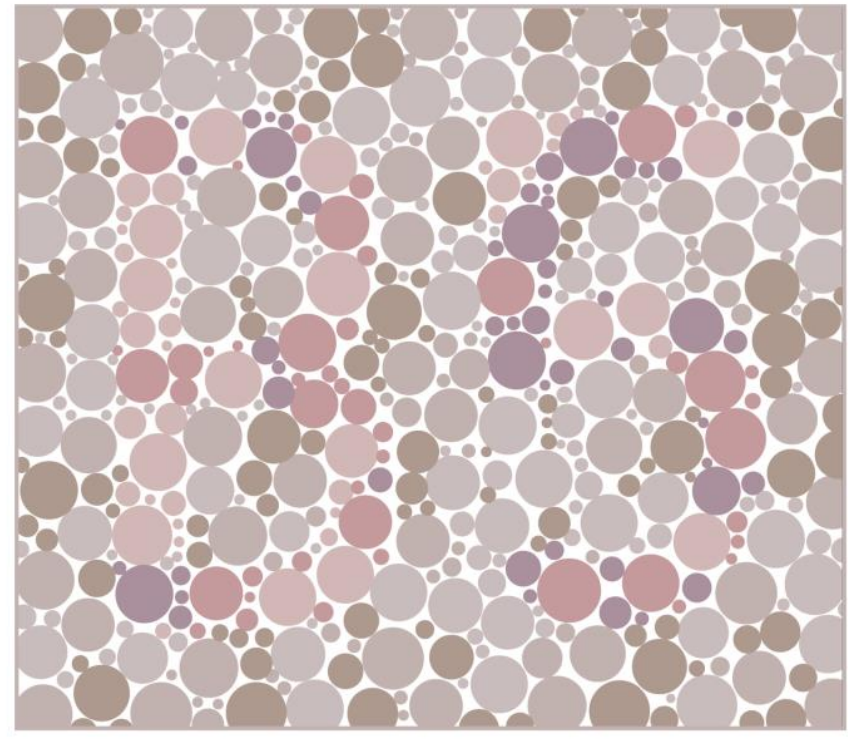

Tafel 19: Tafel mit alternativem Sehzeichen (Zeichen: B5) 


\section{Anhang 2}

iPad $\AA$ - Technische Daten(Apple, 2013b)

\section{Abmessungen und Gewicht}

Höhe:

242,8 mm

Breite:

$189,7 \mathrm{~mm}$

Tiefe:

$13,4 \mathrm{~mm}$

Gewicht:

$0,68 \mathrm{~kg}$ (Wi-Fi-Modell)

$0,73 \mathrm{~kg}$ (Wi-Fi- + 3G-Modell)

Display

9,7"-Multi-Touch-Widescreen-Display mit LED-Hintergrundbeleuchtung, Hochglanzanzeige und IPS-Technologie (24,63 cm Diagonale)

Auflösung von $1024 \times 768$ Pixeln bei 132 ppi

Fettabweisende Beschichtung

Unterstützung für die Anzeige mehrerer Sprachen und Zeichen gleichzeitig

Drahtlostechnologie und Funknetz

Wi-Fi-Modell

- Wi-Fi (802.11 a/b/g/n)

- Bluetooth $2.1+$ EDR

Wi-Fi- + 3G-Modell

- UMTS/HSDPA $(850,1900,2100 \mathrm{MHz})$

- $\operatorname{GSM} / \operatorname{EDGE}(850,900,1800,1900 \mathrm{MHz})$

- Nur Datentransfer

- Wi-Fi (802.11 a/b/g/n)

- Bluetooth $2.1+$ EDR

Ortungsfunktion

- Wi-Fi

- Digitaler Kompass

- Assisted GPS (beim Wi-Fi- + 3G-Modell)

- Funknetzunterstützung (beim Wi-Fi- + 3G-Modell)

Lieferumfang

- iPad

- Dock Connector-zu-USB-Kabel

- 10W Power Adapter (Netzteil)

- Dokumentation

Kapazität

- 16 GB, 32 GB oder 64 GB Flash-Laufwerk

Prozessor

- Speziell entwickelter, hochleistungsfähiger $1 \mathrm{GHz}$ Apple-A4-SOC-Chip (System-on-a-Chip) mit niedrigem Stromverbrauch

\section{Sensoren}


- Beschleunigungssensor

- Umgebungslichtsensor

Audiowiedergabe

- Frequenzbereich: $20 \mathrm{~Hz}$ bis $20.000 \mathrm{~Hz}$

- Unterstützte Audioformate: HE-AAC (V1), AAC (16 bis $320 \mathrm{KBit} / \mathrm{Sek}$.), Protected AAC (aus dem iTunes Store), MP3 (16 bis $320 \mathrm{KBit} / \mathrm{Sek}$.), MP3 VBR, Audible (Formate 2, 3 und 4), Apple Lossless, AIFF und WAV

- Maximale Lautstärke vom Benutzer festlegbar

TV und Video

- Unterstützung für 1024 x 768 Pixel mit dem Dock Connector-zu-VGA-Adapter; 576p und 480p mit dem Apple AV-Kabel (Komponentenvideo); 576i und 480i mit dem Apple AV-Kabel (Composite-Video)

- H.264 Video mit bis zu 720p und 30 Bildern pro Sekunde, Main Profile Level 3.1 mit AAC-LC Audio mit bis zu $160 \mathrm{KBit} / \mathrm{Sek}$., $48 \mathrm{kHz}$, Stereo-Audio in den Formaten .m4v, .mp4 und .mov; MPEG-4 Video mit bis zu 2,5 MBit/Sek., 640 x 480 Pixel, 30 Bilder pro Sekunde, Simple Profile mit AAC-LC Audio mit bis zu $160 \mathrm{KBit} / S e k ., 48 \mathrm{kHz}$, Stereo-Audio in den Formaten .m4v, .mp4 und .mov; Motion JPEG (M-JPEG) mit bis zu 35 MBit/Sek., 1280 x 720 Pixel, 30 Bilder pro Sekunde, Audio im ulawFormat, PCM-Stereo-Audio im .avi-Format

\section{Unterstützte E-Mail-Anhänge}

- Anzeigbare Dokumenttypen: .jpg, .tiff, .gif (Bilder); .doc und .docx (Microsoft Word); .htm und .html (Webseiten); .key (Keynote); .numbers (Numbers); .pages (Pages); .pdf (Vorschau und Adobe Acrobat); .ppt und .pptx (Microsoft PowerPoint); .txt (Text); .rtf (Rich Text Format); .vcf (Kontaktdaten); .xls und .xlsx (Microsoft Excel)

\section{Sprachen}

- Sprachunterstützung für Englisch, Französisch, Deutsch, Japanisch, Niederländisch, Italienisch, Spanisch, Chinesisch (vereinfacht), Russisch

- Tastaturunterstützung für Englisch (USA), Englisch (Großbritannien), Französisch (Frankreich, Kanada), Deutsch, Japanisch (QWERTY), Niederländisch, Flämisch, Spanisch, Italienisch, Chinesisch (vereinfacht Handschrift und Pinyin), Russisch

- Wörterbuchunterstützung für Englisch (USA), Englisch (Großbritannien), Französisch, Französisch (Kanada), Französisch (Schweiz), Deutsch, Japanisch, Niederländisch, Flämisch, Spanisch, Italienisch, Chinesisch (vereinfacht - Handschrift und Pinyin), Russisch

\section{Bedienungshilfen}

- Unterstützung für die Wiedergabe von Inhalten mit erweiterten Untertiteln

- VoiceOver Bildschirmlesetechnologie

- Bildschirmfüllender Zoom

- Weiß auf Schwarz

- Mono-Audioausgabe

Batterie und Stromversorgung4

- Integrierte wiederaufladbare Lithium-Polymer-Batterie (25 Wattstunden)

- Bis zu 10 Stunden Surfen im Web mit Wi-Fi, Video- oder Musikwiedergabe 
- Aufladen über das Netzteil oder einen USB-Anschluss am Computer Ein- und Ausgänge

- 30-poliger Dock-Anschluss

- Stereo-Kopfhöreranschluss (3,5 mm)

- Integrierter Lautsprecher

- Mikrofon

- Fach für Micro-SIM-Karte (nur beim Wi-Fi + 3G Modell)

Tasten und Steuerelemente am Gehäuse

- Ein/Aus, Standby

- Display-ausrichtung verriegeln

- Lautstärke

- Home

Systemvoraussetzungen für Mac

- Mac mit USB 2.0 Anschluss

- Mac OS X 10.5.8 (oder neuer)

- iTunes 9.0 oder neuer

- iTunes Store Account

- Internetzugang

Systemvoraussetzungen für Windows

- PC mit USB 2.0 Anschluss

- Windows 7, Windows Vista, Windows XP Home oder Professional mit Service Pack 3 (oder neuer)

- iTunes 9.0 oder neuer

- iTunes Store Account

- Internetzugang

Umgebungsbedingungen

- Betriebstemperatur: $0^{\circ} \mathrm{C}$ bis $35^{\circ} \mathrm{C}$

- Lagertemperatur: $-20^{\circ} \mathrm{C}$ bis $45^{\circ} \mathrm{C}$

- Relative Luftfeuchtigkeit: $5 \%$ bis $95 \%$, nicht kondensierend

iPad®2- Technische Daten (Apple, 2013a)

Modelle

$\mathrm{Wi}-\mathrm{Fi}$

$\mathrm{Wi}-\mathrm{Fi}+3 \mathrm{G}$

Abmessungen und Gewicht

Wi-Fi

- Höhe: $241,2 \mathrm{~mm}$

- Breite: $185,7 \mathrm{~mm}$

- Tiefe: $8,8 \mathrm{~mm}$

- Gewicht: $601 \mathrm{~g}$

$\mathrm{Wi}-\mathrm{Fi}+3 \mathrm{G}$

- Höhe: 241,2 mm

- Breite: 185,7 mm

- Tiefe: $8,8 \mathrm{~mm}$ 
- Gewicht: $613 \mathrm{~g}$ (Wi-Fi + 3G Modell)

Kapazität

- 16 GB

\section{Drahtlose Kommunikation und Mobilfunk}

Wi-Fi

- Wi-Fi $(802.11 \mathrm{a} / \mathrm{b} / \mathrm{g} / \mathrm{n})$

- Bluetooth $2.1+$ EDR Technologie

$\mathrm{Wi}-\mathrm{Fi}+3 \mathrm{G}$

- Wi-Fi $(802.11 \mathrm{a} / \mathrm{b} / \mathrm{g} / \mathrm{n})$

- Bluetooth 2.1 + EDR Technologie

- Wi-Fi + 3G Modell: UMTS/HSDPA/HSUPA (850, 900, 1900, $2100 \mathrm{MHz}$ ), GSM/EDGE $(850,900,1800,1900 \mathrm{MHz})$

- Nur Daten

Display

- 9,7" Multi-Touch Hochglanz-Widescreendisplay (24,63 cm Diagonale) mit LEDHintergrundbeleuchtung und IPS-Technologie

- Auflösung von 1024 x 768 Pixeln bei 132 ppi

- Fettabweisende Beschichtung

- Unterstützung für die Anzeige mehrerer Sprachen und Zeichen gleichzeitig Chip

- Dual-Core A5

Kamera, Fotos und Videoaufnahme

- Rückkamera: Videoaufnahme, HD (720p) mit bis zu 30 Bildern pro Sekunde und Audio, Fotokamera mit 5fach Digitalzoom

- Frontkamera: Videoaufnahme, VGA mit bis zu 30 Bildern pro Sekunde und Audio, Fotokamera in VGA-Qualität

- Belichtungsanpassung per Fingertipp für Videos und Fotos

- Geotagging für Fotos und Videos via Wi-Fi

Externe Tasten und Anschlüsse

Externe Tasten und Steuerelemente Anschlüsse und Ein-/Ausgänge

\section{Stromversorgung und Batterie}
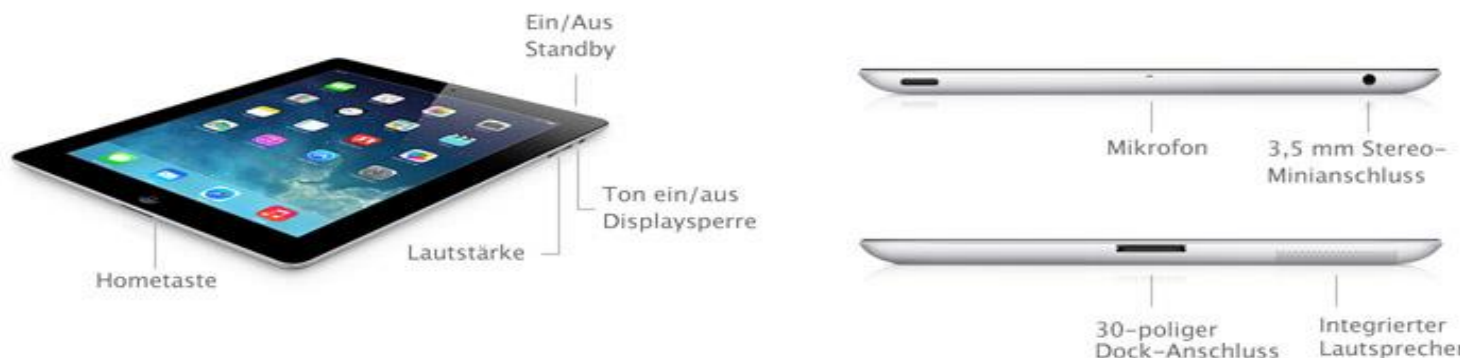

$\mathrm{Wi}-\mathrm{Fi}$

- Integrierte wiederaufladbare Lithium-Polymer-Batterie mit 25 Wattstunden

- Bis zu 10 Stunden Surfen im Web mit Wi-Fi, Video- oder Musikwiedergabe

- Aufladen über das Netzteil oder einen USB Anschluss am Computer $\mathrm{Wi}-\mathrm{Fi}+3 \mathrm{G}$

- Integrierte wiederaufladbare Lithium-Polymer-Batterie mit 25 Wattstunden 
- Bis zu 10 Stunden Surfen im Web mit Wi-Fi, Video- oder Musikwiedergabe

- Bis zu 9 Stunden Surfen im Web über ein mobiles Datennetz

- Aufladen über das Netzteil oder einen USB Anschluss am Computer

Eingabe/Ausgabe

Wi-Fi

- 30-poliger Dock-Anschluss

- 3,5 mm Stereo-Minianschluss (Kopfhörer)

- Integrierter Lautsprecher

- Mikrofon

$\mathrm{Wi}-\mathrm{Fi}+3 \mathrm{G}$

- 30-poliger Dock-Anschluss

- 3,5 mm Stereo-Minianschluss (Kopfhörer)

- Integrierter Lautsprecher

- Mikrofon

- Fach für Micro-SIM-Karte (nur beim Wi-Fi + 3G Modell)

Sensoren

- Beschleunigungssensor

- Umgebungslichtsensor

- Gyrosensor

Ortung

Wi-Fi

- Wi-Fi

- Digitaler Kompass

$\underline{\mathrm{Wi}-\mathrm{Fi}+3 \mathrm{G}}$

- Wi-Fi

- Digitaler Kompass

- Assisted GPS

- Mobilfunknetz

Audiowiedergabe

- Frequenzbereich: $20 \mathrm{~Hz}$ bis $20.000 \mathrm{~Hz}$

- Unterstützte Audioformate: HE-AAC (V1 und V2), AAC (8 bis $320 \mathrm{KBit} / \mathrm{s}$ ), Protected AAC (aus dem iTunes Store), MP3 (8 bis $320 \mathrm{KBit} / \mathrm{s}$ ), MP3 VBR, Audible (Formate 2, 3 und 4, Audible Enhanced Audio, AAX und AAX+), Apple Lossless, AIFF und WAV

- Vom Benutzer festlegbare maximale Lautstärke

- Dolby Digital 5.1 Surround-Sound Pass-Through mit Apple Digital AV Adapter (separat erhältlich)

\section{TV und Video}

- Unterstützung für Air Play Mirroring an Apple TV mit 720p

- „Bildschirme synchronisieren“ und Videoausgabe: Unterstützung für bis zu 1080p mit Apple Digital AV Adapter oder Apple VGA Adapter (Adapter separat erhältlich)

- Videoausgabe: Unterstützung für $576 p$ und $480 p$ mit Apple AV Kabel (Komponentenvideo) und für 576i und 480i mit Apple AV Kabel für Composite-Video (Kabel separat erhältlich)

- Unterstützte Videoformate: H.264 Video: bis zu 1080p, 30 Bilder pro Sekunde, 
High Profile Level 4.1 mit AAC-LC Audio bis zu $160 \mathrm{KBit} / \mathrm{s}, 48 \mathrm{kHz}$, Stereo-Audio in den Formaten .m4v, .mp4 und .mov; MPEG-4 Video: bis zu 2,5 MBit/s, 640 x 480 Pixel, 30 Bilder pro Sekunde, Simple Profile mit AAC-LC Audio bis zu $160 \mathrm{KBit} / \mathrm{s}, 48 \mathrm{kHz}$, Stereo-Audio in den Formaten . $\mathrm{m} 4 \mathrm{v}$, .mp4 und .mov; Motion JPEG (M-JPEG): bis zu $35 \mathrm{MBit} / \mathrm{s}$, $1280 \times 720$ Pixel, 30 Bilder pro Sekunde, Audio im Format ulaw, PCM Stereo-Audio im Format .avi

\section{Unterstützte E-Mail Anhänge}

Anzeigbare Dokumenttypen: .jpg, .tiff und .gif (Bilder), .doc und .docx (Microsoft Word), .htm und .html (Webseiten), .key (Keynote), .numbers (Numbers), .pages (Pages), .pdf (Vorschau und Adobe Acrobat), .ppt und .pptx (Microsoft PowerPoint), .txt (Text), .rtf (Rich Text Format), .vcf (Kontaktdaten), .xls und .xlsx (Microsoft Excel)

\section{Sprachen}

- Sprachunterstützung: Englisch (USA), Englisch (Großbritannien), Chinesisch (vereinfacht), Chinesisch (traditionell), Französisch, Deutsch, Italienisch, Japanisch, Koreanisch, Spanisch, Arabisch, Katalanisch, Kroatisch, Tschechisch, Dänisch, Niederländisch, Finnisch, Griechisch, Hebräisch, Ungarisch, Indonesisch, Malaiisch, Norwegisch, Polnisch, Portugiesisch (Portugal), Portugiesisch (Brasilien), Rumänisch, Russisch, Slowakisch, Schwedisch, Thailändisch, Türkisch, Ukrainisch, Vietnamesisch

- Tastaturunterstützung für Englisch (USA), Englisch (Großbritannien), Chinesisch - vereinfacht (Handschrift, Pinyin, Stroke), Chinesisch traditionell (Handschrift, Pinyin, Zhuyin, Cangjie, Stroke), Französisch (Frankreich), Französisch (Kanada), Französisch (Schweiz), Deutsch (Deutschland), Deutsch (Schweiz), Italienisch, Japanisch (Romaji, Kana), Koreanisch, Spanisch, Arabisch, Bulgarisch, Katalanisch, Cherokee, Kroatisch, Tschechisch, Dänisch, Niederländisch, Emoji, Estnisch, Finnisch, Flämisch, Griechisch, Hawaiisch, Hebräisch, Hindi, Ungarisch, Isländisch, Indonesisch, Lettisch, Litauisch, Mazedonisch, Malaiisch, Norwegisch, Polnisch, Portugiesisch (Portugal), Portugiesisch (Brasilien), Rumänisch, Russisch, Serbisch (Kyrillisch/Lateinisch), Slowakisch, Schwedisch, Thailändisch, Tibetisch, Türkisch, Ukrainisch, Vietnamesisch

- Wörterbuchunterstützung (aktiviert Textvorhersage und automatische Korrektur) für Englisch (USA), Englisch (Großbritannien), Chinesisch (vereinfacht), Chinesisch (traditionell), Französisch (Frankreich), Französisch (Kanada), Französisch (Schweiz), Deutsch, Italienisch, Japanisch (Romaji, Kana), Koreanisch, Spanisch, Arabisch, Katalanisch, Cherokee, Kroatisch, Tschechisch, Dänisch, Niederländisch, Estnisch, Finnisch, Flämisch, Griechisch, Hawaiisch, Hebräisch, Hindi, Ungarisch, Indonesisch, Lettisch, Litauisch, Malaiisch, Norwegisch, Polnisch, Portugiesisch (Portugal), Portugiesisch (Brasilien), Rumänisch, Russisch, Slowakisch, Schwedisch, Thailändisch, Türkisch, Ukrainisch, Vietnamesisch

\section{Bedienungshilfen}

- VoiceOver Bildschirmlesetechnologie 
- Unterstützung für die Wiedergabe von Inhalten mit erweiterten Untertiteln

- AssistiveTouch Oberfläche für adaptives Zubehör

- Bildschirmfüllender Zoom

- Große Schriften

- Anzeigeoption Weiß-auf-Schwarz

- Lautstärkeanpassung links/rechts

Umgebungsbedingungen

- Betriebsumgebungstemperatur: $0^{\circ} \mathrm{C}$ bis $35^{\circ} \mathrm{C}$

- Lagertemperatur: $-20^{\circ} \mathrm{C}$ bis $45^{\circ} \mathrm{C}$

- Relative Luftfeuchtigkeit: $5 \%$ bis $95 \%$, nicht kondensierend Systemvoraussetzungen

- Apple ID (für einige Funktionen erforderlich)

- Internetzugang5

- Synchronisierung mit iTunes auf einem Mac oder PC erfordert:

- Mac: OS X 10.5.8 oder neuer

- PC: Windows 7, Windows Vista oder Windows XP Home oder Professional mit Service Pack 3 oder neuer

- $\quad$ iTunes 10.5 oder neuer

\section{Lieferumfang}

- iPad

- Dock Connector auf USB Kabel

- 10W USB Power Adapter (Netzteil)

Integrierte Apps

- Safari

- Fotos

- App Store

- Karten

- Photo Booth

- Erinnerungen

- Kamera

- Mail

- FaceTime

- iTunes

- Musik

- Uhr

- Kalender

- Nachrichten

- Zeitungskiosk

- Videos

- Game Center

- Kontakte

- Notizen 


\section{Anhang 3}

\section{Berechnung des Cohen's-Kappa}

Tab. 1: Berechnung Kappa - alle Nutzer

\begin{tabular}{|c|c|c|}
\hline & Kappa & $p$-Wert \\
\hline 2 & 0.774 & $<0.001$ \\
\hline 3 & 0.880 & $<0.001$ \\
\hline 4 & 0.497 & $<0.001$ \\
\hline 5 & 0.975 & $<0.001$ \\
\hline 6 & 0.665 & $<0.001$ \\
\hline 7 & 0.924 & $<0.001$ \\
\hline 8 & 0.815 & $<0.001$ \\
\hline 9 & 0.671 & $<0.001$ \\
\hline 10 & 0.895 & $<0.001$ \\
\hline Tafel & & \\
\hline 11 & 0.880 & $<0.001$ \\
\hline 12 & 0.449 & $<0.001$ \\
\hline 13 & 0.783 & $<0.001$ \\
\hline 14 & 1.000 & $<0.001$ \\
\hline 15 & 0.412 & $<0.001$ \\
\hline 16 & 0.667 & $<0.001$ \\
\hline 17 & 0.868 & $<0.001$ \\
\hline 18 & 0.740 & $<0.001$ \\
\hline 19 & 0.542 & $<0.001$ \\
\hline
\end{tabular}


Tab. 2: Berechnung Kappa - Farbfehlsichtige, n.b.: nicht berechenbar

\begin{tabular}{|c|c|c|}
\hline & Kappa & $p$-Wert \\
\hline 2 & 1.000 & $<0.001$ \\
\hline 3 & 0.824 & $<0.001$ \\
\hline 4 & 0.355 & 0.106 \\
\hline 5 & 1.000 & $<0.001$ \\
\hline 6 & 0.580 & 0.001 \\
\hline 7 & 0.735 & $<0.001$ \\
\hline 8 & 0.762 & 0.005 \\
\hline 9 & 0.110 & 0.327 \\
\hline 10 & 0.725 & $<0.001$ \\
\hline Tafel & & \\
\hline 11 & 0.444 & 0.025 \\
\hline 12 & 0.486 & 0.001 \\
\hline 13 & 0.667 & $<0.001$ \\
\hline 14 & 1.000 & $<0.001$ \\
\hline 15 & n.b. & \\
\hline 16 & n.b. & \\
\hline 17 & 0.769 & $<0.001$ \\
\hline 18 & 0.390 & 0.024 \\
\hline 19 & 0.674 & $<0.001$ \\
\hline
\end{tabular}


Tab.3: Berechnung Kappa - Farbnormale, n.b.: nicht berechenbar

\begin{tabular}{|c|c|c|}
\hline & Kappa & $p$-Wert \\
\hline 2 & 0.703 & $<0.001$ \\
\hline 3 & n.b. & \\
\hline 4 & 0.431 & $<0.001$ \\
\hline 5 & 0.955 & $<0.001$ \\
\hline 6 & 0.533 & $<0.001$ \\
\hline 7 & 0.955 & $<0.001$ \\
\hline 8 & n.b. & \\
\hline 9 & 0.699 & 0.327 \\
\hline 10 & n.b. & \\
\hline Tafel & & \\
\hline 11 & 0.871 & $<0.001$ \\
\hline 12 & 0.321 & 0.001 \\
\hline 13 & n.b. & \\
\hline 14 & n.b. & \\
\hline 15 & 0.408 & $<0.001$ \\
\hline 16 & 0.073 & 0.324 \\
\hline 17 & 0.661 & 0.001 \\
\hline 18 & 0.659 & $<0.001$ \\
\hline 19 & 0.451 & $<0.001$ \\
\hline
\end{tabular}


Tab. 4: Berechnung Kappa - Buch vs. iPad1®, n.b.: nicht berechenbar

\begin{tabular}{|c|c|c|}
\hline & Kappa & $p$-Wert \\
\hline 2 & 0.848 & $<0.001$ \\
\hline 3 & 0.474 & 0.002 \\
\hline 4 & 0.302 & 0.005 \\
\hline 5 & 1.000 & $<0.001$ \\
\hline 6 & 0.714 & $<0.001$ \\
\hline 7 & 1.000 & $<0.001$ \\
\hline 8 & 0.630 & 0.005 \\
\hline 9 & 1.000 & $<0.001$ \\
\hline 10 & 1.000 & $<0.001$ \\
\hline Tafel & & \\
\hline 11 & 0.825 & 0.001 \\
\hline 12 & 0.474 & 0.001 \\
\hline 13 & n.b. & \\
\hline 14 & n.b. & \\
\hline 15 & 1.000 & 0.002 \\
\hline 16 & 0.630 & 0.005 \\
\hline 17 & 1.000 & 0.002 \\
\hline 18 & 1.000 & 0.002 \\
\hline 19 & 0.512 & $<0.001$ \\
\hline
\end{tabular}


Tab.5: Berechnung Kappa-iPad1 $® v$ s. iPad2®, n.b.: nicht berechenbar

\begin{tabular}{|c|c|c|}
\hline & Карра & $p-$ Wer \\
\hline 2 & 0.091 & 0.311 \\
\hline 3 & n.b. & \\
\hline 4 & 0.333 & 0.001 \\
\hline 5 & 0.167 & 0.035 \\
\hline 6 & 1.000 & $<0.001$ \\
\hline 7 & 0.167 & 0.035 \\
\hline 8 & 0.630 & 0.005 \\
\hline 9 & 0.167 & 0.035 \\
\hline 10 & 1.000 & $<0.001$ \\
\hline Tafel & & \\
\hline 11 & 0.167 & 0.035 \\
\hline 12 & n.b. & \\
\hline 13 & n.b. & \\
\hline 14 & n.b. & \\
\hline 15 & 1.000 & $<0.001$ \\
\hline 16 & 0.630 & 0.005 \\
\hline 17 & 0.630 & 0.005 \\
\hline 18 & 1.000 & $<0.001$ \\
\hline 19 & 0.383 & 0.008 \\
\hline
\end{tabular}

